\title{
Cascades and Dissipative Anomalies in Compressible Fluid Turbulence
}

\author{
Gregory L. Eyink ${ }^{1,2, *}$ and Theodore D. Drivas ${ }^{1}$ \\ ${ }^{1}$ Department of Applied Mathematics \& Statistics, The Johns Hopkins University, \\ Baltimore, Maryland 21218-2682, USA \\ ${ }^{2}$ Department of Physics \& Astronomy, The Johns Hopkins University, \\ Baltimore, Maryland 21218-2682, USA
}

(Received 11 April 2017; revised manuscript received 5 June 2017; published 12 February 2018)

\begin{abstract}
We investigate dissipative anomalies in a turbulent fluid governed by the compressible Navier-Stokes equation. We follow an exact approach pioneered by Onsager, which we explain as a nonperturbative application of the principle of renormalization-group invariance. In the limit of high Reynolds and Péclet numbers, the flow realizations are found to be described as distributional or "coarse-grained" solutions of the compressible Euler equations, with standard conservation laws broken by turbulent anomalies. The anomalous dissipation of kinetic energy is shown to be due not only to local cascade but also to a distinct mechanism called pressure-work defect. Irreversible heating in stationary, planar shocks with an ideal-gas equation of state exemplifies the second mechanism. Entropy conservation anomalies are also found to occur via two mechanisms: an anomalous input of negative entropy (negentropy) by pressure work and a cascade of negentropy to small scales. We derive "4/5th-law"-type expressions for the anomalies, which allow us to characterize the singularities (structure-function scaling exponents) required to sustain the cascades. We compare our approach with alternative theories and empirical evidence. It is argued that the "Big Power Law in the Sky" observed in electron density scintillations in the interstellar medium is a manifestation of a forward negentropy cascade or an inverse cascade of usual thermodynamic entropy.
\end{abstract}

DOI: 10.1103/PhysRevX.8.011022

\section{INTRODUCTION}

Compressible fluids play a vital role in problems of astrophysics (interstellar medium or ISM [1], star formation [2,3]), applied physics (inertial confinement fusion [4]), and engineering (high-temperature reactive flows [5], supersonic aircraft design [6]). Relativistic fluids are necessarily compressible, of course, and occur in astrophysical flows (pulsars [7], gamma-ray bursts [8]), high-energy physics (heavy-ion collisions [9]), and condensed matter physics (graphene [10-12], strange metals $[13,14])$. In many of the above examples, the fluid is either directly observed or indirectly inferred to be in a turbulent state. The nature of compressible turbulence has been highly controversial, however. It is currently debated whether the notion of an "energy cascade" (as it was developed by Kolmogorov [15-17], Obukhov [18], Onsager [19,20], Heisenberg [21], and von Weizsäcker [22] to describe incompressible fluid turbulence) is applicable at all to turbulence in compressible fluids. On the one hand, some authors argue that, much the

\footnotetext{
*eyink@jhu.edu
}

Published by the American Physical Society under the terms of the Creative Commons Attribution 4.0 International license. Further distribution of this work must maintain attribution to the author(s) and the published article's title, journal citation, and DOI.
Subject Areas: Astrophysics, Fluid Dynamics, Plasma Physics same as for incompressible turbulence, compressible fluids possess a turbulent inertial range "which is immune from direct effects of viscosity and large-scale forcing" [23] through which kinetic energy is transferred to small scales by a cascade process that is local in scale. On the other hand, exact statistical relations have been derived for nonrelativistic compressible turbulence [24] and for relativistic turbulence [25], which do not involve kinetic energy and which have been invoked to argue that "the interpretation of the Kolmogorov relation for the incompressible turbulence in terms of the energy cascade may be misleading" [25]. This is a controversy whose resolution has profound consequences for all physical systems where compressible fluid turbulence manifests itself.

The primary physical effects of cascades in incompressible fluids are "dissipative anomalies," in which ideal invariants of the fluid equations, such as kinetic energy, are nonconserved even in the inviscid or high Reynoldsnumber limit. This effect was deduced semiphenomenologically from geophysical observations by Taylor [26] and confirmed in classical wind-tunnel experiments [27]. For modern evidence from numerical simulations and experiments, see Refs. [28-30]. This type of empirical evidence motivated the theories of Kolmogorov [15-17], Obukhov [18], Onsager [19,20], Heisenberg [21], and von Weizsäcker [22]. A particularly deep contribution was made by Onsager $[20,31]$, who argued that turbulent fluids could be described 
by singular (weak) solutions of incompressible Euler equations whose kinetic-energy balance equations would be afflicted with an anomaly due to the nonlinear cascade mechanism [32]. Onsager's derivation was made using a (smoothed) version of a point-splitting regularization, which yielded for the anomaly an expression closely related to the Kolmogorov 4/5th law but valid for individual flow realizations, without averaging over ensembles [31]. Kolmogorov's weaker statistical relation is, of course, well known to physicists; e.g., Polyakov has pointed out the formal analogy of Kolmogorov's relation and its pointsplitting derivation to axial anomalies in quantum gauge field theories [33,34]. Onsager's deeper contribution has received little attention in the physics community, on the other hand, although the many predictions of Onsager's analysis are consistent with all available experimental evidence. In particular, his prediction of $1 / 3$ Hölder singularities for the velocity field has been confirmed experimentally (e.g., Ref. [35]). In fact, an entire multifractal spectrum of singularities has been measured, as in the later elaboration of Parisi-Frisch [36,37].

We show here that the Onsager theory carries over to compressible fluids, completing earlier work of Aluie $[23,38]$. There have been extensive further developments of Onsager's ideas, which we shall exploit. In particular, we closely follow the approaches of Eyink [39,40], Constantin et al. [41], and Duchon-Robert [42], who derived necessary conditions for dissipative anomalies of kinetic energy in turbulent solutions of incompressible Euler equations. Subsequently, there has been deep mathematical work constructing dissipative, Hölder-continuous Euler solutions for the incompressible case by "convex integration" methods, using ideas originating in the Nash-Kuiper theorem and Gromov's h-principle (e.g., see DeLellis and Szkeleyhidi $[43,44])$. This circle of ideas recently led to a proof that Onsager's 1/3 Hölder exponent is sharp [45]. These remarks might suggest that a high level of mathematical sophistication is necessary to grasp the essentials of Onsager's ideas on turbulent weak solutions. This is not the case. As a matter of fact, Onsager's ideas are closely related to standard physical notions of spatial coarse graining and renormalization-group invariance [46-48]. See especially Ref. [49], Sec. IV. In addition to extending Onsager's approach to compressible fluids and deriving new testable predictions, we also carefully explain the connection to renormalization-group ideas. By means of this intuitive but rigorous approach, we resolve the controversies concerning nonrelativistic compressible fluid turbulence. A comparison is made with alternative theoretical analyses, including classical ideas [50-53], and more recent works [24,54,55]; see Sec. VII. In a companion paper, we further extend our analysis to relativistic fluid turbulence [56].

Turbulence is an essentially strong-coupling problem to which perturbation theory does not apply, so that, as in
Onsager's original work, some mathematical tools of nonlinear analysis must be employed. The required background for full understanding of the finer points is mathematical analysis at a theoretical-physics level such as in Ref. [57], particularly standard spatial $L^{p}$ norms (Sec. I.D.10) and basic theory of distributions or generalized functions (Sec. VI. A-B). The tools employed are similar to those in the mathematical theory of fluid shock solutions. Most of our analysis can be grasped even without that technical background but assuming just some familiarity with spatial coarse graining and fluid turbulence.

\section{COMPRESSIBLE NAVIER-STOKES AND HYDRODYNAMIC SCALING}

The model equations that we employ for (nonrelativistic) compressible fluids in this paper are the standard NavierStokes equations in space dimension $d$. These govern the evolution of the conserved densities (per volume) of mass $\rho$, momentum $\mathbf{j}$, and total energy $E=|\mathbf{j}|^{2} / 2 \rho+u$ (kineticenergy density $|\mathbf{j}|^{2} / 2 \rho$ plus internal-energy density $u$ ) by

$$
\begin{gathered}
\partial_{t} \rho+\boldsymbol{\nabla} \cdot(\rho \mathbf{v})=0 \\
\partial_{t}(\rho \mathbf{v})+\boldsymbol{\nabla} \cdot(\rho \mathbf{v} \mathbf{v}+p \mathbf{I}-2 \eta \mathbf{S}-\zeta \Theta \mathbf{I})=0, \\
\partial_{t}\left(\frac{1}{2} \rho v^{2}+u\right)+\boldsymbol{\nabla} \cdot\left[\left(u+p+\frac{1}{2} \rho v^{2}\right) \mathbf{v}\right. \\
-\kappa \boldsymbol{\nabla} T-2 \eta \mathbf{S} \cdot \mathbf{v}-\zeta \Theta \mathbf{v}]=0 .
\end{gathered}
$$

A fluid velocity $\mathbf{v}$ has been defined conventionally by $\mathbf{v}=\mathbf{j} / \rho$, which is thus associated with the transport of mass. This is not the only possible choice of a fluid velocity (see, e.g., Refs. [58,59]), but it is the most familiar one generally employed for a nonrelativistic fluid. In Eqs. (2) and (3) above, $\eta(u, \rho)$ is the shear viscosity, $\zeta(u, \rho)$ is the bulk viscosity, $\kappa(u, \rho)$ is the thermal conductivity, and

$S_{i j}=\frac{1}{2}\left(\frac{\partial v_{i}}{\partial x_{j}}+\frac{\partial v_{i}}{\partial x_{j}}-\frac{1}{d}(\boldsymbol{\nabla} \cdot \mathbf{v}) \delta_{i j}\right), \quad \Theta=\boldsymbol{\nabla} \cdot \mathbf{v}$

are the strain tensor and dilatational field, respectively. Here, we admit any thermodynamically consistent relations for the pressure $p(u, \rho)$ (the equation of state) and for the absolute temperature $T(u, \rho)$.

It should be pointed out that this set of equations has some well-known deficiencies in representing the internal structure of strong shocks, whose thickness is of the order of the mean-free-path length of the fluid [60-62]. This may cause concern since compressible fluid turbulence is well known to develop numerous small-scale "shocklets." A more fundamental model for the dynamics of a neutral (nonionized) gas would be the Boltzmann kinetic equation, whose solutions agree well with the experimental data 
for strong shocks. However, we expect that all of our conclusions below will still apply if such a kinetic description is employed, as we are concerned with length scales much greater than the width of the shock front where, for both kinetic and fluid models, a similar description emerges as a discontinuous weak or distributional solution of the compressible Euler equations [63]. The use of a fluid description from the outset greatly simplifies our analysis, but similar arguments should carry over to kinetic theory. We do not discuss the effects of molecular noise either, which, for a thermodynamically consistent description of a compressible Navier-Stokes fluid, requires stochastic PDEs with suitable multiplicative noise given by a fluctuation-dissipation relation [64,65]. The effects of such noise are quite significant, presumably leading to a "stochastic anomaly" in addition to the dissipative anomaly already discussed [66-69]. This is an issue of fundamental importance for the problems of predicting, reproducing, or controlling turbulent flows [70,71], but, as argued further below, the addition of thermal noise does not alter our conclusions in this paper on dissipative anomalies.

Compressible fluid turbulence is characterized by several dimensionless number groups, which are revealed by a scaling of the fluid equations. There is more than one way to rescale the equations. Here, we follow a simple approach, introducing dimensionless variables

$$
\begin{gathered}
\hat{\rho}=\rho / \rho_{0}, \quad \hat{\mathbf{v}}=\mathbf{v} / v_{0}, \quad \hat{u}=u / \rho_{0} v_{0}^{2}, \\
\hat{\mathbf{x}}=\mathbf{x} / L_{0}, \quad \hat{t}=t /\left(L_{0} / v_{0}\right), \\
\hat{p}=p / \rho_{0} v_{0}^{2}, \quad \hat{T}=T / T_{0} .
\end{gathered}
$$

In the equations above, $\rho_{0}$ and $v_{0}$ are typical densities and velocities, such as spatial mean or r.m.s. values. Here, $L_{0}$ is the large length scale of the turbulent flow, e.g., the integral length, or another length characterizing the scale of injection of kinetic energy either by external stirring or by initial data. For the temperature scale $T_{0}$, we may also take a r.m.s. value or, alternatively, $T_{0}=T\left(\rho_{0} v_{0}^{2}, \rho_{0}\right)$. The nondimensonalized equations of motion then become

$$
\begin{gathered}
\partial_{\hat{t}} \hat{\rho}+\hat{\boldsymbol{\nabla}} \cdot(\hat{\rho} \hat{\mathbf{v}})=0, \\
\partial_{\hat{t}}(\hat{\rho} \hat{\mathbf{v}})+\hat{\boldsymbol{\nabla}} \cdot(\hat{\rho} \hat{\mathbf{v}} \hat{\mathbf{v}}+\hat{p} \mathbf{I}-2 \hat{\eta} \hat{\mathbf{S}}-\hat{\zeta} \hat{\Theta} \mathbf{I})=0, \\
\partial_{\hat{t}}\left(\frac{1}{2} \hat{\rho} \hat{v}^{2}+\hat{u}\right)+\hat{\boldsymbol{\nabla}} \cdot\left[\left(\hat{u}+\hat{p}+\frac{1}{2} \hat{\rho} \hat{v}^{2}\right) \hat{\mathbf{v}}\right. \\
-\hat{\kappa} \hat{\boldsymbol{\nabla}} \hat{T}-2 \hat{\eta} \hat{\mathbf{S}} \cdot \hat{\mathbf{v}}-\hat{\zeta} \hat{\Theta} \hat{\mathbf{v}}]=0,
\end{gathered}
$$

where

$$
\hat{\eta}(\hat{u}, \hat{\rho})=\frac{\eta}{\rho_{0} v_{0} L_{0}}, \quad \hat{\zeta}(\hat{u}, \hat{\rho})=\frac{\zeta}{\rho_{0} v_{0} L_{0}}
$$

are inverse Reynolds numbers associated with the shear and bulk viscosities, respectively, and

$$
\hat{\kappa}(\hat{u}, \hat{\rho})=\frac{\kappa T_{0}}{\rho_{0} v_{0}^{3} L_{0}}
$$

is an inverse Péclet number. Fully developed turbulent flow occurs when the Reynolds and Péclet numbers (as functions of $\hat{u}, \hat{\rho}$ ) are uniformly very large compared to unity, and $\hat{\eta}, \hat{\zeta}, \hat{\kappa}$ are small. It should be kept in mind that these dimensionless transport quantities are generally made smaller not by decreasing $\eta, \zeta, \kappa$ but instead typically by increasing $\rho_{0}, v_{0}$, or especially $L_{0}$. We hereafter omit the hats ".." on all variables but always assume that nondimensionalization has been carried out. In particular, when we discuss the ideal limit $\eta, \zeta, \kappa \rightarrow 0$ below, we always mean, more literally, that $\hat{\eta}, \hat{\zeta}, \hat{\kappa} \ll 1$.

There is one other dimensionless variable that plays an important role in compressible turbulence, which does not appear explicitly above. This is the Mach number or the inverse of the dimensionless sound speed:

$$
\mathrm{Ma}=1 / \hat{c}_{s}=v_{0} / c_{s},
$$

with $c_{s}=\sqrt{(\partial p / \partial \rho)_{s_{n}}}$ the adiabatic sound speed (the density derivative being taken at fixed entropy per particle $\left.s_{n}\right)$. Of course, the Mach number as defined above is also a variable function of $\hat{u}, \hat{\rho}$. The properties of compressible turbulence are strongly dependent upon the Mach number. However, the main results of the present work are valid for any Mach number. We comment below on those parts of our analysis that make any assumptions depending on the Mach number, either explicitly or implicitly.

\section{DISSIPATIVE ANOMALIES}

Immediate consequences of the compressible NavierStokes equations (1)-(3) are the kinetic-energy balance

$$
\begin{aligned}
& \partial_{t}\left(\frac{1}{2} \rho v^{2}\right)+\boldsymbol{\nabla} \cdot\left[\left(p+\frac{1}{2} \rho v^{2}\right) \mathbf{v}-2 \eta \mathbf{S} \cdot \mathbf{v}-\zeta \Theta \mathbf{v}\right] \\
& \quad=p(\boldsymbol{\nabla} \cdot \mathbf{v})-2 \eta|\mathbf{S}|^{2}-\zeta \Theta^{2}
\end{aligned}
$$

and the internal-energy balance

$\partial_{t} u+\boldsymbol{\nabla} \cdot[u \mathbf{v}-\kappa \boldsymbol{\nabla} T]=-p(\boldsymbol{\nabla} \cdot \mathbf{v})+2 \eta|\mathbf{S}|^{2}+\zeta \Theta^{2}$,

where $2 \eta|\mathbf{S}|^{2}, \zeta \Theta^{2}$ are the energy dissipation per volume due to shear and bulk viscosity, respectively. Because total energy is conserved, the kinetic energy lost is precisely equal to the internal energy gained. 
The balance equations analogous to Eqs. (14) and (15) above hold also in the limit Ma $\ll 1$ of low Mach numbers. The incompressible Navier-Stokes equation then governs the velocity field, in which only the shear viscosity survives. The temperature field obeys a passive advection equation with viscous heating as a source term. The remarkable empirical fact for 3D incompressible turbulence is that the viscous dissipation per mass $Q_{\nu}=2 \nu|\mathbf{S}|^{2}$, where $\nu=\eta / \rho$ is the kinematic viscosity, appears to have a nonvanishing limit as $\nu \rightarrow 0$. Full documentation of the relevant laboratory experiments and numerical simulations can be found in Refs. [28-30]. A more precise statement is that a distributional limit [72] of the viscous dissipation field appears to exist,

$$
Q=\mathcal{D}-\lim _{\nu \rightarrow 0} Q_{\nu}
$$

and, by experimental evidence, yields a positive measure $Q$ with multifractal scaling properties $[73,74]$ in the infinite Reynolds-number limit. As shown in the work of Duchon and Robert [42], this same measure appears as an anomaly term in the kinetic-energy balance equation

$$
\partial_{t}\left(\frac{1}{2} v^{2}\right)+\boldsymbol{\nabla} \cdot\left[\left(p+\frac{1}{2} v^{2}\right) \mathbf{v}\right]=-Q
$$

for weak solutions of the incompressible Euler equations, which are obtained by strong $L^{3}$ limits of incompressible Navier-Stokes solutions as $\nu \rightarrow 0$. Duchon and Robert [42] also derived an inertial-range expression for $Q$ closely related to the Kolmogorov 4/5th law. This allowed them to prove a refined version of the Onsager singularity theorem, namely, that $p$ th-order scaling exponents $\zeta_{p}^{v}$ of (absolute) velocity increments must satisfy $\zeta_{p}^{v} \leq p / 3$ for $p \geq 3$, or otherwise $Q \equiv 0$. See also Refs. [40,41]. The empirical fact that kinetic-energy dissipation has a nonvanishing limit for infinite Reynolds numbers, within the accuracy of current measurements, is so central to the modern understanding of incompressible fluid turbulence that it is sometimes called the "zeroth law of turbulence."

The fundamental hypothesis of the present work is that there is similarly a nonzero distributional limit

$$
Q=\mathcal{D}-\lim _{\eta, \zeta, \kappa \rightarrow 0}\left[2 \eta|\mathbf{S}|^{2}+\zeta \Theta^{2}\right]=Q_{\eta}+Q_{\zeta}>0
$$

for viscous dissipation in compressible fluid turbulence. A number of previous works have investigated the statistical properties of the viscous dissipation in compressible turbulence, e.g., Refs. [75-77]. In particular, Ref. [77] presents direct empirical evidence for the zeroth law of compressible turbulence. Furthermore, there are simple shock solutions of the compressible Navier-Stokes solution that converge as $\eta, \zeta, \kappa \rightarrow 0$ strongly (and thus distributionally) to weak solutions of compressible Euler equations for which $Q>0$. For example, see Appendix A. In this respect, the theory of compressible fluids is better off than the incompressible theory, where there are still no rigorous examples of dissipative Euler solutions obtained by the physical limit of vanishing viscosity. The purpose of the present paper is to develop the consequences of hypothesis (18) for compressible turbulence.

Furthermore, in this work we also consider the balance equation for the entropy density per volume, which is implied by the compressible Navier-Stokes equation. As is well known (e.g. Ref. [78], Chap. XII, Sec. 1; Ref. [79], Sec. 49), this balance equation has the form

$$
\begin{aligned}
\partial_{t} s & +\boldsymbol{\nabla} \cdot\left(s \mathbf{v}-\frac{\kappa \nabla T}{T}\right) \\
& =\frac{\kappa|\boldsymbol{\nabla} T|^{2}}{T^{2}}+\frac{2 \eta|\mathbf{S}|^{2}}{T}+\frac{\zeta \Theta^{2}}{T},
\end{aligned}
$$

where entropy production on the right-hand side is positive, consistent with the second law of thermodynamics. Although fluid turbulence is a strongly dissipative macroscopic process, there seem to have been remarkably few attempts to understand its consistency with the thermodynamic second law. A pioneering work in this direction is a 1949 paper of Obukhov that considered the entropy balance for incompressible fluid turbulence in the low Machnumber limit [80]. When $M a \rightarrow 0$, the compressible Navier-Stokes system (1)-(3) reduces to the divergencefree condition on velocity, $\boldsymbol{\nabla} \cdot \mathbf{v}=0$, together with the space-time constancy of density $\rho$, the incompressible Navier-Stokes equation for velocity $\mathbf{v}$,

$$
\rho\left(\partial_{t}+\mathbf{v} \cdot \boldsymbol{\nabla}\right) \mathbf{v}=-\boldsymbol{\nabla} p+\eta \Delta \mathbf{v},
$$

and the temperature equation

$$
\rho c_{P}\left(\partial_{t}+\mathbf{v} \cdot \boldsymbol{\nabla}\right) T=\boldsymbol{\nabla} \cdot(\kappa \boldsymbol{\nabla} T)+2 \eta|\mathbf{S}|^{2} .
$$

These two equations combine to give the total energy equation (3) with internal-energy density $u=\rho c_{P} T$. Here, $c_{P}$ is the specific heat (per mass) at constant pressure. See Ref. [79], Secs. 10 and 50; Ref. [81], Eqs. (5.9) and (5.10); and, for a more mathematical discussion of the low Machnumber limit, see Ref. [82], pp. 11-13. A systematic asymptotic study [83] derives the incompressible system (20) and (21) in the limit Ma $\rightarrow 0$ under the assumption that mechanical and thermal forcing are balanced and that thermal expansion effects are negligible [84], but other limits are possible. For example, if thermal forcing instead dominates mechanical forcing, then Ref. [83] found that the final viscous heating term in Eq. (21) is negligible compared with thermal diffusion, and dropping the viscous contribution yields the temperature equation considered by Obukhov [80]. All of these various low Mach-number limits are special cases of the compressible fluid equations (1)-(3) that are analyzed in this work, and all of the 
results derived here apply immediately to the incompressible fluid. The entropy analyzed by Obukhov [80] for incompressible fluids is $s=\rho c_{P} \ln T$, which is an invariant for smooth solutions of the system (20) and (21), with $\eta=\kappa=0$. Extending Obukhov's theory to compressible fluid turbulence at arbitrary Mach numbers is one of the principal motivations of our paper. In fact, our analysis will yield results that generalize those in Ref. [80] even for incompressible fluids.

While entropy is conserved for smooth solutions of the compressible Euler equations, it is natural to hypothesize that the entropy balance will also be anomalous for compressible turbulent flow and that there will be a nonvanishing limiting measure

$$
\begin{aligned}
\Sigma & =\mathcal{D}-\lim _{\eta, \zeta, \kappa \rightarrow 0}\left[\frac{\kappa|\boldsymbol{\nabla} T|^{2}}{T^{2}}+\frac{2 \eta|\mathbf{S}|^{2}}{T}+\frac{\zeta \Theta^{2}}{T}\right] \\
& =\Sigma_{\text {therm }}+\Sigma_{\text {shear }}+\Sigma_{\text {bulk }}>0
\end{aligned}
$$

describing anomalous entropy production. Simple shock solutions provide examples of such entropy anomalies for weak solutions of compressible Euler equations with step discontinuities (Appendix A), but milder Hölder singularities typical of turbulent flow should suffice for anomalous entropy production. In this work, we derive an inertialrange expression for $\Sigma$, which shows that an "inverse cascade" of entropy can provide a mechanism for an entropy anomaly, and we characterize the type of Hölder singularities of the turbulent solutions required to sustain a nonvanishing entropy flux.

\section{THEORETICAL APPROACH}

It is often assumed reflexively that fluid turbulence must be treated probabilistically. For some problems, statistical ensembles are essential, for example, for predicting the future of a given turbulent flow [66,68-70]. For many problems, however, statistical methods are wholly inadequate because one always observes in nature a single turbulent flow realization. If one wants to understand the effects of turbulence in a specific solar flare event, e.g., one does not have the luxury of averaging over an ensemble of flares. An approach that is capable of treating individual flow realizations is intrinsically more fundamental than a probabilistic treatment because statistical relations can always be obtained by subsequent averaging over ensembles. For these reasons, we make no use of statistical ensembles in the present paper. When we have reason to consider long-time steady states, we employ time averages and global space averages, denoted by $\langle\cdot\rangle$, which may be operationally obtained, in principle, from a single flow realization.

Our analysis will be based not on ensemble averaging but instead on spatial and/or temporal coarse graining, which we employ as a regularizer. Note that the existence of nonvanishing dissipative anomalies as in Eqs. (18) and (22) requires that gradients of fluid variables must diverge, $|\nabla \mathbf{v}|$, $|\nabla T| \rightarrow \infty$ as $\eta, \zeta, \kappa \rightarrow 0$. This is an ultraviolet divergence due to the development of high-wave-number excitations in the ideal limit or, as described by Onsager [19], a "violet catastrophe." It is a consequence of these divergences that the fluid equations (1)-(3) can no longer remain meaningful in the naive sense because they involve the above diverging gradients. To make sense of the dynamics in the ideal limit, Onsager used a point-splitting regularization partially smoothed by a filtering kernel [31]. We employ an alternative approach $[40,41]$ more closely related to renormalization-group (RG) methods, with fields $f(\mathbf{x}, t)$ spatially coarse grained as

$$
\bar{f}_{\ell}(\mathbf{x}, t)=\int d^{d} r G_{\ell}(\mathbf{r}) f(\mathbf{x}+\mathbf{r}, t),
$$

where $G_{\ell}(\mathbf{r})=\ell^{-d} G(\mathbf{r} / \ell)$ and the filter kernel $G$ is nonnegative, smooth, rapidly decreasing in space, and normalized so that $\int d^{d} r G(\mathbf{r})=1$. This coarse-graining operation is a natural regularization that removes short-distance divergences. This can be seen from Cauchy-Schwartz bounds on the coarse-grained gradients:

$$
\left|\boldsymbol{\nabla} \bar{f}_{\ell}\right| \leq(1 / \ell) \sqrt{\int d^{d} r\left|(\boldsymbol{\nabla} G)_{\ell}(\mathbf{r})\right|^{2} \cdot \int_{\operatorname{supp}\left(G_{\ell}\right)} d^{d} r f^{2}(\mathbf{x}+\mathbf{r})},
$$

which are finite as long as the function $f$ is locally square integrable. Such estimates are intuitively obvious because high wave numbers $k \gtrsim 1 / \ell$ have been removed. As we shall see, the coarse-graining regularization in Eq. (23) is more powerful and more general than the point splitting originally used by Kolmogorov and Onsager [85]. The coarse-grained field in Eq. (23) is analogous to a "block spin" in a Wilson-Kadanoff RG scheme [87,88]. A crucial point is that the coarse graining is a purely passive operation, which corresponds simply to "taking off one's spectacles." Although it smooths out divergences, no objective physical phenomenon can depend on the arbitrary scale $\ell$ of coarse graining. In this work, we shall draw important conclusions from this independence, which is a type of nonperturbative RG invariance [49]. To keep our notations simple, we denote $\bar{f}_{\ell}$ as $\bar{f}$, unless it is essential that the length scale $\ell$ be made explicit.

Not only do the equations of motion of coarse-grained fluid variables remain well defined in the ideal limit, but another crucial fact is that dissipative transport in those equations due to the molecular coefficients becomes negligible at fixed length scale $\ell$ in the limit $\eta, \zeta$, $\kappa \rightarrow 0$. Because this is an essential point, we give a simple proof of this fact here. Starting with momentum transport, a coarse graining of the momentum balance equation yields 


$$
\partial_{t}(\overline{\rho \mathbf{v}})+\boldsymbol{\nabla} \cdot(\overline{\rho \mathbf{v v}+p \mathbf{I}-2 \eta \mathbf{S}-\zeta \Theta \mathbf{I}})=\mathbf{0}
$$

because coarse graining commutes with space and time derivatives. The contribution of the shear viscosity can be bounded pointwise using the Cauchy-Schwartz inequality

$$
\begin{aligned}
|\boldsymbol{\nabla} \cdot \overline{2 \eta \mathbf{S}}(\mathbf{x}, t)| \\
\quad=\frac{2}{\ell}\left|\int d^{3} r(\boldsymbol{\nabla} G)_{\ell}(\mathbf{r}) \cdot \eta(\mathbf{x}+\mathbf{r}, t) \mathbf{S}(\mathbf{x}+\mathbf{r}, t)\right| \\
\quad \leq \frac{2}{\ell} \sqrt{\langle\eta(\mathbf{x})\rangle_{\ell} \times \int d^{3} r\left|(\boldsymbol{\nabla} G)_{\ell}(\mathbf{r})\right|^{2} \eta(\mathbf{x}+\mathbf{r}, t)|\mathbf{S}(\mathbf{x}+\mathbf{r}, t)|^{2}}
\end{aligned}
$$

with $\langle\eta(\mathbf{x}, t)\rangle_{\ell}=\int_{\operatorname{supp} G_{\ell}} d^{3} r \eta(\mathbf{x}+\mathbf{r}, t)$ and with $\operatorname{supp}\left(G_{\ell}\right)$ the compact support set of the function $G_{\ell}$. The inverse power $1 / \ell$ arose after using integration by parts to move the gradient to the filter function $G$. It shows that this term is essentially "irrelevant" in the RG sense and is damped out for increasing $\ell$. When $G$ is a test function in the Schwartz distribution theory $\left(G \in C^{\infty}\right.$ and compactly supported), then so is $|\boldsymbol{\nabla} G(\cdot-\mathbf{x})|^{2}$; thus,

$$
\begin{aligned}
& \lim _{\eta, \zeta, K \rightarrow 0} \int d^{3} r\left|(\boldsymbol{\nabla} G)_{\ell}(\mathbf{r})\right|^{2} \eta(\mathbf{x}+\mathbf{r}, t)|\mathbf{S}(\mathbf{x}+\mathbf{r}, t)|^{2} \\
& \quad=\int\left|(\nabla G)_{\ell}(\mathbf{r}-\mathbf{x})\right|^{2} Q_{\eta}(d \mathbf{r})
\end{aligned}
$$

by our fundamental hypothesis. On the other hand, $\langle\eta(\mathbf{x})\rangle_{\ell} \rightarrow 0$ whenever $\eta$ tends to zero locally in $L^{1}$. An identical argument also shows that $|\nabla \overline{\zeta \Theta}(\mathbf{x}, t)| \rightarrow 0$ pointwise for fixed length scale $\ell$ when $\zeta$ tends to zero locally in $L^{1}$. It follows that all of the molecular transport terms in the coarse-grained momentum balance become negligible in the limit of high Reynolds numbers.

This leads to the concept of the "inertial range," or the length scales $\ell$ sufficiently large that the molecular transport can be ignored relative to the large-scale momentum transport of about $\rho_{0} v_{0}^{2} / L_{0}$. The previous upper bound shows that this range extends down to at least $\ell \sim L_{0} / \sqrt{\operatorname{Re}_{s}}$ with a Reynolds number defined by $1 / \operatorname{Re}_{s}=\eta_{0} Q_{\eta} / \rho_{0}^{2} v_{0}^{4}$, which is analogous to the "Taylor microscale" of incompressible fluid turbulence. Here, we have assumed that $\zeta \sim \eta$; otherwise, one must also consider the limit set by $\ell \gtrsim L_{0} / \sqrt{\operatorname{Re}_{b}}$, with another "bulk-viscosity Reynolds number" defined by $1 / \operatorname{Re}_{b}=\zeta_{0} Q_{\eta} / \rho_{0}^{2} v_{0}^{4}$. It should be emphasized that the above estimates of length scales where viscosity effects become significant are expected to be overestimates because they are deduced from mathematical upper bounds on the molecular transport. The range of scales $\ell$ where viscosity is significant is usually termed the "dissipation range" and extends down to scales of order the mean-free-path length $\lambda_{\text {mfp }}$ of the fluid, where the hydrodynamic description breaks down.

The same arguments apply also to the energy balance, where the shear-viscosity contribution is bounded by

$$
\begin{aligned}
\mid \boldsymbol{\nabla} \cdot & \overline{2 \eta \mathbf{S} \cdot \mathbf{v}}(\mathbf{x}, t) \mid \\
\leq & \frac{2}{\ell} \sqrt{\int_{\text {supp } G_{\ell}} d^{3} r|\mathbf{v}(\mathbf{x}+\mathbf{r})|^{2} \eta(\mathbf{x}+\mathbf{r}, t)} \\
& \times \sqrt{\int d^{3} r\left|(\mathbf{\nabla} G)_{\ell}(\mathbf{r})\right|^{2} \eta(\mathbf{x}+\mathbf{r}, t)|\mathbf{S}(\mathbf{x}+\mathbf{r}, t)|^{2}},
\end{aligned}
$$

and this vanishes at fixed $\ell$, for example, if $\mathbf{v}$ is locally $L^{2}$ and if $\eta$ tends to zero locally in $L^{\infty}$. The energy transport by shear viscosity is negligible compared with large-scale advective transport of about $\rho_{0} v_{0}^{3} / L_{0}$, again down to length scale $\ell \sim L_{0} / \sqrt{\operatorname{Re}_{s}}$ (at least). Similar arguments apply to the other molecular contributions to energy transport. The contribution from bulk viscosity tends to zero if $\zeta \rightarrow 0$ locally in $L^{\infty}$ and is negligible down to at least the length scale $\ell \sim L_{0} / \sqrt{\operatorname{Re}_{b}}$. Finally, the contribution from thermal conductivity vanishes if temperature $T$ is locally $L^{2}$ and $\kappa \rightarrow 0$ locally in $L^{\infty}$, and it may be neglected down to at least length scale $\ell \sim L_{0} / \sqrt{\mathrm{Pe}_{c}}$ for the thermal Péclet number defined by $1 / \mathrm{Pe}_{c}=\kappa_{0} T_{0}^{2} \Sigma_{\text {therm }} / \rho_{0}^{2} v_{0}^{6}$.

The final conclusion of this argument is that, for sufficiently large length scales $\ell$ (or for all $\ell$ in the ideal limit $\eta, \zeta, \kappa \rightarrow 0$ ), the following set of coarse-grained balance equations holds:

$$
\begin{gathered}
\partial_{t} \bar{\rho}+\boldsymbol{\nabla} \cdot(\overline{\rho \mathbf{v}})=0, \\
\partial_{t}(\overline{\rho \mathbf{v}})+\boldsymbol{\nabla} \cdot(\overline{\rho \mathbf{v v}+p \mathbf{I}})=\mathbf{0}, \\
\partial_{t}\left(\overline{\frac{1}{2} \rho v^{2}+u}\right)+\boldsymbol{\nabla} \cdot\left[\overline{\left(u+p+\frac{1}{2} \rho v^{2}\right) \mathbf{v}}\right]=0,
\end{gathered}
$$

in which the molecular transport terms are absent. This set of equations for all $\mathbf{x}, t$, and $\ell$ is mathematically equivalent to the statement that any limiting fields $\rho, \mathbf{v}, u$ are distributional or "weak" solutions of the compressible Euler equations (see Ref. [89], Sec. 4; and Ref. [90]). It must be appreciated that this notion of "distributional or weak solution" is quite distinct from the statement that either the fields $\rho, \mathbf{v}, u$ or their coarse-grained versions satisfy compressible Euler equations in the usual naive sense [91]. This point can be made clearly by introducing the density-weighted Favre average $[93,95]$,

$$
\tilde{f}=\overline{\rho f} / \bar{\rho}
$$

and using the expansion of average products $\widetilde{f_{1} \ldots f_{n}}$ into a finite sum of $p$ th-order cumulants $\tilde{\tau}\left(f_{i_{1}}, \ldots, f_{i_{p}}\right)$ : 


$$
\widetilde{f_{1} \ldots f_{n}}=\sum_{I} \prod_{r=1}^{r_{I}} \tilde{\tau}\left(f_{i_{1}^{(r)}}, \ldots, f_{i_{p r}^{(r)}}\right)
$$

where the sum is over all distinct partitions $I$ of $\{1, \ldots, n\}$ into $r_{I}$ disjoint subsets $\left\{i_{1}^{(r)}, \ldots, i_{p_{r}}^{(r)}\right\}, r=1, \ldots, r_{I}$, so that $\sum_{r=1}^{r_{I}} p_{r}=n$ for each partition $I[96,97]$. One may likewise expand averaged products $\overline{f_{1} \ldots f_{n}}$ into cumulants $\bar{\tau}\left(f_{1}, \ldots, f_{n}\right)$ for the original (nondensity-weighted) spatial coarse graining. Exploiting these cumulant expansions in the ideal balance equations yields an equivalent set of equations,

$$
\begin{gathered}
\partial_{t} \bar{\rho}+\boldsymbol{\nabla} \cdot(\bar{\rho} \tilde{\mathbf{v}})=0, \\
\partial_{t}(\bar{\rho} \tilde{\mathbf{v}})+\boldsymbol{\nabla} \cdot(\bar{\rho} \tilde{\mathbf{v}} \tilde{\mathbf{v}}+\bar{\rho} \tilde{\tau}(\mathbf{v}, \mathbf{v})+\bar{p} \mathbf{I})=\mathbf{0}, \\
\partial_{t}\left(\frac{1}{2} \bar{\rho}|\tilde{\mathbf{v}}|^{2}+\frac{1}{2} \bar{\rho} \tilde{\tau}\left(v_{i}, v_{i}\right)+\bar{u}\right) \\
+\boldsymbol{\nabla} \cdot[(\bar{u}+\bar{p}) \overline{\mathbf{v}}+\bar{\tau}(u+p, \mathbf{v}) \\
\left.+\frac{1}{2} \bar{\rho}|\tilde{\mathbf{v}}|^{2} \tilde{\mathbf{v}}+\bar{\rho} \tilde{v}_{i} \tilde{\tau}\left(v_{i}, \mathbf{v}\right)+\frac{1}{2} \bar{\rho} \tilde{\tau}\left(v_{i}, v_{i}, \mathbf{v}\right)\right]=0 .
\end{gathered}
$$

It is immediately clear that the coarse-grained fields $\bar{\rho}$, $\tilde{\mathbf{v}}=\overline{\mathbf{j}} / \bar{\rho}$, and $\bar{u}$, although smooth and with all derivatives well defined, do not satisfy the compressible Euler equations in the standard sense and that there are new transport terms at length scale $\ell$ that were introduced by the coarse graining. Of course, it is not surprising that the effective equations for block-spin variables are renormalized and contain new, complex terms. Note, in particular, that the coarse-graining cumulants of second and higher orders are not simple closed functions of the "resolved" fields $\bar{\rho}, \tilde{\mathbf{v}}, \bar{u}$. The cumulants are instead very complex functions of the resolved fields, with nonpolynomial nonlinearity and nonMarkovian dependence on the past history. In fact, these cumulants cannot, in principle, be fixed, deterministic functions of the resolved fields, but they must be considered stochastic [98] variables because of their dependence on the unknown degrees of freedom below length scale $\ell$ [66]. In the "large-eddy simulation" (LES) methodology of turbulence modeling, one seeks computationally tractable closed models of these cumulant terms as functions of the resolved fields (see Refs. [94,101,102]). As we shall see, the cumulant terms that appear in these coarse-grained equations are the source of turbulent cascade and dissipative anomalies for weak Euler solutions [40].

The above description of weak solutions is somewhat novel and designed to make clear the close connection with renormalization-group methodology. A more traditional account follows by first taking the ideal limit $\eta, \zeta, \kappa \rightarrow 0$ of the coarse-grained conservation equations, just as above, and then followed by the limit $\ell \rightarrow 0$.
The coarse-grained balance equations in this order of limits converge in the sense of distributions to

$$
\begin{gathered}
\partial_{t} \rho+\boldsymbol{\nabla} \cdot(\rho \mathbf{v})=0, \\
\partial_{t}(\rho \mathbf{v})+\boldsymbol{\nabla} \cdot(\rho \mathbf{v} \mathbf{v}+p \mathbf{I})=\mathbf{0}, \\
\partial_{t}\left(\frac{1}{2} \rho v^{2}+u\right)+\boldsymbol{\nabla} \cdot\left[\left(u+p+\frac{1}{2} \rho v^{2}\right) \mathbf{v}\right]=0 .
\end{gathered}
$$

This system follows because all space and time derivatives can be transferred to the test functions and all coarsegrained fields inside the derivatives converge to their fine-grained values under modest assumptions on the fields (e.g., if they are bounded, measurable functions). Equivalently, all of the coarse-graining cumulants of the fields converge to zero. In contrast to the regularized systems of equations (29)-(31) or (34)-(36) where all derivatives are taken in the classical sense, in the above set of $\ell \rightarrow 0$ limit equations (37)-(39), the derivatives must be interpreted distributionally since the limit functions $\rho, \mathbf{v}, u$ are not generally even once differentiable. (See further discussion on fluid singularities below.) This more conventional notion of a weak solution is a suitable mathematical idealization of infinite Reynolds-number turbulence, where the inertial range extends to infinitesimally small scales. The concept goes back to Onsager [20], who termed it "ideal turbulence."

As we now discuss, standard consequences of the Euler equations for smooth "strong" solutions do not generally hold for weak solutions, which are instead afflicted with dissipative anomalies due to turbulent cascade. First, we make an important comment on notations. Whenever coarse-grained quantities marked with $\overline{(\cdots)}$ or $(\widetilde{\cdots)}$ appear hereafter, we assume that the ideal limit $\eta, \zeta, \kappa \rightarrow 0$ has been taken, unless indicated otherwise (e.g., by explicitly taking this limit, or by retaining terms with explicit dependence on $\eta, \zeta, \kappa)$. This convention for coarse-grained quantities simplifies the expressions involved by eliminating the terms that vanish in the ideal limit by the arguments given above.

\section{ENERGY CASCADE}

\section{A. Kinetic energy}

We begin with kinetic-energy cascade. Because $|\mathbf{v}|^{2}$ is a convex function of $\mathbf{v}$, one has

$$
\frac{1}{2} \bar{\rho}|\tilde{\mathbf{v}}|^{2} \leq \frac{1}{2} \bar{\rho}|\widetilde{\mathbf{v}}|^{2}=\frac{1}{2} \overline{\rho|\mathbf{v}|^{2}} .
$$

Thus, the integral over space of $\frac{1}{2} \bar{\rho}|\tilde{\mathbf{v}}|^{2}$ is less than the total kinetic energy and represents only the "resolved" kinetic energy, while the second-order Favre cumulant 


$$
\frac{1}{2} \bar{\rho} \tilde{\tau}\left(v_{i}, v_{i}\right)=\frac{1}{2} \bar{\rho}\left[\tilde{v_{i}^{2}}-\tilde{v}_{i}^{2}\right] \geq 0
$$

represents the "unresolved" or "subscale" kinetic energy. In a decaying turbulence without external forcing, the finegrained kinetic energy integrated over space decreases because of the effect of viscosity. Since

$$
\frac{1}{2} \int d^{d} x \bar{\rho}|\tilde{\mathbf{v}}|^{2} \leq \frac{1}{2} \int d^{d} x \rho|\mathbf{v}|^{2},
$$

this decrease must also occur with increasing time for the resolved kinetic energy, despite the negligible effect of viscosities for $\ell$ in the inertial range. Physically speaking, the kinetic energy will decay whether an observer is "wearing spectacles" or not. The following question thus arises: How can the resolved kinetic-energy decay, if not through the influence of viscosity? A similar question arises for forced steady states. If the fluid is stirred by a large-scale acceleration field, then it is not hard to show that the input of resolved kinetic energy is nearly independent of $\ell$ for $\ell \lesssim L$, the length scale of the acceleration field (Ref. [23], Appendix B). What mechanism is available at length scales $\ell$ in the inertial range in order to dispose of the fixed mechanical power input and to maintain a mean steady-state energy?

An obvious answer is that the cumulant term in Eq. (35), or the "subscale stress" $\tilde{\tau}(\mathbf{v}, \mathbf{v})$, provides an effective dissipation of kinetic energy for $\ell$ in the inertial range. This can be seen from the balance equation for the resolved kinetic energy, which may be easily calculated from Eq. $(35)$ to be $[23,38]$

$$
\begin{aligned}
\partial_{t}( & \left.\frac{1}{2} \bar{\rho}|\tilde{\mathbf{v}}|^{2}\right) \\
& +\boldsymbol{\nabla} \cdot\left[\left(\bar{p}+\frac{1}{2} \bar{\rho}|\tilde{\mathbf{v}}|^{2}\right) \tilde{\mathbf{v}}+\bar{\rho} \tilde{\tau}(\mathbf{v}, \mathbf{v}) \cdot \tilde{\mathbf{v}}-\frac{\bar{p}}{\bar{\rho}} \bar{\tau}(\rho, \mathbf{v})\right] \\
= & \bar{p}(\boldsymbol{\nabla} \cdot \overline{\mathbf{v}})-\frac{\boldsymbol{\nabla} \bar{p}}{\bar{\rho}} \cdot \bar{\tau}(\rho, \mathbf{v})+\rho \boldsymbol{\nabla} \tilde{\mathbf{v}}: \tilde{\tau}(\mathbf{v}, \mathbf{v}) .
\end{aligned}
$$

The final term on the right-hand side is the so-called "deformation work" or the work done by the large-scale velocity-gradient $\boldsymbol{\nabla} \tilde{\mathbf{v}}$ acting against the subscale stress $\tilde{\tau}(\mathbf{v}, \mathbf{v})$. It is thus a typical "fluxlike term" describing an interaction between resolved and unresolved degrees of freedom and, on average, transferring kinetic energy from resolved to unresolved modes. This term is one of the main contributors to kinetic-energy cascade. It is more traditional to combine the first two terms on the right-hand side of Eq. (43) into a single term $\bar{p}(\boldsymbol{\nabla} \cdot \tilde{\mathbf{v}})$ [94] while also dropping the last term in the square brackets (representing space transport of kinetic energy) on the left-hand side. Above, we have followed Aluie [23,38] in separating the contributions of resolved pressure work $\bar{p}(\boldsymbol{\nabla} \cdot \overline{\mathbf{v}})$ and "baropycnal work" $-\boldsymbol{\nabla} \bar{p} \cdot \bar{\tau}(\rho, \mathbf{v}) / \bar{\rho}$, using the simple relation

$$
\tilde{\mathbf{v}}=\overline{\mathbf{v}}+\frac{1}{\bar{\rho}} \bar{\tau}(\rho, \mathbf{v}) .
$$

This division was motivated physically in Refs. [23,38], which pointed out that the resolved pressure work is a purely large-scale quantity, whereas the baropycnal work is "fluxlike" and describes an interaction between the resolved pressure gradient and subscale mass transport. For more discussion of the physics of this term, see Refs. [23,38]. The baropycnal work is thus an additional contributor to kinetic-energy cascade, with total inertial-range energy flux represented by the combination

$$
Q_{\ell}^{\mathrm{flux}}=\frac{\boldsymbol{\nabla} \bar{p}}{\bar{\rho}} \cdot \bar{\tau}(\rho, \mathbf{v})-\bar{\rho} \boldsymbol{\nabla} \tilde{\mathbf{v}}: \tilde{\tau}(\mathbf{v}, \mathbf{v}) .
$$

As we see presently, there are also compelling mathematical reasons to make the above separation of the pressure work.

The cascade terms in Eq. (43) are a possible source of the dissipative anomaly of kinetic energy for the weak solutions of Euler obtained in the limit first $\eta, \zeta, \kappa \rightarrow 0$ and then $\ell \rightarrow 0$. Taking the limit $\ell \rightarrow 0$ of the balance equation (43), one obtains

$\partial_{t}\left(\frac{1}{2} \rho v^{2}\right)+\boldsymbol{\nabla} \cdot\left[\left(p+\frac{1}{2} \rho v^{2}\right) \mathbf{v}\right]=p \circ \Theta-Q_{\text {flux }}$,

which is the kinetic-energy balance for the limiting weak Euler solution. Here, we defined

$$
p \circ \Theta=\mathcal{D}-\lim _{\ell \rightarrow 0} \bar{p} \cdot \bar{\Theta}
$$

with $\Theta=\boldsymbol{\nabla} \cdot \mathbf{v}$ and

$$
Q_{\text {flux }}=\mathcal{D} \text { - } \lim _{\ell \rightarrow 0} Q_{\ell}^{\text {flux }},
$$

where $\mathcal{D}$ - lim denotes the limit in the sense of distributions. We now discuss the physical meaning of these two terms.

Because $p$ and $\mathbf{v}$ are not generally smooth functions, the divergence $\Theta$ exists only as a distribution, and its product with the nonsmooth function $p$ is thus ill defined and ambiguous. The limit $p \circ \Theta$ in Eq. (47) is a standard approach to define a generalized product of distributions [103], and our circle notation "o" is meant to emphasize that this product must be carefully defined. Despite this subtlety, however, such a term is exactly the same as that which appears for a smooth Euler solution. It clearly represents pressure work in the large scales, which converts energy from mechanical to internal and vice versa. The tendency to equipartition of total energy suggests that, when the turbulence is mechanically forced, the transfer will be, on average, from mechanical to internal. It was already argued in Refs. [23,38] that the mean transfer $\left\langle\bar{p}_{\ell} \bar{\Theta}_{\ell}\right\rangle$ at length scales greater than $\ell$ will saturate to a constant negative value as $\ell$ decreases through the inertial 
range, and this saturation has been verified in numerical simulations of subsonic and transonic compressible turbulence $[104,105]$. Our mathematical analysis implies that $\langle p \circ \Theta\rangle<0$ will give the saturated level.

The additional term $Q_{\text {flux }}$ that appears in Eq. (46) is, on the other hand, entirely missing for smooth Euler solutions and represents a kinetic-energy anomaly. It is due to the loss of kinetic energy by turbulent cascade to infinitesimally small scales. As shown by Onsager [20,31] for the case of incompressible fluid turbulence, the nonvanishing of such a cascade term requires singularities of the fluid fields $\rho, \mathbf{v}$, and $p$ [106]. For a complete proof of the analogous result for compressible fluids, see the works of Aluie $[23,38,108]$ and the companion paper [90]. Briefly, the result follows by expanding Favre averages into cumulants $\bar{\tau}\left(f_{1}, \ldots, f_{n}\right)$ of the original (nondensity-weighted) coarse graining. A fundamental fact is that such cumulants and their spatial gradients can be written entirely in terms of space increments $\delta f_{i}(\mathbf{r} ; \mathbf{x})=f_{i}(\mathbf{x}+\mathbf{r})-f_{i}(\mathbf{x})$ of the fields $f_{i}$ (for a proof, see Ref. [89], Appendix B, or Ref. [109]). From these basic identities, one can derive estimates of the form

$$
\begin{array}{r}
\boldsymbol{\nabla} \tilde{\mathbf{v}}=\frac{O(\delta v)}{\ell}\left[1+O\left(\frac{\delta \rho}{\rho}\right)+O\left(\left(\frac{\delta \rho}{\rho}\right)^{2}\right)\right], \\
\tilde{\tau}(\mathbf{v}, \mathbf{v})=O(\delta v)^{2}\left[1+O\left(\frac{\delta \rho}{\rho}\right)+O\left(\left(\frac{\delta \rho}{\rho}\right)^{2}\right)\right],
\end{array}
$$

where $\delta v, \delta \rho$ denote increments over the length scale $\ell$. For example, see Eqs. (1) and (2) and intervening relations in the paper of Aluie [38]. Substituting such expressions into the formula for the deformation work yields an analogue for compressible turbulence of the 4/5th law of Kolmogorov [17], which expresses the energy flux in terms of increments of the basic fields [110]. One can easily see that when the fields $\mathbf{v}$ and $\rho$ are space differentiable, then $\delta v, \delta \rho=O(\ell)$, and thus energy flux due to deformation work vanishes at least as $O\left(\ell^{2}\right)$ for $\ell \rightarrow 0$. In order to sustain a nonvanishing energy flux, the fluid variables must appear "rough" for $\ell$ in the inertial range. A more precise statement is that the scaling exponents $\zeta_{p}^{v}$ of the $q$ th-order (absolute) velocity structure functions [37] must be sufficiently small. For example, when density $\rho$ is bounded away from zero and infinity, then nonvanishing of $\lim _{\ell \rightarrow 0} \bar{\rho} \boldsymbol{\nabla} \tilde{\mathbf{v}}: \tilde{\tau}(\mathbf{v}, \mathbf{v})$ requires

$$
\zeta_{q}^{v} \leq q / 3, \quad \forall q \geq 3,
$$

where $q / 3$ is the dimensional Kolmogorov value. See Refs. [90,108]. This is an exact singularity statement for the velocity field in compressible turbulence, consistent with possible spatial intermittency. It is interesting to note that for structure-function exponents in the range $0<\zeta_{q}^{v}$, $\zeta_{q}^{\rho}<p$, the deformation work can be shown to be scale local [38,111], and thus "cascade" is an apt description of the transfer process [112].

Similar results also hold for the baropycnal work, where identical arguments yield a 4/5th-law type result of the form $[23,38,90]$

$$
-\nabla \bar{p} \cdot \bar{\tau}(\rho, \mathbf{v}) / \bar{\rho}=O\left(\frac{(\delta p)(\delta \rho)(\delta v)}{\ell \rho}\right) .
$$

An Onsager singularity theorem for this quantity states that it can be nonvanishing as $\ell \rightarrow 0$ only if a condition is satisfied of the form

$$
\zeta_{q}^{p}+\zeta_{q}^{\rho}+\zeta_{q}^{v} \leq q, \quad \forall q \geq 3
$$

for scaling exponents of all three fields $p, \rho, \mathbf{v}[90,108]$. Thus, the baropycnal work contributes to energy cascade only if the pressure and density, in addition to the velocity, are sufficiently rough. When the $q$ th-order scaling exponents of all these fields lie between 0 and $q$, then the baropycnal work is also a scale-local quantity by the same arguments as for deformation work [38,111]. These inequalities have been stated in terms of singularities for Euler solutions obtained in the limit of infinite Reynolds numbers, but it is important to emphasize that Eqs. (51) and (53) are necessary for sustaining an energy cascade rate nondecreasing with $\ell$ at large but finite Reynolds numbers.

The above conclusions on kinetic-energy cascade in compressible turbulence are almost entirely based upon earlier works of Aluie $[23,38,108]$ and seem to closely parallel the theory of Onsager for incompressible fluids. In fact, we exactly recover his results [20,31], by setting $\rho=$ (const) and $\Theta=0$. However, we now show that compressibility leads to a novel mechanism for anomalous dissipation of kinetic energy. By our fundamental hypothesis (18), the viscous heating does not vanish at high Reynolds numbers. It follows by taking the ideal limit $\eta, \zeta$, $\kappa \rightarrow 0$ of the fine-grained kinetic-energy balance (14) that

$\partial_{t}\left(\frac{1}{2} \rho v^{2}\right)+\boldsymbol{\nabla} \cdot\left[\left(p+\frac{1}{2} \rho v^{2}\right) \mathbf{v}\right]=p * \Theta-Q_{\mathrm{visc}}$,

where $Q_{\text {visc }}=Q_{\eta}+Q_{\zeta}$ as in Eq. (18) and we have defined

$$
p * \Theta=\mathcal{D}-\lim _{\eta, \zeta, k \rightarrow 0} p \Theta .
$$

One might naively conjecture that the latter quantity is the same as $p \circ \Theta$ given by Eq. (47). However, the general theory of distributional products makes this a priori highly unlikely. It is part of the definition of the product $f \circ g=$ $\mathcal{D}-\lim _{\ell \rightarrow 0} \bar{f}_{\ell} \cdot \bar{g}_{\ell}$ that the limiting distribution must be independent of precisely which filter kernel $G$ is employed, but it is generally not true that other regularizations $f_{\epsilon}, g_{\epsilon}$ for which $f_{\epsilon} \stackrel{\mathcal{D}}{\rightarrow} f, g_{\epsilon} \stackrel{\mathcal{D}}{\rightarrow} g$ will have the same limiting product 
$f_{\epsilon} \cdot g_{\epsilon} \stackrel{\mathcal{D}}{\rightarrow} f \circ g$ [103]. Since viscosities and thermal conductivities are a different "regularization" of the Euler system than mere coarse graining, one should expect that $p * \Theta \neq p \circ \Theta$.

Nevertheless, the fine-grained or dissipation-range energy balance (54) must agree with the coarse-grained inertial-range balance (46) in the limit as $\ell \rightarrow 0$. Objective physical facts such as the rate of decay of energy or the rate of absorption of power input cannot depend upon an arbitrary scale $\ell$ of spatial resolution of observations (cf. Ref. [49]). After first taking the limit $\eta, \zeta, \kappa \rightarrow 0$, we must then be able to take $\ell \rightarrow 0$ and reproduce the same result. Comparing Eqs. (54) and (46), it follows that

$$
Q_{\text {visc }}=\tau(p, \Theta)+Q_{\text {flux }},
$$

where we have defined the quantity

$$
\begin{aligned}
\tau(p, \Theta) & =\mathcal{D}-\lim _{\ell \rightarrow 0} \lim _{\eta, \zeta, \kappa \rightarrow 0} \bar{\tau}(p, \Theta) \\
& =p * \Theta-p \circ \Theta
\end{aligned}
$$

which we call the "pressure-dilatation defect." It is nonvanishing when the joint $\operatorname{limits} \lim _{\ell \rightarrow 0}$ and $\lim _{\eta, \zeta, \kappa \rightarrow 0}$ of the product $\bar{p} \cdot \bar{\Theta}$ do not commute but instead yield either $p * \Theta$ or $p \circ \Theta$ depending upon the order of the two limits. Unlike incompressible fluid turbulence where $Q_{\text {visc }}=Q_{\text {flux }}$ [42], we see that, for compressible fluids, the pressure-dilatation defect $\tau(p, \Theta)$ can be another source of anomalous dissipation distinct from energy cascade.

In fact, all stationary, planar shocks in fluids with an ideal-gas equation of state exhibit this mechanism in a pure form because there $Q_{\text {flux }}=0$ and $Q_{\text {visc }}=\tau(p, \Theta) \geq 0$. For a proof of this result, see Appendix A. All of the anomalous dissipation in such shocks, or so-called "shock heating," is due to the pressure-dilatation defect. In addition to the general inertial-range result that $Q_{\text {flux }}=0$ for such shocks, we can also obtain exact dissipation-range limits at special values of the Prandtl number where analytical results are available: $\operatorname{Pr}=3 / 4$ [114] and $\operatorname{Pr}=0, \infty$ [115]. For cases $\operatorname{Pr}=3 / 4, \infty$, in particular, we show in Appendix A that $\tau(p, \Theta)>0$.

Our arguments show generally that $Q_{\text {visc }}>0$ only if at least one of $\tau(p, \Theta)$ or $Q_{\text {flux }}$ is positive. For developed compressible turbulence, one should expect that both of these mechanisms will contribute. At finite $\ell$, we may rewrite the inertial-range kinetic-energy balance (43) as

$$
\begin{aligned}
\partial_{t}\left(\frac{1}{2} \bar{\rho}|\tilde{\mathbf{v}}|^{2}\right)+\boldsymbol{\nabla} \cdot\left[\left(\bar{p}+\frac{1}{2} \bar{\rho}|\tilde{\mathbf{v}}|^{2}\right) \tilde{\mathbf{v}}\right. \\
\left.\quad+\bar{\rho} \tilde{\tau}(\mathbf{v}, \mathbf{v}) \cdot \tilde{\mathbf{v}}-\frac{\bar{p}}{\bar{\rho}} \bar{\tau}(\rho, \mathbf{v})\right]=\overline{p * \Theta}-Q_{\ell}^{\text {inert }}
\end{aligned}
$$

where

$$
Q_{\ell}^{\text {inert }}=\bar{\tau}_{\ell}(p, \Theta)+Q_{\ell}^{\text {flux }}
$$

is an effective "inertial dissipation" at scale $\ell$, such that $Q^{\text {inert }}:=\mathcal{D}$ - $\lim _{\ell \rightarrow 0} Q_{\ell}^{\text {inert }}=Q_{\text {visc }}$. The effective dissipation at each arbitrary scale $\ell$ can agree with the fine-grained or viscous dissipation rate only if there is either nonlinear energy cascade [116] with $Q_{\ell}^{\text {flux }}>0$ or a positive defect $\bar{\tau}_{\ell}(p, \Theta)>0$ as $\ell \rightarrow 0$. The estimates of Aluie [23,38,108] show that the fluid variables must be sufficiently rough in order to sustain energy cascade. The consequences (51) and (53) for scaling exponents are directly testable predictions of the argument, which is an exact, nonperturbative application of the principle of renormalization-group invariance [46-48]. Although it is not yet obvious, the condition that $\bar{\tau}_{\ell}(p, \Theta)>0$ as $\ell \rightarrow 0$ also requires these same exponent relations to hold. In order to show this, we must develop a deeper understanding of the thermodynamics of compressible turbulence.

\section{B. Internal energy}

The other half of the energy budget is internal energy. Numerical results $[104,105]$ show that up to $50 \%$ of the energy injected at large scales can be channeled into internal energy by the large-scale pressure work. We must therefore consider the inertial-range dynamics of internal energy. The simplest way to obtain an equation for the coarse-grained or resolved internal energy $\bar{u}$ is to apply the coarse-graining operation to Eq. (15) for fine-grained internal energy and to then consider the ideal (infinite Reynolds and Péclet number) limit. The first step yields

$$
\partial_{t} \bar{u}+\boldsymbol{\nabla} \cdot[\overline{u \mathbf{v}-\kappa \boldsymbol{\nabla} T}]=-\overline{p \Theta}+\overline{2 \eta|\mathbf{S}|^{2}+\zeta \Theta^{2}} .
$$

Invoking the fundamental hypothesis (18) and taking the ideal limit $\eta, \zeta, \kappa \rightarrow 0$ yields the following equation for the inertial-range dynamics of the internal energy:

$$
\partial_{t} \bar{u}+\boldsymbol{\nabla} \cdot(\bar{u} \overline{\mathbf{v}}+\bar{\tau}(u, \mathbf{v}))=-\overline{p * \Theta}+\bar{Q}_{\mathrm{visc}} .
$$

In the subsequent limit $\ell \rightarrow 0$, we get

$$
\partial_{t} u+\nabla \cdot(u \mathbf{v})=-p * \Theta+Q_{\text {visc }}
$$

as the distributional balance of internal energy for the limiting weak Euler solution.

On the other hand, we can obtain another form of this equation by subtracting the balance equation (43) for resolved kinetic energy from the coarse graining of Eq. (39) for conservation of total energy. After some straightforward calculations, this yields the equation 


$$
\begin{aligned}
\partial_{t}\left(\bar{u}+\frac{1}{2} \bar{\rho} \tilde{\tau}\left(v_{i}, v_{i}\right)\right) & +\boldsymbol{\nabla} \cdot\left(\bar{u} \overline{\mathbf{v}}+\bar{\tau}(h, \mathbf{v})+\frac{1}{2} \bar{\rho} \tilde{\tau}\left(v_{i}, v_{i}\right) \tilde{\mathbf{v}}\right. \\
\left.+\frac{1}{2} \bar{\rho} \tilde{\tau}\left(v_{i}, v_{i}, \mathbf{v}\right)\right) & =-\bar{p} \bar{\Theta}+Q_{\ell}^{\text {flux }},
\end{aligned}
$$

with $h=u+p$ the enthalpy. In this balance, the following quantity appears:

$$
\bar{u}^{*}:=\bar{u}+\frac{1}{2} \bar{\rho} \tilde{\tau}\left(v_{i}, v_{i}\right),
$$

which we call the intrinsic large-scale or resolved internal energy. It is a natural object because, based on coarsegrained observations alone, it is impossible to distinguish between energy in thermal fluctuations and that in unresolved turbulent fluctuations. In contrast to the balance (61) for resolved internal energy, the balance (63) for intrinsic large-scale internal energy contains no direct contributions from microscopic dissipation and is a consequence solely of the limiting distributional Euler solution. In the limit $\ell \rightarrow 0$, all of the cumulant terms in Eq. (63) vanish distributionally, and one obtains a second form of the internal-energy balance:

$$
\partial_{t} u+\nabla \cdot(u \mathbf{v})=-p \circ \Theta+Q_{\text {flux }} .
$$

Using Eq. (59), we may rewrite the right-hand side of

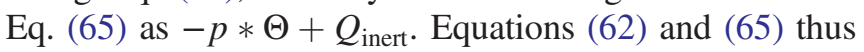
agree since $Q_{\text {visc }}=Q_{\text {inert }}=Q$. We see that the same $Q$ that appears as a sink in the kinetic-energy balance, Eq. (54) or (58), appears as a source in the balance of internal energy, Eq. (62) or (65).

One concern at this point is whether Eq. (61) for $\bar{u}$ truly represents "inertial-range dynamics," in contrast to Eq. (63) for $\bar{u}^{*}$, which is clearly an inertial-range balance. Quantities of the sort $\bar{Q}_{\text {visc }}$ have been much discussed for incompressible fluid turbulence in the context of the "Kolmogorov refined similarity hypothesis" [118], and it has been a debated issue whether such coarse-grained dissipation fields for inertial-range lengths $\ell$ should be considered inertial range or dissipation range. For example, Kraichnan [119] concluded that $\bar{Q}_{\text {visc }}$ is not inertial range: "Instead it is the integral of a dissipation-range quantity." The question is hard to argue substantively because there is no clear, accepted definition in the literature of what it means to be "inertial range" or "dissipation range." We would like to offer a simple, precise definition of an "inertial-range quantity" as any field that exists as an ordinary function (as opposed to a distribution only) in the ideal limit $\eta, \zeta, \kappa \rightarrow 0$. By this definition, $\bar{Q}_{\text {visc }}$ is clearly an inertial-range quantity and so is the pressure work $\overline{p * \Theta}$, although both involve effects of molecular dissipation that survive in the ideal limit $\eta, \zeta, \kappa \rightarrow 0$.

The questions about inertial-range status of $\bar{Q}_{\text {visc }}$ and $\overline{p * \Theta}$ cannot, however, be legitimately answered by merely offering a definition. The more serious worry that underlies this question is whether these can be universal quantities independent of the particular microscale dissipation mechanism, or whether they are distinct for every particular fine-grained dissipation (e.g., ordinary viscosity vs hyperviscosity). As a matter of fact, the quantities $\bar{Q}_{\text {visc }}$ and $\overline{p * \Theta}$ probably cannot be completely universal in compressible fluid turbulence, as they can be shown to be Prandtlnumber dependent [120] even for planar, ideal-gas shocks (see Appendix A). On the other hand, it is a direct consequence of Eq. (62) for the internal energy $u$ that the combination $-p * \Theta+Q_{\text {visc }}$ depends only upon the limiting Euler solution fields $\rho, \mathbf{v}, u$ and not upon the particular sequence $\eta, \zeta, \kappa \rightarrow 0$ used to obtain that solution. It is explicitly verified for planar, ideal-gas shocks in Appendix A that the combined quantity is independent of Prandtl number even though the quantities $\bar{Q}_{\text {visc }}$ and $\overline{p * \Theta}$ separately are $\operatorname{Pr}$ dependent. This suggests that the combination $\bar{Q}_{\text {visc }}-\overline{p * \Theta}$ for inertial-range length scales $\ell$ should be universal for a wide class of fine-grained dissipation mechanisms and determined only by fluid modes at scales comparable to $\ell$.

We can make a substantive argument for this assertion based upon the following equation for subscale or unresolved kinetic energy in the ideal limit:

$$
\begin{array}{r}
\partial_{t}\left(\frac{1}{2} \bar{\rho} \tilde{\tau}\left(v_{i}, v_{i}\right)\right)+\boldsymbol{\nabla} \cdot\left[\frac{1}{2} \bar{\rho} \tilde{\tau}\left(v_{i}, v_{i}\right) \tilde{\mathbf{v}}+\bar{\tau}(p, \mathbf{v})\right. \\
\left.+\frac{1}{2} \bar{\rho} \tilde{\tau}\left(v_{i}, v_{i}, \mathbf{v}\right)\right]=\bar{\tau}(p, \Theta)-\bar{Q}_{\text {visc }}+Q_{\ell}^{\text {flux }}
\end{array}
$$

This equation is straightforward to derive by considering the equations for $(1 / 2) \overline{\rho|\mathbf{v}|^{2}}$ and $(1 / 2) \bar{\rho}|\tilde{\mathbf{v}}|^{2}$, subtracting them, and taking the limit $\eta, \zeta, \kappa \rightarrow 0$. A simple reorganization of this equation gives

$$
\begin{aligned}
\overline{p * \Theta} & -\bar{Q}_{\text {visc }} \\
= & \bar{p} \bar{\Theta}-\bar{Q}_{\text {flux }}+\frac{1}{2} \bar{\rho}_{\tilde{D}} \tilde{\tau}\left(v_{i}, v_{i}\right) \\
& +\boldsymbol{\nabla} \cdot\left[\bar{\tau}(p, \mathbf{v})+\frac{1}{2} \bar{\rho} \tilde{\tau}\left(v_{i}, v_{i}, \mathbf{v}\right)\right],
\end{aligned}
$$

where $\tilde{D}_{t}=\partial_{t}+\tilde{\mathbf{v}} \cdot \boldsymbol{\nabla}$ is the large-scale Lagrangian time derivative. The important point is that all of the terms on the right-hand side of this equation are pure inertial-range quantities that are local in scale and thus determined only by fluid modes near the considered scale $\ell$. The standard arguments for universality thus apply to the right-hand side, so we may argue that, also on the left-hand side, the combination $\overline{p * \Theta}-\bar{Q}_{\text {visc }}$ will be a universal, inertial-range quantity, independent of the particular microscale mechanism of dissipation. As we discuss further in the following section, the above considerations play an essential role in our 
proof of a complete Onsager theorem for compressible turbulence [90]. By this, we mean the proof that a kineticenergy dissipation anomaly $Q \neq 0$ requires singularities in the fluid fields. As emphasized earlier, the arguments of Aluie $[23,38,108]$ imply that $Q_{\text {flux }} \neq 0$ requires the inequalities (51) and (53) to hold, but it is still possible, in principle, that $Q_{\text {visc }}=\tau(p, \Theta)>0$ with milder singularities.

Let us close this section by briefly considering the energy balances that must exist in a long-time steady state of mechanically forced compressible turbulence. In order for a steady state to exist, one must take into account cooling mechanisms, such as electromagnetic radiation; otherwise, the internal energy will continue to grow because of the input from viscous dissipation and mechanical work. The situation may be modeled by the compressible Navier-Stokes equations modified to include an external acceleration field $\mathbf{a}_{\mathrm{ext}}$ and a cooling function $Q_{\text {cool }}$ :

$$
\begin{gathered}
\partial_{t} \rho+\boldsymbol{\nabla} \cdot(\rho \mathbf{v})=0, \\
\partial_{t}(\rho \mathbf{v})+\boldsymbol{\nabla} \cdot(\rho \mathbf{v} \mathbf{v}+p \mathbf{I}-2 \eta \mathbf{S}-\zeta \Theta \mathbf{I})=\rho \mathbf{a}_{\mathrm{ext}}, \\
\partial_{t}\left(\frac{1}{2} \rho v^{2}+u\right)+\boldsymbol{\nabla} \cdot\left[\left(u+p+\frac{1}{2} \rho v^{2}\right) \mathbf{v}\right. \\
-\kappa \boldsymbol{\nabla} T-2 \eta \mathbf{S} \cdot \mathbf{v}-\zeta \Theta \mathbf{v}]=\rho \mathbf{v} \cdot \mathbf{a}_{\mathrm{ext}}-Q_{\mathrm{cool}} .
\end{gathered}
$$

The acceleration field is a source of mechanical input of kinetic energy $Q_{\text {in }}=\rho \mathbf{v} \cdot \mathbf{a}_{\text {ext }}$, whereas Eq. (15) for internal energy now includes the cooling term $-Q_{\text {cool }}$ on the right. When the forcing $\mathbf{a}_{\text {ext }}$ and cooling function $Q_{\text {cool }}$ are largescale (smooth) fields, then all of our previous considerations on the ideal limit apply. Steady-state kinetic-energy balance gives $\left\langle Q_{\text {in }}\right\rangle=\left\langle Q_{\text {trans }}\right\rangle+\langle Q\rangle$, where $Q_{\text {trans }}=$ $-p * \Theta$. From the fine-grained point of view, $Q=Q_{\text {visc }}$, whereas, in the inertial range, $Q=Q_{\text {inert }}$ and $\left\langle Q_{\text {inert }}\right\rangle=$ $\left\langle Q_{\text {in }}\right\rangle-\left\langle Q_{\text {trans }}\right\rangle$, exactly as argued earlier by Aluie [23]. The steady-state internal-energy balance likewise gives $\left\langle Q_{\text {trans }}\right\rangle+\langle Q\rangle=\left\langle Q_{\text {cool }}\right\rangle$, so $\left\langle Q_{\text {in }}\right\rangle=\left\langle Q_{\text {cool }}\right.$ in the steady state. In decaying turbulence without external forcing such as we considered throughout most of the paper, one expects a quasisteady state with initial conditions supplying the reservoir of energy to drive the cascade and $-\left\langle\partial_{t}\left(\rho v^{2} / 2\right)\right\rangle$ playing the role of $\left\langle Q_{\text {in }}\right\rangle$. Likewise, if cooling mechanisms are inefficient, then $\left\langle\partial_{t} u\right\rangle$ plays the role of $\left\langle Q_{\text {cool }}\right\rangle$.

\section{ENTROPY CASCADE}

The energy transfer $\bar{p} \bar{\Theta}$ from large-scale kinetic energy to large-scale internal energy $\bar{u}$, which was discussed in the previous section, does not represent a global heating of the fluid resulting merely in a uniform increase in the internal energy. It is instead an "ordered" or "coherent" input of energy, which leads to large-scale structure of the internal-energy field $\bar{u}$. One should thus expect this input to decrease the large-scale entropy of the system, which is maximum for a spatially homogeneous state. These considerations are one motivation to consider the entropy balance of the turbulent flow in detail, which allows us to verify the above expectations. In addition, entropy is an invariant of smooth Euler solutions but increases for viscous, heat-conducting fluids. Whereas kinetic energy need not evolve monotonically in time for a compressible fluid, the entropy is a quantity suitable to express the irreversibility in turbulent evolution of general fluids, both compressible and incompressible. The molecular entropy is also a physical quantity whose balance is important in geophysical flows [121,122] and in plasma turbulence [123-125]. We argue below that cascade of entropy is a universal feature of turbulence in molecular fluids whose entropy production is at small scales but which are weakly cooled or cooled only at large scales.

\section{A. Cascade of resolved entropy}

We recall that the thermodynamic entropy per volume $s(u, n)$ is a concave function of the internal energy per volume $u$ and the particle number per volume $n$. This follows microscopically from the extensivity of the thermodynamic limit [126,127] and macroscopically from the stability of thermodynamic equilibrium [128]. The entropy is also an analytic function jointly in $u$ and $n$, except at phase transitions, and we restrict our discussion here to single-phase flows. The quantity $s(\bar{u}, \bar{n})$ naturally represents the "large-scale or resolved entropy" for a given length scale $\ell$. We can, in turn, define the "small-scale or unresolved entropy"

$$
\Delta s \equiv \overline{s(u, n)}-s(\bar{u}, \bar{n}) \leq 0 .
$$

The nonpositivity follows by concavity, so spatial coarse graining increases entropy. Throughout our discussion, we use the shorthand notations

$$
\bar{s}=\overline{s(u, n)}, \quad \underline{s}=s(\bar{u}, \bar{n}),
$$

and likewise for other thermodynamic functions of $u, n$. Note that $\langle\underline{s}\rangle$ plays the role of a "cumulative entropy (co) spectrum" up to a wave number of about $1 / \ell$, and $\langle\Delta s\rangle$ is analogous to a second-order "entropy structure function" at separation $\ell$. In many respects, it is more natural to consider a quantity $s_{\max }-s$ that is convex and decreasing in time rather than the traditional entropy, which is instead concave and increasing. The quantity $s_{\max }-s$ was known variously as "capacity of entropy" by Gibbs [129], "deficiency of entropy" by Obukhov [80], and "negentropy" by Brillouin [130]. Here, we use the latter term.

We first derive the balance equation of large-scale entropy at finite $\eta, \zeta, \kappa$ using Eq. (61) for $\bar{u}$ and Eq. (29) for $\bar{\rho}$, with $\overline{\rho \mathbf{v}}=\bar{\rho} \overline{\mathbf{v}}+\bar{\tau}(\rho, \mathbf{v})$. Invoking the first 
law of thermodynamics in the form $d u=T d s+\mu d n$ for absolute temperature $T$ and chemical potential $\mu$, and denoting $\bar{D}_{t}=\partial_{t}+\overline{\mathbf{v}} \cdot \boldsymbol{\nabla}$, we get from

$$
\underline{T} \bar{D}_{t} \underline{s}=\bar{D}_{t} \bar{u}-\underline{\mu} \bar{D}_{t} \bar{n},
$$

after some straightforward calculation, that

$$
\partial_{t} \underline{s}+\boldsymbol{\nabla} \cdot[\underline{s} \overline{\mathbf{v}}+\underline{\beta} \bar{\tau}(u, \mathbf{v})-\underline{\lambda} \bar{\tau}(n, \mathbf{v})]=\Sigma_{\ell}^{\text {inert }},
$$

with inertial-range entropy production given by

$$
\Sigma_{\ell}^{\text {inert }}=-I_{\ell}^{\text {mech }}+\Sigma_{\ell}^{\text {flux }}+\bar{Q}_{\text {visc }} / \underline{T}
$$

for mechanical input of negentropy

$$
I_{\ell}^{\text {mech }}=\frac{\overline{p \Theta}-\underline{p} \bar{\Theta}}{\underline{T}}
$$

and for negentropy flux

$$
\Sigma_{\ell}^{\text {flux }}=\boldsymbol{\nabla} \underline{\beta} \cdot \bar{\tau}(u, \mathbf{v})-\boldsymbol{\nabla} \underline{\lambda} \cdot \bar{\tau}(n, \mathbf{v}) .
$$

The quantity $\beta=1 / T$ in the expressions above is inverse temperature, and $\lambda=\mu / T$ is the thermodynamic potential entropically conjugate to particle number. We now discuss in detail the physical significance of each of these various contributions to the entropy balance.

First, $I_{\ell}^{\text {mech }}$ represents the net input of negentropy [131] into the large scales from pressure work, where $-\underline{p} \bar{\Theta} / \underline{T}$ is the "coherent input" of negentropy at large scales and $\overline{p \Theta} / \underline{T}$ is the entropy production (destruction of negentropy) due to mechanical heating at all scales. There is competition between these two terms but, as anticipated, they will not cancel, in general. We suggest that it is likely that $\langle\underline{p} \bar{\Theta} / \underline{T}\rangle\langle\langle\overline{p \Theta} / \underline{T}\rangle<0$ because of the greater coherence at larger scales and the near cancellations between positive and negative terms at small scales $[104,105]$. An alternative decomposition is

$$
I_{\ell}^{\text {mech }}=\frac{\bar{\tau}(p, \Theta)}{\underline{T}}+I_{\ell}^{\mathrm{flux}}, \quad I_{\ell}^{\mathrm{flux}}=\frac{\Delta p \bar{\Theta}}{\underline{T}}
$$

with $\Delta p=\bar{p}-\underline{p}$. The first term is related to the pressuredilatation defect, and the second term is a "fluxlike" contribution, in the sense that it represents an interaction between a large-scale dilatation $\bar{\Theta}$ and a small-scale pressure $\Delta p$. There is a simple formula for the latter $[90,132]$, which provides an estimate

$$
\Delta p=O\left((\delta u)^{2},(\delta u)(\delta \rho),(\delta \rho)^{2}\right)
$$

yielding a 4/5th-law type representation of the flux term. It is worth noting that for an ideal gas with adiabatic index
$\gamma=c_{P} / c_{V}$, one has $p(u, n)=(\gamma-1) u$ so that $\Delta p=0$ exactly and the fluxlike term is absent. Neither term is present in a naive fine-grained calculation.

Second, $\Sigma_{\ell}^{\text {flux }}$ represents negentropy flux to small scales, arising from small-scale turbulent transport of heat energy $\bar{\tau}(u, \mathbf{v})$ acting against large-scale temperature gradients $\boldsymbol{\nabla} \underline{T}$ and small-scale particle transport $\bar{\tau}(n, \mathbf{v})$ acting against large-scale $\underline{\lambda}$ gradients. These contributions will be positive, indicating entropy production or inverse cascade of entropy or forward cascade of negentropy [133], when the turbulent transport is "down-gradient," with heat transport from higher to lower resolved temperatures $\underline{T}$ and particle transport from higher to lower $\underline{\lambda}$ potential. Because of the lack of scale separation of turbulent transport, both positive and negative values will occur pointwise in spacetime for finite $\ell$, but one should expect that, on average, $\left\langle\Sigma_{\ell}^{\text {flux }}\right\rangle>0$, consistent with the overall increase of entropy from the second law of thermodynamics. Of course, the term $\bar{Q}_{\text {visc }} / \underline{T} \geq 0$ is the resolved entropy production at large scales due to viscous dissipation.

We now consider the situation when there is anomalous entropy production in the ideal limit $\eta, \zeta, \kappa \rightarrow 0$, as hypothesized in Eq. (22). The same result must be obtained by considering either the fine-grained entropy balance (19) or the inertial-range balance (74). Indeed, because of concavity of the volumetric entropy density, the total entropy observed "without spectacles" at resolution $\ell$ can only exceed the true entropy

$$
S_{\ell}(t)=\int d^{d} x s\left(\bar{u}_{\ell}, \bar{\rho}_{\ell}\right) \geq \int d^{d} x s(u, \rho)=S(t),
$$

and in the limit $\ell \rightarrow 0$, they must agree. Thus, if entropy $S(t)$ continues to grow over a finite time interval in the limit $\eta, \zeta, \kappa \rightarrow 0$, then $S_{\ell}(t)$ must also grow for the subsequent limit $\ell \rightarrow 0$ described by a weak Euler solution. Taking the limit $\eta, \zeta, \kappa \rightarrow 0$ of the fine-grained entropy balance (19), the anomaly is represented as

$$
\partial_{t} s+\nabla \cdot(s \mathbf{v})=\Sigma^{\text {diss }}
$$

with $\Sigma^{\text {diss }}=\Sigma^{\text {therm }}+\Sigma^{\text {visc }}$, for viscous entropy production

$$
\Sigma^{\mathrm{visc}}=\beta * Q_{\mathrm{visc}}=\mathcal{D}-\lim _{\eta, \zeta, \kappa \rightarrow 0} \beta Q_{\mathrm{visc}}
$$

and for entropy production by thermal conduction

$$
\Sigma^{\text {therm }}=\mathcal{D}-\lim _{\eta, \zeta, \kappa \rightarrow 0} \frac{\kappa|\nabla T|^{2}}{T^{2}} .
$$

The coarse-grained entropy balance (74) in the limit $\eta, \zeta$, $\kappa \rightarrow 0$ is unchanged, except that $\overline{p \Theta} \rightarrow \overline{p * \Theta}$. In the subsequent limit $\ell \rightarrow 0$, the inertial-range entropy balance becomes 


$$
\partial_{t} s+\boldsymbol{\nabla} \cdot(s \mathbf{v})=\Sigma^{\text {inert }},
$$

where $\Sigma^{\text {inert }}=-I^{\text {mech }}+\Sigma^{\text {flux }}+\beta \circ Q_{\text {visc }}$ with [134]

$$
\beta \circ Q_{\mathrm{visc}}=\mathcal{D}-\lim _{\ell \rightarrow 0} \underline{\beta} \bar{Q}_{\mathrm{visc}}
$$

and where $I^{\text {mech }}=I_{\text {flux }}+\beta \circ \tau(p, \Theta)$ with

$$
\beta \circ \tau(p, \Theta)=\mathcal{D}-\lim _{\ell \rightarrow 0} \underline{\beta} \bar{\tau}(p, \Theta) .
$$

The expressions (84)-(86) provide an inertial-range representation of anomalous entropy production. Equating the two different expressions, $\Sigma_{\text {inert }}=\Sigma_{\text {diss }}$.

\section{B. Singularity conditions and intrinsic entropy}

The above general results are nicely illustrated by planar shocks in an ideal gas for the special value of Prandtl number $\operatorname{Pr}=3 / 4$. There is, in fact, a two-parameter family of stationary shocks in ideal gases, labeled by the adiabatic index $\gamma>1$ and by the preshock Mach number $M_{0}>1$, or, alternatively, the compression ratio $R=(\gamma+1) /[(\gamma-1)+$ $\left.2 / M_{0}^{2}\right]$. All of the anomalous quantities in the balances above are nonzero for $\operatorname{Pr}=3 / 4$ (with the exception of $I_{\text {flux }}$ since $\Delta p \equiv 0$ for an ideal gas) and are proportional to Dirac delta functions at the location of the shock. For a shock situated at the origin, there is a positive entropy production anomaly of the simple form

$$
\Sigma_{\text {inert }}=\Sigma_{\text {diss }}=\left(\Delta s_{m}\right) j_{*} \delta(x),
$$

where $\Delta s_{m}=s_{m, 1}-s_{m, 0}>0$ is the jump across the shock of the entropy per mass $s_{m}=s / \rho$ (with " 0 " denoting the gas in front of the shock and "1" the gas behind the shock) and $j_{*}=j_{0}=j_{1}>0$ is the mass flux through the shock. Explicit expressions for all terms in the entropy balance are given in Appendix A. As expected, the inertial-range quantities $\Sigma_{\text {flux }}$ and $\beta \circ\left(Q_{\text {visc }}-\tau(p, \Theta)\right)$ in the infinite Reynolds-number limit are identical for all planar, ideal-gas shocks with the same values of $\gamma$ and $M_{0}$, entirely independent of the precise molecular dissipation mechanism. In particular, these two specific quantities are Prandtl-number independent. It is interesting that the negentropy flux is nonzero for such shocks, even though the energy flux vanishes exactly. This clearly shows that the two cascades are distinct, in general. In fact, it is the negentropy flux $\Sigma_{\text {flux }}$ that, inside the inertial-range of planar, ideal-gas shocks, supplies the contribution $\left(\Delta s_{m}\right) j_{*} \delta(x)$, arising from the particle transport term $-\boldsymbol{\nabla} \underline{\lambda} \cdot \bar{\tau}(n, \mathbf{v})$. All other inertial-range contributions cancel between $I_{\text {mech }}, \Sigma_{\text {flux }}$, and $\beta \circ Q_{\text {visc }}$. See Appendix A for details.

While Euler shock solutions with discontinuous fields provide a simple example where the negentropy flux is nonvanishing, more modest singularities are able to support a negentropy cascade. Note that, from formula (79) for $\Delta p$ and the definition (78) of $I_{\ell}^{\text {flux }}$,

$$
I_{\ell}^{\mathrm{flux}}=O\left((\delta u)^{2},(\delta u)(\delta \rho),(\delta \rho)^{2}\right) O\left(\frac{\delta v}{\ell}\right) .
$$

Hence, this term may have a nonvanishing limit as $\ell \rightarrow 0$ whenever

$$
2 \min \left\{\zeta_{q}^{u}, \zeta_{q}^{\rho}\right\}+\zeta_{q}^{v} \leq q, \quad q \geq 3 .
$$

Likewise, from the chain rule for gradients of the smooth functions $\beta(u, \rho)$ and $\lambda(u, \rho)$, one gets

$$
\boldsymbol{\nabla} \underline{\beta}, \boldsymbol{\nabla} \underline{\lambda}=\frac{O(\delta u, \delta \rho)}{\ell},
$$

which, with the general result $\tau(f, g)=O(\delta f \cdot \delta g)$ and the definition of $\Sigma_{\ell}^{\text {flux }}$, gives the identical estimate

$$
\Sigma_{\ell}^{\mathrm{flux}}=O\left((\delta u)^{2},(\delta u)(\delta \rho),(\delta \rho)^{2}\right) O\left(\frac{\delta v}{\ell}\right) .
$$

Thus, the inequality (89) again provides a necessary condition for a nonvanishing limit as $\ell \rightarrow 0$. The shock solutions with discontinuous fields have $\zeta_{q}^{u}=\zeta_{q}^{\rho}=\zeta_{q}^{v}=1$ for $q \geq 1$ and thus satisfy these inequalities for all $q \geq 3$. However, multifractal fields $u, \rho, \mathbf{v}$ with positive Hölder exponents can also easily satisfy these inequalities [37]. Thus, for compressible turbulent flow, the anomalous entropy production should generally arise not just from shocks with zero Hölder exponents but also from the spectrum of milder Hölder singularities.

When the singularity conditions (51) and (53) are not satisfied, then one expects that entropy will in fact be conserved. This statement is an analogue of the Onsager singularity theorem for a dissipative anomaly of negentropy. Such a result does not follow directly from the estimates (88) and (91) on the fluxes because of the additional terms contributing to the inertial-range entropy balance. However, such a result may be proved [90] by the following arguments. First, rewrite the inertial-range entropy balance as

$$
\begin{aligned}
& \partial_{t} \underline{\underline{s}}+\boldsymbol{\nabla} \cdot[\underline{s} \overline{\mathbf{v}}+\underline{\beta} \bar{\tau}(u, \mathbf{v})-\underline{\lambda} \bar{\tau}(n, \mathbf{v})] \\
& \quad=\Sigma_{\ell}^{\mathrm{flux}}-I_{\ell}^{\mathrm{flux}}+\underline{\beta}\left(\bar{Q}_{\mathrm{visc}}-\bar{\tau}(p, \Theta)\right) .
\end{aligned}
$$

The first two terms on the right are those that have been shown to vanish as $\ell \rightarrow 0$ when Eq. (89) is not satisfied. To evaluate the last term, we use Eq. (66) for the subscale kinetic energy. The first two terms on the right of Eq. (66) are exactly those appearing in the entropy balance, while the third is the energy flux. Thus, multiplying Eq. (66) by $\underline{\beta}$ gives 


$$
\begin{aligned}
\underline{\beta}\left(\bar{Q}_{\text {visc }}-\bar{\tau}(p, \Theta)\right) \\
=\underline{\beta}_{\ell}^{\text {flux }}+\left(\partial_{t} \underline{\beta}\right) \frac{1}{2} \bar{\rho} \tilde{\tau}\left(v_{i}, v_{i}\right) \\
\quad+\boldsymbol{\nabla} \underline{\beta} \cdot\left[\frac{1}{2} \bar{\rho} \tilde{\tau}\left(v_{i}, v_{i}\right) \tilde{\mathbf{v}}+\bar{\tau}(p, \mathbf{v})+\frac{1}{2} \bar{\rho} \tilde{\tau}\left(v_{i}, v_{i}, \mathbf{v}\right)\right] \\
\quad+(\cdots),
\end{aligned}
$$

where $(\cdots)$ denotes a total derivative term that vanishes distributionally in the limit $\ell \rightarrow 0$. The other three terms are all fluxlike. The first of these contains a time derivative, which is perhaps unexpected, but the physical meaning is clearly an entropy production due to the rate of change of large-scale inverse temperature times subscale kinetic energy. The term $\beta Q_{\ell}^{\text {flux }}$ is an entropy production due to kinetic-energy cascade, and the terms proportional to $\boldsymbol{\nabla} \underline{\beta}$ are a correction to the turbulent internal-energy transport $\bar{\tau}(u, \mathbf{v})$. These flux terms vanish as explicit power laws in the limit $\ell \rightarrow 0$ for solutions that are not sufficiently singular. Precisely, at least one of the following conditions must be satisfied:

$$
\begin{gathered}
2 \min \left\{\zeta_{q}^{u}, \zeta_{q}^{\rho}\right\}+\zeta_{q}^{v} \leq q \quad q \geq 3, \\
\min \left\{\zeta_{q}^{u}, \zeta_{q}^{\rho}\right\}+2 \zeta_{q}^{v} \leq q \quad q \geq 3, \\
3 \zeta_{q}^{v} \leq q \quad q \geq 3,
\end{gathered}
$$

for the fluxes not to vanish. Here, Eq. (94) is the same as Eq. (89), and Eq. (53) has also been replaced by Eq. (94), which implies vanishing of baropycnal work via Eq. (52).

There is, in fact, a much more fundamental way to reach the same conclusion. Let us define an intrinsic large-scale or resolved entropy density by

$$
\underline{s}^{*}=\underline{s}+\frac{1}{2} \underline{\beta} \bar{\rho} \tilde{\tau}\left(v_{i}, v_{i}\right) .
$$

Clearly, $\underline{s}^{*} \geq \bar{s}$. From the homogeneous Gibbs relation $\underline{s}=\underline{\beta}(\bar{u}+\underline{p})-\underline{\lambda} \bar{n}$, it follows that $\underline{s}^{*}=\underline{\beta}\left(\bar{u}^{*}+\underline{p}\right)-\underline{\lambda} \bar{n}$, where $\bar{u}^{*}$ is the intrinsic large-scale internal energy that was introduced in Eq. (64). Using Eq. (63) for the intrinsic internal energy, the coarse-grained mass conservation equation (29), and the standard thermodynamic relation $d(\underline{\beta} \underline{p})=\bar{n} d \underline{\lambda}-\bar{u} d \underline{\beta}$, it is then straightforward to verify the entropy balance equation

$$
\partial_{t} \underline{s}^{*}+\nabla \cdot \underline{\mathbf{s}}^{*}=\Sigma_{\ell}^{\text {inert* }}
$$

where

$$
\begin{aligned}
\underline{\mathbf{s}}^{*}= & \underline{s} \overline{\mathbf{v}}+\underline{\beta} \bar{\tau}(u, \mathbf{v})-\underline{\lambda} \bar{\tau}(n, \mathbf{v}) \\
& +\underline{\beta}\left[\frac{1}{2} \bar{\rho} \tilde{\tau}\left(v_{i}, v_{i}\right) \tilde{\mathbf{v}}+\frac{1}{2} \bar{\rho} \tilde{\tau}\left(v_{i}, v_{i}, \mathbf{v}\right)+\bar{\tau}(p, \mathbf{v})\right]
\end{aligned}
$$

is the spatial current of intrinsic entropy and where $\Sigma_{\ell}^{\text {inert* }}=-I_{\ell}^{\text {flux }}+\Sigma_{\ell}^{\text {flux* }}$, with

$$
\begin{aligned}
\Sigma_{\ell}^{\text {flux } *}= & \Sigma_{\ell}^{\text {flux }}+\underline{\beta} Q_{\ell}^{\text {flux }}+\left(\partial_{t} \underline{\beta}\right) \frac{1}{2} \bar{\rho} \tilde{\tau}\left(v_{i}, v_{i}\right) \\
& +\boldsymbol{\nabla} \underline{\beta} \cdot\left[\frac{1}{2} \bar{\rho} \tilde{\tau}\left(v_{i}, v_{i}\right) \tilde{\mathbf{v}}+\frac{1}{2} \bar{\rho} \tilde{\tau}\left(v_{i}, v_{i}, \mathbf{v}\right)+\bar{\tau}(p, \mathbf{v})\right] \\
= & \boldsymbol{\nabla} \underline{\beta} \cdot\left[\bar{\tau}(h, \mathbf{v})+\frac{1}{2} \bar{\rho} \tilde{\tau}\left(v_{i}, v_{i}, \mathbf{v}\right)\right] \\
& -\boldsymbol{\nabla} \underline{\lambda} \cdot \bar{\tau}(n, \mathbf{v})+\underline{\beta} Q_{\ell}^{\text {flux }}+\left(\tilde{D}_{t} \beta\right) \frac{1}{2} \bar{\rho} \tilde{\tau}\left(v_{i}, v_{i}\right)
\end{aligned}
$$

the flux of intrinsic inertial-range negentropy. Although this result is the same as that obtained by substituting Eq. (93) into Eq. (92), the present derivation is more general because it makes no reference to any microscopic model. Thus, Eq. (98) is seen to be valid for all distributional Euler solutions, including those derived from Boltzmann kinetic theory [63], for example, and not restricted to limits of compressible Navier-Stokes solutions.

Taking the limit as $\ell \rightarrow 0$ of the inertial-range balance equation (98) yields again the limiting balance (84) for the distributional solution of the compressible Euler equations. One concludes that any solution that is too regular, satisfying none of the conditions (94)-(96), will obey local entropy conservation:

$$
\partial_{t} s+\boldsymbol{\nabla} \cdot(s \mathbf{v})=0
$$

Put another way, $\Sigma_{\text {diss }}=0$ unless the fluid fields possess singularities compatible with (94)-(96). For details of the proof, see the companion paper [90]. These arguments also allow one to complete the proof that energy dissipation anomalies must vanish when (94)-(96) are not satisfied. Because of the non-negativity of the separate viscous and thermal conduction contributions to anomalous entropy production, $\Sigma_{\text {diss }}=0$ immediately implies that

$$
\Sigma_{\text {therm }}=0, \quad \Sigma_{\text {visc }}=\beta * Q_{\text {visc }}=0 .
$$

It is not hard to see that the second condition implies that $Q_{\text {visc }}=0$ since $\beta \circ Q_{\text {visc }} \geq Q_{\text {visc }} /\|T\|_{\infty} \geq 0$ [90]. Coupled with $Q_{\text {flux }}=0$, we obtain from Eq. (56) that $\tau(p, \Theta)=0$ or $p \circ \Theta=p * \Theta$. Thus, kinetic-energy balance (46) becomes

$$
\partial_{t}\left(\frac{1}{2} \rho v^{2}\right)+\boldsymbol{\nabla} \cdot\left[\left(p+\frac{1}{2} \rho v^{2}\right) \mathbf{v}\right]=p * \Theta,
$$

and internal-energy balance (65) becomes

$$
\partial_{t} u+\boldsymbol{\nabla} \cdot(u \mathbf{v})=-p * \Theta .
$$

In other words, kinetic-energy and internal-energy balances hold without anomalies for flows more regular than allowed 
by (94)-(96). It is interesting that the proof of a complete Onsager singularity theorem for energy dissipation anomalies in compressible turbulence seems to require an essential consideration of entropy.

\section{Steady-state entropy balances}

We can now discuss entropy balances in steadystate turbulence of a compressible fluid, which is forced mechanically and also cooled, e.g., by radiation, governed by Eqs. (68)-(70). It is easy to see that Eq. (63) for intrinsic resolved internal energy is modified by the addition of two terms:

$$
\partial_{t} \bar{u}^{*}=\cdots+\bar{\rho} \tilde{\tau}\left(\mathbf{v} ; \mathbf{a}_{\mathrm{ext}}\right)-\bar{Q}_{\mathrm{cool}} \cdot
$$

The first term $\bar{\rho} \tilde{\tau}\left(\mathbf{v} ; \mathbf{a}_{\text {ext }}\right)$ is negligible when $\ell \ll L$, where $L$ is the length scale of the smooth acceleration field $\mathbf{a}_{\mathrm{ext}}$. However, the second term $-\bar{Q}_{\text {cool }}$ has a nonzero limit as $\ell \rightarrow 0$ and contributes to the balance (98) of intrinsic resolved entropy an additional term $-\underline{\beta} \bar{Q}_{\text {cool }}$ on the right, which represents the removal of entropy at large scales by cooling. Likewise, the fine-grained entropy balance (19) gains an additional term $-\beta Q_{\text {cool }}$ on the right. A statistically stationary and homogeneous steady state thus must satisfy

$$
\left\langle\Sigma^{\text {diss }}\right\rangle=\left\langle\beta Q^{\text {cool }}\right\rangle
$$

from the fine-grained balance and

$$
\begin{aligned}
\left\langle\Sigma_{\ell}^{\text {inert* }}\right\rangle & =\left\langle\underline{\beta}_{\ell} \bar{Q}_{\ell}^{\text {cool }}\right\rangle+\left\langle\underline{\beta}_{\ell} \bar{\rho}_{\ell} \tilde{\tau}_{\ell}\left(\mathbf{v} ; \mathbf{a}_{\mathrm{ext}}\right)\right\rangle \\
& \approx\left\langle\beta Q^{\text {cool }}\right\rangle, \quad \ell \ll L
\end{aligned}
$$

from the coarse-grained or inertial-range balance [135]. The physical picture is that the large-scale negentropy introduced by cooling cascades through an inertial range down to small scales where it is canceled by microscopic entropy production. Alternatively, we may write Eq. (107) as

$$
\begin{aligned}
\left\langle\Sigma_{\ell}^{\text {flux* }}\right\rangle & =\left\langle I_{\ell}^{\text {flux }}\right\rangle+\left\langle\underline{\beta}_{\ell} \bar{Q}_{\ell}^{\text {cool }}\right\rangle+\left\langle\underline{\beta}_{\ell} \bar{\rho}_{\ell} \tilde{\tau}_{\ell}\left(\mathbf{v} ; \mathbf{a}_{\text {ext }}\right)\right\rangle \\
& \approx\left\langle I^{\text {flux }}\right\rangle+\left\langle\beta Q^{\text {cool }}\right\rangle, \quad \ell \ll L
\end{aligned}
$$

so that the flux of intrinsic entropy through the initial range also includes negentropy input from anomalous pressure work, as well as from large-scale cooling. For an ideal-gas equation of state, $I_{\ell}^{\text {flux }}=0$, of course, and $\left\langle\Sigma_{\ell}^{\text {flux* }}\right\rangle \approx\left\langle\Sigma^{\text {diss }}\right\rangle$ for $\ell \ll L$.

In addition to these exact relations, some approximate balances are suggested by our results. For the purpose of a qualitative picture, let us assume an approximately constant temperature $T$. This gives (on average) the fine-grained (dissipation-range) entropy balance

$$
\left\langle\Sigma_{\text {therm }}\right\rangle+\frac{\langle Q\rangle}{T}=\frac{\left\langle Q_{\text {cool }}\right\rangle}{T}
$$

and from Eq. (75) the coarse-grained (inertial-range) entropy balance

$$
\left\langle\Sigma_{\text {flux }}\right\rangle+\frac{\langle Q\rangle}{T}=\frac{\left\langle Q_{\text {cool }}\right\rangle}{T}+\left\langle I_{\text {mech }}\right\rangle .
$$

One also has the mean energy balance $\left\langle Q_{\text {cool }}\right\rangle=\langle Q\rangle+$ $\left\langle Q_{\text {trans }}\right\rangle$ with $Q_{\text {trans }}=-p * \Theta$. Note that the fine-grained pressure work $Q_{\text {trans }}$ is an incoherent transfer of energy, which might be better denoted in this context as $Q_{\text {incoh }}$. Equation (109) thus gives

$$
\left\langle\Sigma_{\text {therm }}\right\rangle=\frac{\left\langle Q_{\text {incoh }}\right\rangle}{T} .
$$

Decomposing $\left\langle I_{\text {mech }}\right\rangle=\left(\left\langle Q_{\text {coh }}\right\rangle-\left\langle Q_{\text {incoh }}\right\rangle\right) / T$ with the definition $\left\langle Q_{c o h}\right\rangle=-\lim _{\ell \rightarrow 0}\langle\underline{p} \bar{\Theta}\rangle$ of coherent work input, substituting into Eq. (110), and using $\left\langle Q_{\text {cool }}\right\rangle=\langle Q\rangle+$ $\left\langle Q_{\text {incoh }}\right\rangle$ again yields

$$
\left\langle\Sigma_{\text {flux }}\right\rangle=\frac{\left\langle Q_{\text {coh }}\right\rangle}{T} .
$$

The above relations implicitly assume that $\left\langle\beta * Q_{\text {visc }}\right\rangle \simeq$ $\left\langle\beta \circ Q_{\text {visc }}\right\rangle \simeq\langle Q\rangle / T$. This heuristic argument suggests that the coherent input of negentropy at large scales by anomalous pressure work will be cascaded downscale, while the positive entropy input due to fine-grained (incoherent) transfer from mechanical to internal energy will go into entropy production due to thermal conduction. Needless to say, this is a nonrigorous mean-field argument ignoring temperature fluctuations and must be subject to empirical tests in order to determine its range of validity. It clearly requires a sufficiently large Mach number since $Q_{\text {coh }}, Q_{\text {incoh }}$ both vanish for incompressible flow. However, like our earlier arguments, it supports the conclusion that compressible turbulence will generally involve a forward cascade of negentropy or, equivalently, an inverse cascade of the traditional entropy.

\section{Incompressible limit}

All of our analysis of entropy cascade can be carried out for the special case of the incompressible fluid equations (20) and (21) that were derived assuming balanced mechanical and thermal forcing, with $u=\rho c_{P} T$, $s=\rho c_{P} \ln T$, and with chemical potential per mass $\mu=c_{P} T-c_{P} T \ln T$, so that the Gibbs relation $T s=u-$ $\mu \rho$ is satisfied. In the same manner as for compressible fluids, we can derive from Eqs. (20) and (21) the following balance equation for resolved/large-scale entropy:

$$
\begin{aligned}
\partial_{t} \underline{s} & +\boldsymbol{\nabla} \cdot[\underline{s} \overline{\mathbf{v}}+\underline{\beta} \bar{\tau}(u, \mathbf{v})-\chi \boldsymbol{\nabla} \underline{s}] \\
& =\boldsymbol{\nabla} \underline{\beta} \cdot \bar{\tau}(u, \mathbf{v})+\kappa|\boldsymbol{\nabla} \bar{T}|^{2} / \bar{T}^{2}+\bar{Q}_{\text {visc }} / \bar{T},
\end{aligned}
$$

with $\chi=\kappa / \rho c_{P}$ the thermal diffusivity. In the limit $\kappa, \eta \rightarrow 0$ with $\ell$ fixed, the terms involving $\kappa$ disappear, but the term 
involving viscous energy dissipation $\bar{Q}_{\text {visc }}$ remains. Equation (113) in that limit may be formally obtained directly from the general result (92) by setting $\Theta=0$ and $n=$ (const). The negentropy flux for an incompressible fluid is given by the first term on the right-hand side of Eq. (113), or

$$
\Sigma_{\ell}^{\mathrm{flux}}=\boldsymbol{\nabla} \underline{\beta} \cdot \bar{\tau}(u, \mathbf{v})=O\left(\frac{(\delta T)^{2}(\delta v)}{\ell}\right) .
$$

In the balance equation (113) for entropy, we generalize the one of Obukhov [80] in two ways: (i) We keep the contribution from $\bar{Q}_{\text {visc }}$ that he neglected [136], and (ii) we do not approximate $s \simeq \rho c_{P} \ln T_{0}+(1 / 2) \rho c_{P}\left(T^{\prime} / T_{0}\right)^{2}$ by assuming small fluctuations $T^{\prime}$ around background temperature $T_{0}$. By applying a point-splitting argument to the latter quadratic approximation, Yaglom [137] derived an expression for negentropy flux in terms of velocity and temperature increments, analogous to the 4/5th law of Kolmogorov. Our expression for negentropy flux (114) generalizes that relation to the exact entropy.

The intrinsic resolved entropy may also be defined for an incompressible fluid as $\underline{s}^{*}=\underline{s}+\underline{\beta}(1 / 2) \rho \bar{\tau}\left(v_{i}, v_{i}\right)$. From the coarse-grained versions of the ideal incompressible equations (20) and (21) for $\eta, \kappa \rightarrow 0$, the balance equation of the intrinsic entropy may be derived as

$$
\begin{aligned}
\partial_{t} \underline{s}^{*} & +\boldsymbol{\nabla} \cdot\left[\underline{s}^{*} \overline{\mathbf{v}}+\underline{\beta} \bar{\tau}(p, \mathbf{v})+\rho(\underline{\beta} / 2) \bar{\tau}\left(v_{i}, v_{i}, \mathbf{v}\right)\right] \\
= & \underline{\beta} Q_{\ell}^{\text {flux }}+\left(\bar{D}_{t} \underline{\beta}\right)(1 / 2) \rho \bar{\tau}\left(v_{i}, v_{i}\right) \\
& +\boldsymbol{\nabla} \underline{\beta} \cdot\left[\bar{\tau}(h, \mathbf{v})+(1 / 2) \rho \bar{\tau}\left(v_{i}, v_{i}, \mathbf{v}\right)\right]
\end{aligned}
$$

with $h=u+p$. Unlike the balance equation (113) for the (naive) resolved entropy $\underline{s}$ as $\eta, \kappa \rightarrow 0$, the balance equation (115) for intrinsic entropy $\underline{s}^{*}$ does not contain any terms involving $\eta, \kappa$ and can be evaluated purely from coarse-grained measurements of the basic fluid fields $\mathbf{v}, T$ and their products. Equation (115) is the incompressible analogue of Eqs. (98)-(100) for compressible fluids and can be formally obtained from them by setting $\Theta=0$ and $n=$ (const). Whenever there is anomalous entropy production, there must be an inertial-range flux of intrinsic negentropy, with some of the terms on the right-hand side of Eq. (115) nonvanishing as $\ell \rightarrow 0$. This follows by the same argument as for the compressible fluid: If finegrained entropy $S(t)=\int d^{d} x s$ is increasing in time, then any coarse-grained measurements at resolution $\ell$ must show a similar increase in $\underline{S}^{*}(t)=\int d^{d} x \underline{s}^{*}$ since $\underline{s}^{*} \geq \bar{s}$.

Exact steady-state entropy balances for an incompressible fluid that is stirred and cooled only at large scales can be derived similarly to Eqs. (106)-(108) for the compressible fluid. This is the same problem considered by Refs. [80,137], and we modestly generalize their results for this problem. Since there is no anomalous input $I_{\ell}^{\text {flux }}$ for incompressible flow, the inertial-range balance for incompressible turbulence analogous to Eq. (108) becomes

$$
\left\langle\Sigma_{\ell}^{\text {flux* }}\right\rangle \approx\left\langle\beta Q^{\text {cool }}\right\rangle, \quad \ell \ll L,
$$

with $\Sigma_{\ell}^{\text {flux* }}$ defined by the right-hand side of Eq. (115). The approximate balances (111) and (112) reduce for incompressible flow to the prediction that $\left\langle\Sigma^{\text {therm }}\right\rangle=\left\langle\Sigma_{\ell}^{\text {flux }}\right\rangle=0$. However, these are "mean-field relations" assuming a nearly constant temperature field, and they are especially unlikely to hold in the incompressible limit, when $u$ and $s$ vary only because of temperature.

In addition to generalizing the classical theories for a fluid that is stirred and heated or cooled at large scales [80,137], our analysis also predicts an inertial-range negentropy cascade whenever there is anomalous entropy production at small scales and negligible cooling. An example is freely decaying incompressible fluid turbulence with an initial uniform temperature $T_{0}$. This is not a situation where negentropy cascade is predicted by the classical theories, but our analysis implies that $\left\langle\Sigma_{\ell}^{\text {flux* }}\right\rangle \approx\left\langle\Sigma^{\text {diss }}\right\rangle$ for $\ell \ll L$. The microscopic entropy production $\Sigma^{\text {diss }}$ is expected to be nonvanishing at least because of the contribution $\beta Q_{\text {visc }}$ from viscous heating. In this situation, the temperature $T$ will, on average, slowly increase in time because of the viscous heating, but it may be expected that $\underline{\beta}$ remains approximately constant in space at inertial-range lengths $\ell$. The mean-field relations (111) and (112) should hold quite well, predicting that $\left\langle\Sigma^{\text {therm }}\right\rangle=\left\langle\Sigma_{\ell}^{\text {flux }}\right\rangle=0$. The most likely source of negentropy cascade is the first term $\beta Q_{\ell}^{\text {flux }}$ in $\Sigma_{\ell}^{\text {flux* }}$, which would imply that entropy flux $\left\langle\Sigma_{\ell}^{\text {flux* }}\right\rangle \approx\left\langle Q_{\ell}^{\text {flux }}\right\rangle / T$, completely enslaved to the incompressible energy cascade. However, it is also possible that the second term in Eq. (115) proportional to $\bar{D}_{t} \underline{\beta}$ may contribute non-negligibly because of the secular increase in temperature. Empirical investigation will be required to clarify the relative magnitudes of the various contributions to $\left\langle\Sigma_{\ell}^{\text {flux } *}\right\rangle$.

\section{RELATIONS TO OTHER APPROACHES}

We now briefly discuss the relation of our analysis with other approaches to compressible fluid turbulence that have been proposed.

\section{A. Barotropic models}

Barotropicity is a common assumption that is employed to simplify the description of compressible turbulence, with pressure $p=p(\rho)$ taken to be a function of mass density only. Polytropic models with $p(\rho)=K \rho^{\gamma}$ are a special case. This assumption has been employed in many classical theoretical papers $[50,51]$ and also in more recent theoretical works $[54,55,138]$. Barotropic models are often also employed for numerical simulations, especially in astrophysical applications $[139,140]$. There is no explicit representation of thermodynamic entropy in such models, and thus no entropy cascade of the type considered in this paper can occur there. Such simulations nevertheless 
exhibit a kind of compressible turbulence and therefore might be taken as counterexamples to our predicted entropy cascade. This is only the case, however, if barotropic models can be valid descriptions of compressible turbulence in a molecular fluid that is weakly cooled or cooled only at large scales, which is the proposed domain of our theory. This is a serious open question. Unlike the compressible Navier-Stokes equation, which can be systematically derived from the Boltzmann equation or even Hamiltonian molecular dynamics in the limit of small Knudsen number [141-143], there seems to be no similar derivation of barotropic fluid models from first principles. One must therefore carefully consider whether these models can be valid descriptions of molecular fluids not interacting strongly with other systems.

Ideal barotropic models can be understood to describe molecular fluids in a very simple way. No explicit equation for internal energy is solved in such models, and instead, the internal energy per mass is obtained from

$$
e(\rho)=\int p(\rho) \frac{d \rho}{\rho^{2}} .
$$

Thus, $D_{t} \rho=-\rho(\boldsymbol{\nabla} \cdot \mathbf{v})$ implies $\rho D_{t} e=-p(\boldsymbol{\nabla} \cdot \mathbf{v})$, and then $u=\rho e$ is recovered, satisfying

$$
\partial_{t} u+\boldsymbol{\nabla} \cdot(u \mathbf{v})=-p(\boldsymbol{\nabla} \cdot \mathbf{v}) .
$$

The first law of thermodynamics in the form

$$
d e=T d s_{m}+\frac{p}{\rho^{2}} d \rho,
$$

with $s_{m}=s / \rho$ the entropy per mass, shows that $u$ obtained as above can consistently represent internal energy only when either $T=0$ (so that $s=0$ by the Nernst law) or entropy per particle $s_{n}=s / n$ is constant. For a smooth solution of the ideal Euler equations, the entropy is a locally conserved quantity, as can be seen by setting $\eta=\zeta=\kappa=$ 0 in Eq. (19), so an initially isentropic flow remains isentropic. We thus recover a well-known interpretation of ideal barotropic models as a description of isentropic, laminar flows with negligible molecular dissipation.

Barotropic fluid models with viscous stresses added to the momentum equation are much more problematic [144]. The interpretation of the quantity $e$ given by Eq. (117) as internal energy per mass leads to an obvious contradiction with conservation of total fluid energy since, according to Eq. (118), the energy lost by viscous dissipation of kinetic energy does not reappear as increased internal energy of the fluid. It has been suggested to us by two anonymous referees that the integral in Eq. (117) should instead represent only the "work part" $w=-\int p d(1 / \rho)$ of $e$ and that conservation of energy is maintained by transfer of "barotropic energy" $E_{B}=(1 / 2) \rho v^{2}+\rho w$ to the "heat part" $q=\int T d s_{m}$, not explicitly represented in the model.
In this view, the viscous barotropic model is a reduced description of the molecular fluid, similar to the incompressible Navier-Stokes equation (20), which also dissipates kinetic energy but for which the total fluid energy $E=(1 / 2) \rho v^{2}+\rho c_{P} T$ is conserved. In that analogy, a full description of a barotropic fluid requires an additional dynamical equation for $s_{m}$ (or other thermodynamic variable), similar to Eq. (21) for the temperature field of an incompressible fluid. There seems to be no study that derives viscous barotropic models in this fashion, and it is not clear what physical parameter would determine the validity of such a reduced description, analogous to Mach number for an incompressible fluid. However, if correct, this would be one possible means to justify viscous barotropic models as a valid description of a molecular fluid, consistent with equilibrium thermodynamics, conservation of energy, and the nonequilibrium second law.

There are substantial arguments against this view, in our opinion. First, the work part $w$ and heat part $q$ are not thermodynamic state variables because $₫ q=T d s_{m}$, đ $w=$ $-p d(1 / \rho)$ are not exact differentials and, as is well known, their integrals depend upon the path in state space $\left(\rho, s_{m}\right)$. Without specifying a particular integration path, the "barotropic energy" $E_{B}=(1 / 2) \rho v^{2}+\rho w$ is ambiguous, and no comparison with laboratory experiment or a more detailed model (such as compressible Navier-Stokes) is possible. This is quite different from the incompressible limit, where $u$ and $T$ are well-defined thermodynamic state variables for flows at any Mach number. An even more serious issue is that it is quite difficult to see how a barotropic model for pressure $p=p_{B}(\rho)$ can be consistent with the exact thermodynamic pressure $p=p\left(\rho, s_{m}\right)$, when entropy per mass $s_{m}$ is increasing along fluid particle trajectories. Even if the two expressions for pressure agree at an initial time, the growth of $s_{m}$ will lead to their disagreement at later times. In Appendix B, we present an exact no-go theorem of this type, proving that there is no sequence of compressible flows $\left(\mathbf{v}^{\epsilon}, \rho^{\epsilon}, s_{m}^{\epsilon}\right)$ for which the barotropic closure can become asymptotically exact, with $p\left(\rho^{\epsilon}, s_{m}^{\epsilon}\right)-p_{B}\left(\rho^{\epsilon}\right) \rightarrow 0$ as some parameter $\epsilon \rightarrow 0$, unless the entropy production per volume $\Sigma^{\epsilon}$ tends to zero everywhere in the same limit. For these reasons, we believe that viscous barotropic models have questionable validity as descriptions of dissipative molecular fluids evolving as nearly isolated systems (e.g., not cooled by effective coupling with radiation). Barotropic models could, of course, potentially be valid descriptions of fluid systems in other situations than these.

Therefore, we cannot immediately accept barotropic models as physical counterexamples to our prediction of an entropy cascade in turbulence of molecular fluids that are weakly cooled or cooled only at large scales. The preceding no-go argument against barotropic closures applies even in the ideal limit as $\eta, \zeta, \kappa \rightarrow 0$, if turbulent entropy production is anomalous and persists in the limit. 
The inconsistency of the barotropic closure with viscous entropy production due to shocks has been discussed previously in the mathematics literature, e.g. Ref. [82], pp. 13-14. In addition to the above theoretical problems, it is also observed empirically that the isentropic approximation is not well satisfied pointwise in subsonic and transonic turbulence for an ideal gas $[105,145]$. Substantial entropy fluctuations $s_{m}=c_{V} \log \left(p / K \rho^{\gamma}\right)$ of the ideal gas are found, which are completely neglected by barotropic models and are physically significant. It is possible, in principle, that viscous barotropic models may approximate some features of turbulence in molecular fluids, e.g., velocity statistics, even though the barotropic closure for pressure is not consistent with all physical principles. Viscous barotropic models certainly fail to represent the entropy cascade derived in this work from the full compressible Navier-Stokes equation, but we argue that this is a failure of the barotropic models and not of our prediction.

The polytropic model $p=K \rho$ requires special discussion because it is often interpreted by means of the idealgas law $p=n k_{B} T_{0}$ as an isothermal ideal gas, very strongly cooled to a uniform temperature $T_{0}$ at all length scales, rather than as an isentropic fluid. This is a good example of a barotropic model that arises as a valid description of a molecular fluid but in a limit of extreme coupling to radiation. The temperature equation can be easily checked for a general equation of state to have the form

$$
\rho c_{V} D_{t} T=-T\left(\frac{\partial p}{\partial T}\right)_{n} \Theta+\nabla \cdot(\kappa \nabla T)+Q_{\text {visc }}
$$

where $c_{V}(n, T)$ and $p(n, T)$ are taken to be functions of the two independent thermodynamic variables $n, T$. For an ideal gas, this simplifies further to

$$
\rho c_{V} D_{t} T=-p \Theta+\nabla \cdot(\kappa \nabla T)+Q_{\text {visc }},
$$

with $c_{V}=\alpha k_{B} / m$ constant. To obtain the isothermal model, one can add a cooling term $-\rho c_{V}\left(T-T_{0}\right) / t_{\text {cool }}$ to the right side of Eq. (121), with cooling time $t_{\text {cool }}$ taken to be much shorter than any fluid evolution times. In this limit for this simple cooling model, all of the heating by pressure work and viscous dissipation will be plausibly balanced by the strong cooling, so Eq. (121) reduces, at leading order, to

$$
\rho c_{V}\left(T-T_{0}\right) / t_{\mathrm{cool}}=-p \Theta+Q_{\mathrm{visc}},
$$

with temperature fluctuations of order $O\left(t_{\text {cool }}\right)$. In this limit, the viscous isothermal model provides a description consistent with energy conservation and the second law of thermodynamics, where internal energy and entropy generated by viscosity are immediately transferred to the radiation field (on time scale $t_{\text {cool }}$ ).
To verify energy conservation, recall that internal energy per volume of an isothermal ideal gas with specific heat ratio $\gamma=c_{P} / c_{V}$ is given

$$
u=\alpha n k_{B} T_{0}=c_{V} \rho T_{0}, \quad \alpha=1 /(\gamma-1)
$$

rather than by the integral (117). Since the specific heat per volume $c_{V}$ and the temperature $T_{0}$ are both considered constant, the internal energy $u$ in the isothermal model obeys the same equation as the density $\rho$, or

$$
\partial_{t} u+\nabla \cdot(u \mathbf{v})=0 .
$$

Together with Eq. (14) for the kinetic energy, this gives, for total fluid energy $E=(1 / 2) \rho v^{2}+u$, the equation

$$
\partial_{t} E+\boldsymbol{\nabla} \cdot\left((E+p) \mathbf{v}+\mathbf{T}_{\mathrm{visc}} \cdot \mathbf{v}\right)=p \Theta-Q_{\mathrm{visc}},
$$

with $\mathbf{T}_{\text {visc }}$ the viscous stress. If one uses the limiting temperature balance (122), then this becomes

$$
\partial_{t} E+\boldsymbol{\nabla} \cdot\left((E+p) \mathbf{v}+\mathbf{T}_{\mathrm{visc}} \cdot \mathbf{v}\right)=-\rho c_{V}\left(T-T_{0}\right) / t_{\mathrm{cool}},
$$

so the energy lost by the fluid is exactly that gained by radiation for the simple cooling law in the limit $t_{0} \rightarrow 0$. Note that the integral $-\int p(\rho) d(1 / \rho)$, sometimes described as internal energy in the isothermal model, in fact represents the chemical potential per mass $\mu_{m}=\mu / m$ or, equivalently, the Gibbs free energy $g$ per mass. Indeed, the Gibbs-Duhem relation $d p=s d T+\rho d \mu_{m}$ with $d T=0$ and $d(p / \rho)=0$ immediately yields $d \mu_{m}=p d \rho / \rho^{2}$. Consistently, substituting $p=\left(k_{B} T / m\right) \rho$ gives

$$
\int p(\rho) \frac{d \rho}{\rho^{2}}=\left(k_{B} T / m\right) \log \left(\rho / \rho_{0}\right)
$$

which agrees with the chemical potential per mass of an ideal gas up to an additive constant depending only upon temperature.

To verify the entropy balance, we use the well-known formula for the entropy per particle of an ideal gas,

$$
s_{n}=k_{B} \ln \left(T_{0}^{\alpha} / c n\right)
$$

From the equation $D_{t} \ln n=-\Theta$, one then readily obtains

$$
\partial_{t} s+\nabla(s \mathbf{v})=n k_{B} \Theta .
$$

Furthermore, together with the ideal-gas law $p=n k_{B} T$ and the limiting temperature relation (122) for $t_{0} \rightarrow 0$, the entropy balance becomes

$$
\partial_{t} s+\nabla(s \mathbf{v})=\frac{1}{T_{0}}\left(Q_{\text {visc }}-\rho c_{V}\left(T-T_{0}\right) / t_{\text {cool }}\right) .
$$


In the statistical steady state for this problem, therefore, the mean entropy production by viscous dissipation correctly balances with the entropy carried off by the radiation field. Our prediction of an entropy cascade does not apply to such gases that are strongly cooled at all scales (although our analysis of kinetic-energy cascade still carries over to this extreme isothermal limit). For more realistic cooling models, however, it is far less clear that an isothermal description will emerge, no matter how strong the rate of cooling. Even a quite simple Stefan-Boltzmann radiation law with $Q_{\text {cool }}=a T^{4}$ involves cooling that is nonuniform in scale and concentrated mostly at large scales, when the temperature spectrum is peaked at low wave numbers. The cooling mechanisms in a realistic astrophysical situation like the ISM are even more complex.

\section{B. Point-splitting regularization}

As discussed in the Introduction, Onsager derived his result on dissipative anomalies for incompressible Euler equations in his original work using a point-splitting regularization [20,31], closely related to the methods used by Kolmogorov [17] to derive his statistical 4/5th law for incompressible turbulence. Onsager's argument was later made completely rigorous by Duchon-Robert [42], and a formal analogy to point-splitting derivations of anomalies in quantum gauge theories was pointed out by Polyakov $[33,34]$. It is therefore natural to consider whether the point-splitting approach can be applied as well to compressible fluids. Two different groups of researchers have already attempted to obtain statistical relations analogous to the 4/5th law for compressible turbulence by distinct variations of point-splitting methods: Galtier and Banerjee [54,55] and Falkovich-Fouxon-Oz [24]. We briefly discuss the works of both of these groups in the light of our own results.

Galtier-Banerjee relations.-These authors have derived relations analogous to $4 / 5$ th laws for compressible turbulence within viscous isothermal [54] and polytropic [55] fluid models by a point splitting of the total fluid energy (kinetic + internal). Here, we present several criticisms of their analysis.

First, there are difficulties in the physical interpretation. If the integral $\int p(\rho) d \rho / \rho^{2}$ is interpreted to be internal energy per mass and $E=(1 / 2) \rho v^{2}+\rho e$ the total fluid energy, as stated in Refs. [54,55], then their results are inconsistent with conservation of energy for an isolated fluid. An alternative interpretation that the relation of Refs. [54,55] describes the flux of a barotropic energy appears to us to be also problematic for a molecular fluid, as discussed in the previous subsection. The possibility remains that the viscous isothermal or barotropic systems studied in Refs. [54,55] may be acceptable as approximate models of weakly collisional astrophysical fluids, in which the viscosity is a proxy for some other physical mechanism that transforms the cascaded kinetic energy at small scales not into internal energy of the fluid in question but into some other form (e.g., internal energy of another fluid component, electromagnetic radiation, nonthermal particle acceleration, etc.). Note that the inertial-range analysis of the present paper, and also of Refs. [23,38], applies to the viscous barotropic/isothermal models. The only change to our analysis of the inertial-range balance of $u$ in Sec. V B is that terms $Q_{\text {visc }}$ must be set to zero there (and in the isothermal model $p \Theta$ is also omitted). To justify a fluid approximation for internal energy, there must be some additional physical mechanism, not explicitly represented in Eq. (118), which regularizes its solution. For example, this could be the tiny thermal conductivity of electrons due to rare collisions with ions $[146,147]$. There may also be very weak collisional heating enhanced by kinetic mechanisms $[124,125]$. However, our analysis leads to a quite different picture than that of Galtier and Banerjee [54,55] for their own models, where we predict no cascade of internal energy. Any "cascade of total energy" is only via kinetic-energy cascade in our analysis. As pointed out in the previous section, the quantity called internal energy for the isothermal fluid in Ref. [54], $e=\int p(\rho) d \rho / \rho^{2}$, is, in fact, the Gibbs free energy $g$ per mass. One can always consider the cascade of the quantity $(1 / 2) \rho v^{2}+\rho g$, which is an inviscid invariant of the isothermal model, but it is not the physical energy.

It is also interesting to ask whether the results of the present paper on a kinetic-energy anomaly for Euler equations might be alternatively derived by the GaltierBanerjee point splitting. The answer is no because the point splitting employed by Galtier-Banerjee is not a proper regularization of the kinetic-energy equation and does not remove divergences in the infinite Reynolds-number limit. To see this, we note that the key identity in Refs. [54,55] for the point-split kinetic energy evolved under isentropic Euler dynamics is

$$
\begin{aligned}
& \partial_{t}\left(\mathbf{j} \cdot \mathbf{v}^{\prime}+\mathbf{j}^{\prime} \cdot \mathbf{v}\right) \\
& \quad \equiv \quad \nabla_{\mathbf{r}} \cdot[(\delta \mathbf{j} \cdot \delta \mathbf{v}) \delta \mathbf{v}]+h_{m}^{\prime}(\boldsymbol{\nabla} \cdot \mathbf{j})+h_{m}\left(\boldsymbol{\nabla} \cdot \mathbf{j}^{\prime}\right) \\
& \quad+\left(\mathbf{j} \cdot \mathbf{v}^{\prime}-\mathbf{j} \cdot \mathbf{v}+p\right) \boldsymbol{\nabla} \cdot \mathbf{v}^{\prime}+\left(\mathbf{j}^{\prime} \cdot \mathbf{v}-\mathbf{j}^{\prime} \cdot \mathbf{v}^{\prime}+p^{\prime}\right) \boldsymbol{\nabla} \cdot \mathbf{v}
\end{aligned}
$$

Here, quantities marked with a prime " " " are evaluated at a space point $\mathbf{x}+\mathbf{r}$, while unmarked quantities are evaluated at point $\mathbf{x}$, and $\delta f$ is the difference $f(\mathbf{x}+\mathbf{r})-f(\mathbf{x})$. The notation " $\equiv$ " indicates equality up to overall space-gradient terms $\boldsymbol{\nabla}_{\mathbf{x}} \cdot(\ldots)$, which represent space transport of kinetic energy. Finally, $h_{m}=e+p / \rho$ is the enthalpy per mass, which satisfies $d h_{m}=d p / \rho$ for isentropic flow. In the original work of Onsager [20,31] and Duchon-Robert [42], a coarse-graining operation was applied to the separation vector $\mathbf{r}$ to obtain a fully regulated expression. However, if the same approach is applied to the above identity, one gets terms that are ill defined in the infinite Reynolds-number 
limit. For example, the last term on the right gives the contribution $(\overline{\mathbf{j}} \cdot \mathbf{v}-\overline{\mathbf{j} \cdot \mathbf{v}}+\bar{p}) \boldsymbol{\nabla} \cdot \mathbf{v}$, which involves a nonsmooth function $\mathbf{v}$ multiplied by a distribution $\boldsymbol{\nabla} \cdot \mathbf{v}$. Just as in local quantum field theory where UV divergences likewise arise from the attempt to multiply distributions at the same space-time point (e.g., see Ref. [148], Sec. 23.1), such products of "bare fields" are a priori ill defined at infinite Reynolds number. Instead, the coarse-graining approach of Ref. [23] and the present paper yields fully regularized expressions, as in our Eq. (43). Notice that the terms that cause trouble for point splitting as a regularizer are absent in the incompressible case because $\boldsymbol{\nabla} \cdot \mathbf{v}=0$.

This is not just an abstract technical problem, but, in fact, a correct regularization leads to results that are quite different from those of Refs. [54,55]. We can illustrate this statement for the balance of the inviscid invariant $I=(1 / 2) \rho|\mathbf{v}|^{2}+\rho w(\rho)$ of the general barotropic model, with $w=-\int p d(1 / \rho)$. [Of course, we have argued above that the viscous barotropic model is physically inconsistent as a model of an isolated molecular fluid, but one can nonetheless consider the mathematical problem of the high Reynolds-number limit of this model. For the case $p(\rho) \propto \rho$, the analysis yields exact 4/5th laws for the total Gibbs free energy of an isothermal ideal gas.] We can use the same Favre coarse graining that we employed for compressible Navier-Stokes. A regularization of the invariant $I$ can then be defined by

$$
\underline{I}=(1 / 2) \bar{\rho}|\tilde{\mathbf{v}}|^{2}+\bar{\rho} w(\bar{\rho}) .
$$

It is now straightforward, using the viscous barotropic fluid equations, to derive a balance equation for $\underline{I}$, which, in the inviscid limit $\eta, \zeta \rightarrow 0$ (for simplicity), becomes

$$
\begin{aligned}
\partial_{t} \underline{I} & +\boldsymbol{\nabla} \cdot[(\underline{I}+\bar{p}) \tilde{\mathbf{v}}+\bar{\rho} \tilde{\tau}(\mathbf{v}, \mathbf{v}) \cdot \tilde{\mathbf{v}}] \\
& =\Delta p(\boldsymbol{\nabla} \cdot \tilde{\mathbf{v}})+\bar{\rho} \boldsymbol{\nabla} \tilde{\mathbf{v}}: \tilde{\tau}(\mathbf{v}, \mathbf{v}):=-\Pi_{\ell}^{\text {flux }},
\end{aligned}
$$

with $\Delta p=\bar{p}-\underline{p}$. In contrast to the point-split equations of Refs. [54,55], all of the terms in Eq. (133) are perfectly well defined in the limit $\eta, \zeta \rightarrow 0$ with $\ell$ fixed. The terms on the right-hand side of Eq. (133) define the flux $\Pi_{\ell}^{\text {flux }}$ of the invariant $I$ and describe its inertial-range turbulent cascade. Using the cumulant-expansion methods of our paper, this flux can be written in terms of increments, thus providing the exact analogue of a $4 / 5$ th law for this problem. In particular, the estimates (49), (50), and (79) in our paper yield

$$
\begin{aligned}
& (\Delta p)(\boldsymbol{\nabla} \cdot \tilde{\mathbf{v}})=O\left(\frac{(\delta \rho)^{2}(\delta v)}{\ell}\right), \\
& \bar{\rho} \boldsymbol{\nabla} \tilde{\mathbf{v}}: \tilde{\tau}(\mathbf{v}, \mathbf{v})=O\left(\frac{(\delta v)^{3}}{\ell}\right)
\end{aligned}
$$

We emphasize that the point-splitting approach employed in Refs. [54,55] does not, and cannot, yield the analogue of Eqs. (133) and (134). Instead, the results described above are a slight simplification of those in Ref. [149], which employs the same coarse-graining regularization as in our work.

Just to be clear, we are not claiming that there is a mathematical mistake of a trivial sort in the analyses of Galtier and Banerjee [54,55]. All of their calculations are meaningful and correct at finite Reynolds numbers. In fact, their mathematical relations have been checked to be true in numerical simulations of supersonic "isothermal" turbulence [140]. What we are claiming is that there are unphysical assumptions underlying the mathematical models employed by Galtier and Banerjee [54,55] and erroneous physical interpretations of the mathematical results. Their failure to regularize UV divergences associated with dissipative anomalies prevents them from drawing any conclusions on the infinite Re limit.

Falkovich-Fouxon-Oz relation.-In the paper of Falkovich et al. [24], another generalization of the 4/5th law to compressible turbulence has been obtained for a barotropic fluid. This approach has also been applied to relativistic fluid turbulence by Fouxon and $\mathrm{Oz}$ [25], as we will discuss in a following paper [56]. Consideration of a point-split quantity $\mathbf{j} \cdot \mathbf{j}^{\prime}$ allowed Ref. [24] to derive an exact relation for homogeneous, isotropic statistics, which reduces to the standard 4/5th law in the incompressible limit. The quantity that is cascaded to small scales in their picture is the input of $(1 / 2)|\mathbf{j}|^{2}$ by external forcing. The exact equation obeyed by this field for a smooth solution of compressible Euler equations (without the need for any barotropic assumption) is

$$
\begin{gathered}
\partial_{t}\left(\frac{1}{2}|\mathbf{j}|^{2}\right)+\boldsymbol{\nabla} \cdot\left(\frac{1}{2}|\mathbf{j}|^{2} \mathbf{v}+p \mathbf{j}\right) \\
\quad=-\frac{1}{2}|\mathbf{j}|^{2}(\boldsymbol{\nabla} \cdot \mathbf{v})+p(\boldsymbol{\nabla} \cdot \mathbf{j})
\end{gathered}
$$

As a matter of fact, it is not hard to show that this balance equation may indeed be anomalous in a high Reynolds-number compressible turbulence or to use a point-splitting regularization to derive the anomaly. With the same notations as in Eq. (131), one easily finds

$$
\begin{aligned}
\partial_{t}\left(\mathbf{j} \cdot \mathbf{j}^{\prime}\right) \equiv & \frac{1}{2} \boldsymbol{\nabla}_{\mathbf{r}} \cdot\left[|\delta \mathbf{j}|^{2} \delta \mathbf{v}\right]-\frac{1}{2}|\mathbf{j}|^{2}\left(\boldsymbol{\nabla} \cdot \mathbf{v}^{\prime}\right)-\frac{1}{2}\left|\mathbf{j}^{\prime}\right|^{2}(\boldsymbol{\nabla} \cdot \mathbf{v}) \\
& +p\left(\boldsymbol{\nabla} \cdot \mathbf{j}^{\prime}\right)+p^{\prime}(\boldsymbol{\nabla} \cdot \mathbf{j}) .
\end{aligned}
$$

Unlike the previous case, all terms are fully regularized after coarse graining in the separation vector $\mathbf{r}$, and one obtains an anomaly term $-A$ appearing on the right side of Eq. (135) for infinite Reynolds number, with 


$$
A=\mathcal{D}-\lim _{\ell \rightarrow 0} \frac{1}{4 \ell} \int d^{d} r(\nabla G)_{\ell}(\mathbf{r}) \cdot \delta \mathbf{v}(\mathbf{r})|\delta \mathbf{j}(\mathbf{r})|^{2} .
$$

It is also straightforward to derive the anomalous balance equation for $(1 / 2)|\mathbf{j}|^{2}$ by using the coarse-graining approach of the present paper, but we leave this as an exercise for the reader. Note that, in this balance equation, one faces the same issue of defining products like $\frac{1}{2}|\mathbf{j}|^{2} \circ \Theta$ and $\frac{1}{2}|\mathbf{j}|^{2} * \Theta$, similar to pressure work in the energy balances in Sec. $\mathrm{V}$ of the present paper.

As with the previous point-splitting approach, we conclude that the result of Falkovich et al. [24] is mathematically correct, and, moreover, the derivation is valid in the infinite Reynolds-number limit. The statistical relation of Ref. [24] has also been verified in a numerical simulation of isothermal compressible turbulence [150] (although there are some subtle issues in the statistical evaluation of the external input). However, we disagree with the conclusion that the result of Ref. [24] "...indicates that the interpretation of the Kolmogorov relation for the incompressible turbulence in terms of the energy cascade may be misleading" (Fouxon and Oz [25]). Such a conclusion could be justified if that relation were the only possible generalization of the 4/5th law to compressible turbulence. The analysis of Refs. $[23,38]$ and ours in Sec. VA of the present paper instead fully support the existence of a kinetic-energy cascade for compressible turbulence and yield the analogue of 4/5th laws for the kinetic-energy flux. A further issue with the result of Falkovich et al. [24] is that we see no compelling interest in the quantity $(1 / 2)|\mathbf{j}|^{2}$ for compressible fluids. It is neither a conserved quantity nor any component of a conserved quantity, and it has no obvious dynamically important role in compressible turbulence. Just as in quantum field theory, it is not hard to find infinitely many anomalous balance relations in the ideal limit of turbulence, but most of them are not physically relevant and have no significant consequences. In our opinion, the deep importance of the 4/5th law for incompressible turbulence arises from its connection to the dissipative anomaly for kinetic energy and its implication that fluid singularities of the type $\zeta_{q}^{v} \leq q / 3$ are required for such an anomaly. Our analysis shows that such a connection fully extends to compressible fluid turbulence.

As our final comments in this section, we would like to emphasize the general limitations of point splitting in turbulence theory. It is not ruled out by the analysis in this paper that a clever point splitting may someday be found for compressible turbulence, which will yield the anomalous kinetic-energy balance (54). However, it is hard to imagine that a point-splitting regularization will ever be found to yield the anomalous entropy balance (84) in this paper. The coarse-graining approach that we employ is a more powerful and general method than point splitting. In addition to the Eulerian balances discussed here, coarse graining can also be employed to obtain Lagrangian conservation-law anomalies, such as for fluid circulation in hydrodynamic turbulence [86] and magnetic flux conservation in MHD turbulence $[89,151]$.

\section{Decomposition into linear wave modes}

Another common theoretical approach to compressible turbulence, which goes back to the work of Kovásznay [52] and Chu and Kovásznay [53], is to expand compressible Navier-Stokes solutions into linear wave modes, based on an assumption of small perturbations around a homogeneous state and weak nonlinearity. This expansion identifies three ideal linear wave modes [152]: the "sound mode" of frequency $c_{s} k$ for sound speed $c_{s}$ and wave number $k$, and two zero-frequency modes-the "vorticity mode" and the "entropy mode." An obvious question, which we address here, is how the entropy mode of Kovásznay is related to our concept of an entropy cascade.

To briefly review the approach of Kovásznay [52] and Chu and Kovásznay [53], we recall that it assumes an ideal-gas equation of state, with pressure and entropy per particle given by

$$
p=n k_{B} T, \quad s_{n}=k_{B} \log \left(T^{\alpha} / C n\right)
$$

as functions of $n$ and $T$, with $\alpha=1 /(\gamma-1)$. Linearization around a homogeneous state satisfying $p_{0}=n_{0} k_{B} T_{0}$ yields, for the fluctuations, the linear relations

$$
\frac{p^{\prime}}{p_{0}}=\frac{n^{\prime}}{n_{0}}+\frac{T^{\prime}}{T_{0}}, \quad \frac{s_{n}^{\prime}}{k_{B}}=-\frac{n^{\prime}}{n_{0}}+\alpha \frac{T^{\prime}}{T_{0}} .
$$

Here, we use the prime " " to denote a putatively small fluctuation value. For ideal flow, the sound mode has $s_{n}^{\prime}=$ 0 and the entropy mode has $p^{\prime}=0$. (For nonideal flow, Kovásznay finds instead a small entropy $s_{n}^{\prime}$ associated with the sound mode, which is proportional to the molecular transport coefficients or dimensionless "Kundsen number" $\epsilon$ and which is neglected at zeroth order in $\epsilon$.) The zerothorder dynamics of the fluctuations for ideal flow are found to be given by the linear equations

$\partial_{t} \boldsymbol{\omega}^{\prime}=\mathbf{0}, \quad \partial_{t} s_{n}^{\prime}=0, \quad \partial_{t}^{2} p^{\prime}-c_{s}^{2} \nabla^{2} p^{\prime}=0$,

with $\boldsymbol{\omega}^{\prime}=\boldsymbol{\nabla} \times \mathbf{v}^{\prime}$ the vorticity fluctuation. See Ref. [53], Eq. (6.5). Nonlinearity is recovered in the Kovásznay approach by expansion to second order in the nonlinearity, which yields mode-mode coupling terms, such as vortex self-stretching (a vorticity-vorticity mode coupling). See Table 1 of Ref. [53] for a complete tabulation of all secondorder interactions. The only such couplings that contribute to entropy dynamics are entropy-vorticity and entropysound couplings of the form $-\mathbf{v}^{\prime} \cdot \boldsymbol{\nabla} s_{n}^{\prime}$, which describe advection of entropy by velocity fluctuations $\mathbf{v}^{\prime}$ due to vorticity and sound modes. Thus, to quadratic order in nonlinearity, the entropy per particle $s_{n}^{\prime}$ appears as a passive 
scalar and entropy per volume $s^{\prime}=n s_{n}^{\prime}$ as a passive density.

Independent of our work, there are a number of serious problems with the Kovásznay modal expansion when considered as an a priori theoretical approach. First and foremost, there is no small parameter on which to base such an expansion. Instead, fluctuations of thermodynamic variables in compressible flow can be very large relative to mean or r.m.s. values, as seen, for example, in Ref. [145], Fig. 4 for $M a=0.1-0.6$. For very small Mach numbers, density and pressure fluctuations may become small; however, even in that limit, Eqs. (20) and (21) of the velocity and temperature fields remain strongly nonlinear, and anomalous entropy production occurs by turbulent cascade [153]. This essential strong-coupling nature is, of course, the most well-known theoretical difficulty with the analysis of turbulent flow. A closely related problem is that solutions of the compressible Navier-Stokes equation cannot be consistently expanded into linear wave modes because there is no superposition principle for such nonlinear dynamics. Even for an ideal gas, the thermodynamic relations (138) impose nonlinear constraints between $p, n$, $T$ or $s_{n}, n, T$, which will not be satisfied for superpositions of wave modes except in the crude linear approximation (139). For second-order moments of $p^{\prime} / p_{0}$ and modest Mach numbers (0.1-0.6), the predictions of the linear approximation (139) are adequate to about the 1\% level (see data in Table 1 and Figs. 2 and 3 in Ref. [145]), but the error grows with increasing Mach number and also for higher moments or larger fluctuations. It is worth noting that Kovásznay himself was not attempting to develop a general theoretical approach for analysis of compressible fluid turbulence in his original works, but his goal was instead a more modest one of constructing a decomposition to assist in the interpretation of experimental measurements. Some later researchers have taken this type of modal decomposition much more literally than it was first intended.

Our analysis in this paper has shown that the entropy in high Reynolds-number compressible turbulence is not at all a passive scalar. Entropy is, of course, a nonlinear function of basic thermodynamic variables, e.g., $s(u, n)$ taken as a function of internal energy density $u$ and particle density $n$. Its dynamics is completely determined by the dynamics of $u$ and $n$, and it is, in that sense, "passive." However, the turbulent dynamics of entropy in the ideal limit of vanishing molecular transport is not that of a passive scalar but instead remains fundamentally constrained by the second law of thermodynamics. Comparing the weakly nonlinear expansion result

$$
\partial_{t} s^{\prime}+\nabla \cdot\left(s^{\prime} \mathbf{v}^{\prime}\right)=0
$$

with our own Eq. (84), we see that, beyond passive advection, the inertial-range dynamics of entropy involves both anomalous input $I_{\text {mech }}$ of negentropy from pressure work and nonlinear entropy cascade $\Sigma_{\text {flux }}$ (as well as entropy production by viscous heating). If one tries to interpret the mechanical input $I_{\text {mech }}$ of entropy crudely within the Kovásznay framework, it would have to be considered a turbulent "sound-sound" coupling that produces negentropy. This is completely missed by the Kovásznay weakly nonlinear expansion, which cannot detect such "anomalous" terms. Finally, the identification of $s_{n}^{\prime}$ as a passive scalar would imply that there is a forward cascade of $\left|s_{n}^{\prime}\right|^{2}$, but our analysis instead predicts an inverse cascade of the entropy $s$ as a nonlinear function of $u$ and $n$. Our predictions for entropy are thus fundamentally different from those obtained by treating the linear entropy mode as a passive scalar.

\section{EMPIRICAL CONSEQUENCES AND EVIDENCE}

Our analysis yields a great many predictions testable by laboratory experiments and numerical simulations, the two most novel being the pressure-dilatation defect $\tau_{\ell}(p, \Theta)$ contribution to anomalous kinetic-energy dissipation and the anomalous production of negentropy by pressure work $I_{\ell}^{\text {flux }}$ and nonlinear negentropy cascade $\Sigma_{\ell}^{\text {flux* }}$. These quantities are all straightforward to calculate in simulations of compressible turbulence, where inertial-range contributions such as $\bar{\tau}_{\ell}(p, \Theta)$ or negentropy flux $\Sigma_{\ell}^{\text {flux* }}$ can be obtained by numerical implementation of the spatial filtering. Laboratory experiments can also measure such quantities using techniques such as holographic particle-imaging velocimetry [154]. Our analysis also yields testable predictions on scaling exponents through the inequalities (94)-(96). Here, we may note, in particular, the predictions for "roughness" of the internal energy density and mass density fields in order for an entropy cascade to exist, with structure-function exponents $\zeta_{q}^{u}$ and $\zeta_{q}^{\rho}$ essentially required to be less than or equal to K41 values for $q \geq 3$. These predictions can not only be checked in detail in future studies, but also many past works, in retrospect, provide supporting evidence. We next discuss some of this prior work.

First, the previous numerical studies of the pressure work $[104,105]$ provide evidence for a pressure-work defect $\tau(p, \Theta)$, although this was not clearly understood at the time. The main object of those studies was the saturation of $\left\langle\bar{p}_{\ell} \bar{\Theta}_{\ell}\right\rangle$ for $\ell$ decreasing through the inertial range. This was demonstrated through the study of the pressuredilatation cospectrum

$$
P D(k)=-\sum_{\mathbf{k}^{\prime}:\left|\mathbf{k}^{\prime}\right|-k \mid<0.5} \hat{p}\left(\mathbf{k}^{\prime}\right) \hat{\Theta}\left(-\mathbf{k}^{\prime}\right)
$$

and of the statistics of the pressure-dilatation residual $p \Theta-\bar{p} \bar{\Theta}$. In both studies [104,105], it was found 
that the cospectrum exhibited a power-law behavior $P D(k) \sim C k^{-\beta}$ in the inertial range, crucially with $\beta>1$, so the integral over the range $k \in[0, \infty)$ would converge. However, in the finite Reynolds-number simulations, the power law with exponent $\beta$ persists only over a finite range, and in the dissipation range, the cospectrum was found to lie above the inertial-range power law. See Ref. [104], Fig. 2, and Ref. [105], Fig. 23. This is the signature to be expected from a positive mean defect $\langle\tau(p, \Theta)\rangle>0$. Even more relevant are the previous numerical results for the pressure-dilatation residual since it is directly related to the mean defect by

$$
\langle\bar{\tau}(p, \Theta)\rangle=\langle p \Theta-\bar{p} \bar{\Theta}\rangle .
$$

The two previous studies both found that the residual for $\ell$ near the bottom of the inertial range took on very large positive and negative values associated with shocks (smallscale shocklets or large-scale shocks, depending upon the compressibility of the forcing). See Ref. [104], Fig. 4, and Ref. [105], Figs. 26 and 27. The large values nearly cancel in a global space average, leaving only a small positive average $\langle\tau(p, \Theta)\rangle$, about 20 times smaller than the asymptotic value $\langle p \circ \Theta\rangle$. Aluie et al. [104] considered this 5\% contribution to be "negligible." However, both the simulations $[104,105]$ were for subsonic and transonic turbulence. If $\langle\tau(p, \Theta)\rangle$ arises mainly from shock heating, then it is reasonable to expect that this average will make an increasingly large contribution to the kinetic-energy dissipation anomaly for increasing Mach numbers.

There is also evidence from prior studies for a negentropy cascade. Motivated by incompressible fluid turbulence where the temperature is a passive scalar, Ni et al. [77] and Ni and Chen [105] (see Sec. VI of both papers) have numerically studied "temperature cascade" in subsonic and transonic compressible turbulence of an ideal gas. Using the same coarse-graining approach as the present paper, those authors attempted to derive a balance equation for the quantity $G=(1 / 2) \bar{\rho} \tilde{T}^{2}$. Their result [Ref. [105], Eqs. (6.3)-(6.8)] contains several errors [155], but the expression for the subscale flux of $G$ that they obtained is the same as that for the correct equation derived from Eq. (121) and given here:

$$
\begin{array}{r}
\partial_{t}\left(\frac{1}{2} \bar{\rho} \tilde{T}^{2}\right)+\boldsymbol{\nabla} \cdot\left(\frac{1}{2} \bar{\rho} \tilde{T}^{2} \tilde{\mathbf{v}}+\bar{\rho} \tilde{\tau}(T, \mathbf{v}) \tilde{T}-(\overline{\kappa \nabla} T) \tilde{T} / c_{V}\right) \\
\quad=-\Pi_{\ell}^{G}+\left(-\overline{p \Theta}+\bar{Q}_{\text {visc }}\right) \tilde{T} / c_{V}-\nabla \cdot(\overline{\kappa \nabla} T) \cdot \boldsymbol{\nabla} \tilde{T} / c_{V},
\end{array}
$$

with

$$
\Pi_{\ell}^{G}=-\bar{\rho} \mathbf{\nabla} \tilde{T} \cdot \tilde{\tau}(T, \mathbf{v})
$$

the subscale flux of $G$. Studies [77,105] have verified numerically that this quantity has a positive average
$\left\langle\Pi_{\ell}^{G}\right\rangle>0$ over a range of $\ell$, indicating a forward cascade of the quantity $G$ to small scales. Given that the quantity $G=\frac{1}{2} \bar{\rho} \tilde{T}^{2}$ was first introduced by Obukhov [80] for incompressible fluid turbulence as an approximation to the "negentropy" or "information" introduced by an ordered temperature field, this is almost direct evidence for a forward negentropy cascade.

For compressible turbulence, there is little reason to consider the approximation $G$ rather than the correct large-scale entropy $s(\bar{u}, \bar{\rho})$, and our balance equations (74) for $\underline{s}$ and (98) for $\underline{s}^{*}$ are more theoretically tractable than Eq. (144) for $G$ because entropy is a conserved quantity for smooth solutions of compressible Euler equations whereas $G$ is not. However, the observation of Refs. [77,105] that $\left\langle\Pi_{\ell}^{G}\right\rangle>0$ strongly suggests that $\left\langle\Sigma_{\ell}^{\text {flux }}\right\rangle>0$ will hold over a similar range of $\ell$ and makes it vital to subject the latter prediction and the balance relations (107), (108), (111), and (112) to detailed empirical tests. Here, we note that the spectra of density, temperature, and pressure (or, equivalently, for an ideal gas, internal energy) in the simulations of Refs. [77,145] are consistent with the roughness expected for negentropy cascade. In particular, for transonic Mach numbers $(M a \approx 0.6)$, all three thermodynamic variables have Fourier spectra close to $k^{-5 / 3}$, scaling with the K41 exponent [156].

The negentropy cascade proposed here, if correct, must occur for compressible turbulent flows in nature, with one of the most significant examples being turbulence in the ISM. The electron density of the ISM exhibits a spectrum close to the Kolmogorov $k^{-5 / 3}$ over a 13 -decade range, as inferred from electron scintillation measurements over $10^{5}-10^{10} \mathrm{~km}$ scales and from other observations over $10^{2}-10^{15} \mathrm{~km}$ [157-160]. The spectacular extent of this scaling range has led to the density spectrum being dubbed the "Big Power Law in the Sky." Because the ion mean free path in the ISM is about $10^{7} \mathrm{~km}$, a fluid approximation is expected to be valid over the majority of this range. Magnetic fields also play a significant role in the dynamics of the ISM, so the dynamics of the ISM at length scales above about $10^{7} \mathrm{~km}$ is expected to be that of a compressible magnetohydrodynamic (MHD) fluid with a Mach number of order unity. Our work suggests an identification of the Big Power Law in the Sky as resulting from a nonlinear inverse cascade of entropy (or forward negentropy cascade). Note that all of our results in this paper extend straightforwardly to compressible MHD (see Landau and Lifshitz [161], Chap. VIII, Secs. 65-66). The only difference is that now there is a cascade of total mechanical energy (kinetic + magnetic), and the corresponding energy dissipation anomaly now contains a contribution from resistive heating,

$$
\bar{Q}=\lim _{\eta, \zeta, \kappa, \gamma \rightarrow 0} \overline{2 \eta|\mathbf{S}|^{2}+\zeta \Theta^{2}+\gamma J^{2} / 4 \pi}
$$


where $\gamma=c^{2} / 4 \pi \sigma$ is the magnetic diffusivity and $\mathbf{J}=\boldsymbol{\nabla} \times \mathbf{B}$. In particular, our balance equation (74) for $\underline{s}$ remains valid for compressible MHD with the above change to $Q_{\text {diss}}$, and the balance equation (98) for $\underline{s}^{*}$

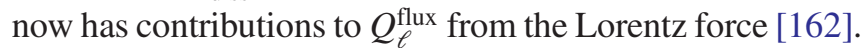
Thus, by our arguments, there is a forward negentropy cascade in compressible MHD turbulence. We theorize that this nonlinear negentropy cascade is the origin of the plentiful density fluctuations in the large scales of the ISM where compressible MHD is valid.

Most current theories of the electron density spectrum of the ISM, by contrast, have been developed within a Kovásznay-type linear wave-mode picture for compressible MHD, where the basic waves are now the "shear Alfvén mode," the "slow magnetosonic mode," the "fast magnetosonic mode," and the "entropy mode" (e.g., see Ref. [163], Chap. 5). In particular, one popular theory of the power-law spectrum is that it results from a forward cascade of the entropy mode as a passive scalar $[164,165]$. However, the large scales of the ISM are believed to be nearly isothermal above a cooling scale $L_{\text {cool }} \sim 10^{12} \mathrm{~km}$ because of efficient radiative cooling (e.g., by electron impact excitation of metal line transitions). Referring to Eq. (139), one sees that there can then be no entropy mode with $p^{\prime} / p_{0}=0$ because $T^{\prime} / T_{0}=0$ and so cannot cancel the density fluctuation $n^{\prime} / n_{0}$ (or, more accurately, the entropy mode is extremely damped because $\left.T^{\prime} / T_{0} \ll 1\right)$. In that case, the only remaining mode to carry density fluctuations is the isothermal sound mode (slow magnetosonic) with $p^{\prime} / p_{0}=n^{\prime} / n_{0}=$ $-s_{n}^{\prime} / k_{B}$ (which, in contrast to the adiabatic sound mode, carries entropy fluctuations due to density changes) [166]. This linear analysis of the fluctuations leads to the so-called cooling catastrophe, which is concisely summarized in this quote:

\begin{abstract}
"However, the entropy mode is rapidly damped in isothermal turbulence. As a consequence, small-scale density fluctuations may be significantly suppressed. There are two possible solutions to this "cooling catastrophe": either (1) the outer scale is extremely small, small enough that the turbulence at the outer scale is nearly adiabatic; or (2) there are significant density fluctuations associated with the slow mode. However, in the latter case, the mean magnetic field must be amplified almost to equipartition with the gas pressure, so that $\beta \sim 1$. Either of these two solutions would place stringent constraints on the nature of the turbulence that is responsible for observed density fluctuations."-Lithwick and Goldreich [165].
\end{abstract}

Within a Kovásznay-type modal picture, the slow magnetosonic mode seems the most plausible source of the observed density fluctuations. However, in our nonlinear theory, there is no cooling catastrophe in the first place. Large-scale cooling adds excess negentropy (deficiency of entropy) that feeds the cascade of negentropy to small scales. See Eq. (107). This necessitates "rough" density and temperature fields with Kolmogorov-type spectra. In our view, the cooling catastrophe is an artifact of attempting to describe nonlinear compressible MHD turbulence in terms of linear wave modes. There is no sound theoretical basis for such a decomposition and, unsurprisingly, the Kovásznay modemode interactions lead to empirically wrong predictions for the problem [167].

A complete presentation of this theory of the ISM electron density spectrum will be given elsewhere, as it requires more specialized discussion of MHD turbulence and even plasma kinetics. An interesting question is how our theorized negentropy cascade proceeds to smaller length scales below the ion mean free path where a fluid approximation breaks down. As discussed earlier, the observed $k^{-5 / 3}$ density spectrum in the ISM extends many decades below the ion mean-free-path length. The key concept of plasma kinetic turbulence is the cascade of negative kinetic entropy or "free energy" (electromagnetic energy minus kinetic entropy) to small scales of length and velocity in the one-particle phase space $[124,125]$. The natural conjecture is that the negentropy cascade of compressible MHD turbulence merges with the kinetic cascade at scales below the mean free path, but details remain to be understood.

\section{DISCUSSION}

The theory developed in this paper is based upon the hypothesis that compressible fluid turbulence should exhibit dissipative anomalies of energy and entropy, similar to those observed for incompressible fluids. From this hypothesis alone, we have argued that the high Reynoldsand Péclet-number limits should be governed by distributional or coarse-grained solutions of the compressible Euler equations. The argument closely follows that of Onsager [20,31] for incompressible fluids, which we have explained as a nonperturbative application of the principle of renormalization-group invariance. The theory makes many predictions that are testable by experiment and simulationsin particular, (1) anomalous dissipation of kinetic energy by local energy cascade and by a pressure-work defect; (2) anomalous input of negentropy into the inertial range of compressible fluid turbulence by pressure work, in addition to any external input by large-scale cooling mechanisms; (3) negentropy cascade to small scales through a flux of intrinsic inertial-range entropy; and (4) fluid singularities required to sustain cascades of energy and entropy so that at least one of Eqs. (94)-(96) must hold.

It should be stressed that even for incompressible fluids, many difficult mathematical questions remain open concerning Onsager's theory of "ideal turbulence" described by dissipative Euler solutions, and its main support arises from successful agreement with a broad array of numerical simulations and laboratory experiments. The convex integration theory $[43,44]$ has revealed that the Cauchy 
problem for incompressible Euler equations has nonunique dissipative solutions with fixed initial data, suggesting that the infinite-Reynolds turbulent solutions are essentially unpredictable. So far, no dissipative Euler solutions of the type conjectured by Onsager have been mathematically derived from incompressible Navier-Stokes solutions by the physical limit of vanishing viscosity or infinite Reynolds number. Work on toy "shell models" suggests that this limit will be very subtle and that the limiting Euler solutions will be nonunique and stochastic [67-69]. Further surprises and new insights are doubtlessly in store. However, Onsager's theory for incompressible fluid turbulence has much more empirical support than many other highly regarded physical theories, e.g., Einstein's theory of general relativity.

For the compressible theory that we have developed here, further work is also clearly required on a few key issues. One of these is the Mach-number dependence of the various physical quantities in our theory. All of our derivations are formally independent of Mach number, but there is an implicit Mach-number dependence through the assumption that mass density $\rho$ remains a bounded function in the ideal limit. Instead, there is empirical evidence from numerical simulations that, for a sufficiently high Mach number, the density is not even square integrable, and its ideal limit may exist only as a singular measure [113]. At small Mach numbers, our theoretical analysis remains valid without further assumption and recovers, as a special case, the negentropy cascade predicted long ago by Obukhov [80] for incompressible fluids with strong thermal forcing. However, the various quantities that appear in our analysis will have an explicit $M a$ dependence that is not predicted by our present considerations. For example, the anomalous negentropy input by pressure work $I_{\ell}^{\text {mech }}$ in Eq. (76) must tend to zero, but the rate of approach to the incompressible limit is unknown. As argued convincingly in Ref. [83], the small $M a$ limit is quite rich, with different limiting regimes possible, and the Mach-number dependences are presumably distinct and must be calculated separately for each. This is an urgent matter for evaluating the theory since much empirical data exist for subsonic and transonic flows.

A second very important open issue has to do with the extension of our theory to kinetic regimes. Our theorem on turbulent entropy dissipation anomalies and entropy cascade applies to any distributional solution of compressible Euler equations, including those resulting from a kinetic equation. However, it is very unclear how our fluid negentropy cascade will merge into a kinetic description at scales much smaller than the mean free path of the fluid. This is a particularly important issue for plasma kinetics in astrophysics $[124,125]$ because the large mean free paths frequently encountered in astrophysical plasmas imply that long ranges of scales are described by Vlasov-Landau kinetic theory rather than a fluid description.
One strength of our theory is that it extends readily to relativistic fluid turbulence. This is the subject of our following paper [56].

\section{ACKNOWLEDGMENTS}

We thank Ethan Vishniac and Itzhak Fouxon for discussions about compressible turbulence and the ISM, and Hussein Aluie for sharing his unpublished work with us.

\section{APPENDIX A: ANALYTICAL SHOCK SOLUTION}

\section{Model and shock solution}

We consider a family of shock solutions derived by Becker [114] and Johnson [115,169] for the 1D compressible Navier-Stokes system, obtained by reduction of the 3D equations to a single space dimension, with $x$ the distance perpendicular to the planar shock and with $v=v_{x}$ the corresponding velocity component:

$$
\begin{gathered}
\partial_{t} \rho+\partial_{x}(\rho v)=0, \\
\partial_{t}(\rho v)+\partial_{x}\left(\rho v^{2}+p-\eta \partial_{x} v\right)=0, \\
\partial_{t}\left(\frac{1}{2} \rho v^{2}+u\right)+\partial_{x}\left(\rho v\left(\frac{1}{2} v^{2}+h_{m}\right)-\eta \partial_{x} v-\kappa \partial_{x} T\right)=0 .
\end{gathered}
$$

Here, $\eta=(4 / 3) \eta_{3 \mathrm{D}}+\zeta_{3 \mathrm{D}}$ for the 3D shear viscosity $\eta_{3 \mathrm{D}}$ and bulk viscosity $\zeta_{3 \mathrm{D}}$ [170]. An ideal-gas equation of state is assumed, with

$$
p=(\gamma-1) u, \quad u=c_{V} \rho T, \quad h_{m}=c_{P} T
$$

for any adiabatic index $\gamma=c_{P} / c_{V}>1$. The solutions obtained are for the stationary equations, with all time derivatives set to zero, and they reduce in the ideal limit $(\eta, \kappa \rightarrow 0)$ to stationary shocks with discontinuous, stepfunction solution fields:

$$
f(x)=\left\{\begin{array}{ll}
f_{0} & x<0 \\
f_{1} & x>0
\end{array}=f_{0}+(\Delta f) \theta(x)\right.
$$

Here, preshock values are labeled by 0 and postshock values by $1, \Delta f=f_{1}-f_{0}$, and $\theta(x)$ is the Heaviside step function. We also denote $f_{\mathrm{av}}=\frac{1}{2}\left(f_{0}+f_{1}\right)$. The values of the fields on the two sides of the shock are related by the Rankine-Hugoniot conditions:

$\Delta(\rho v)=0, \quad \Delta\left(\frac{1}{2} v^{2}+h_{m}\right)=0, \quad \Delta\left(p+\rho v^{2}\right)=0$,

with a mass flux $j_{*}=\rho_{0} v_{0}=\rho_{1} v_{1}>0$. See Ref. [79], Sec. 84. The strength of the shock is characterized by the compression ratio $R=\rho_{1} / \rho_{0}=v_{0} / v_{1}>1$, which, for an ideal gas, is given by 


$$
R=\frac{\gamma+1}{(\gamma-1)+2 / M_{0}^{2}}
$$

in terms of the preshock Mach number $M_{0}=v_{0} / c_{s}>1$. For example, see Landau and Lifshitz [79], Sec. 89. Note that, because of the ideal-gas relation $p / \rho=(\gamma-1) h_{m} / \gamma$, the second two Rankine-Hugoniot conditions determine the preshock and postshock pressures by the formulas

$$
p_{i}=\frac{j_{*}}{2 \gamma}\left[(1+\gamma) v_{1-i}+(1-\gamma) v_{i}\right], \quad i=0,1 .
$$

As was first noted by Becker [114], the stationary 1D Navier-Stokes equations of an ideal gas admit an exact integral for $\eta=\kappa / c_{P}$ or, assuming $\zeta_{3 \mathrm{D}}=0$, for the 3D Prandtl number $\operatorname{Pr}=c_{P} \eta_{3 \mathrm{D}} / \kappa=3 / 4$. This integral takes the form of a (nonideal) Bernoulli equation, which relates velocity and enthalpy per mass:

$\frac{1}{2} v^{2}+h_{m}=\frac{1}{2} v_{0}^{2}+h_{m 0}=\frac{\gamma+1}{2(\gamma-1)} v_{0} v_{1}, \quad \operatorname{Pr}=3 / 4$.

By means of this relation and the formula

$$
\rho=j_{*} / v
$$

for the mass density, all thermodynamic variables can be related to the velocity. For example, using Eq. (A9) and the ideal-gas relation $p / \rho=(\gamma-1) h_{m} / \gamma$, we get, for the pressure field,

$$
p=\frac{j_{*}}{2 \gamma}\left[(\gamma+1) \frac{v_{0} v_{1}}{v}+(1-\gamma) v\right], \quad \operatorname{Pr}=3 / 4 .
$$

Using $h_{m}=c_{P} T$, we get, for the temperature field,

$$
T=\frac{1}{2 c_{P}}\left[\frac{\gamma+1}{\gamma-1} v_{0} v_{1}-v^{2}\right], \quad \operatorname{Pr}=3 / 4,
$$

and likewise for other thermodynamic quantities. For a very clear discussion, see Ref. [115].

To obtain the velocity itself in the approach of Ref. [114] requires the evaluation of an integral involving the specific choice of dynamic viscosity $\eta(\rho, T)$ as a function of $\rho$ and $T$. This generally yields the velocity field in the implicit form $x(v)$. As pointed out by Johnson (2014), some choices of $\eta(\rho, T)$ permit one to invert the relation $x(v)$ to an explicit form $v(x)$. It turns out, however, that to evaluate the infinite Reynolds-number or Péclet-number limits, we need only the Bernoulli relation (A9) of Ref. [114] and its alternative forms (A11) and (A12). We furthermore need one additional constraint, which follows from the constancy of momentum flux:

$$
j_{*} v+p-\eta \partial_{x} v \equiv \tau_{*}
$$

The constant value $\tau_{*}$ can be evaluated far from the shock where the gradient vanishes, giving

$$
\tau_{*}=j_{*} v_{i}+p_{i}, \quad i=0,1
$$

or, using Eq. (A8), from the Rankine-Hugoniot conditions,

$$
\tau_{*}=j_{*} v_{\mathrm{av}}+p_{\mathrm{av}}=\frac{1+\gamma}{\gamma} j_{*} v_{\mathrm{av}} .
$$

Equations (A13) and (A15) and the Bernoulli relation (A11) for $p$ allow us to determine $\eta \partial_{x} v$ in terms of $v$ itself, yielding identical results for any choice of viscosity $\eta(\rho, T)$. As a consequence, all of our ideal limit results are independent of the details of the molecular transport coefficients, apart from the requirement that $\operatorname{Pr}=3 / 4$. Many inertial-range limit results hold with complete generality for all dissipative planar shocks in an ideal gas and do not even depend upon the Prandtl number Pr. Some inertial-range quantities do depend upon $\operatorname{Pr}$, which we can explicitly verify for the cases $\operatorname{Pr}=\infty(\kappa=0)$ and $\operatorname{Pr}=0$ $(\eta=0)$. As was noted by Ref. [169], there are also Bernoulli-type relations for those cases, which yield expressions for the pressure of the form

$p=j_{*}\left[-\frac{1-\gamma}{2} v+\frac{1-\gamma^{2}}{\gamma} v_{\mathrm{av}}+\frac{1+\gamma}{2} \frac{v_{0} v_{1}}{v}\right], \quad \operatorname{Pr}=\infty$,

and

$$
p=j_{*}\left[\frac{1+\gamma}{\gamma} v_{\text {av }}-v\right], \quad \operatorname{Pr}=0 .
$$

Employing these expressions for $p$ and Eqs. (A13) and (A15), we can also obtain formulas for $\eta \partial_{x} v$ with $\operatorname{Pr}=0$, $\infty$, which allow us to extend all of our results for $\operatorname{Pr}=3 / 4$ to those cases. Because the mathematical methods are essentially the same for all three cases, below we discuss explicitly only $\operatorname{Pr}=3 / 4$ and then just briefly mention some corresponding results for $\operatorname{Pr}=0, \infty$.

The solutions of Becker [114] and Johnson [115,169] are a nice example for our general mathematical framework since they converge in $L^{p}$ norms for any $p \in[1, \infty)$ to a weak shock solution of 1D compressible Euler as $\nu \rightarrow 0$. Here, we derive all of the source terms that appear in the kinetic energy and the entropy balance for the shock solutions in the distributional limit as $\eta, \kappa \rightarrow 0$ for the fine-grained balances and as $\ell \rightarrow 0$ for the coarse-grained balances. A fact that we use frequently for ideal stepfunction fields below is

$$
\bar{f}(x)=f_{0}+(\Delta f) \bar{\theta}(x), \quad \bar{g}(x)=g_{0}+(\Delta g) \bar{\theta}(x)
$$

and thus 


$$
\bar{g}=g_{0}+\frac{\Delta g}{\Delta f}\left(\bar{f}-f_{0}\right), \quad \partial_{x} \bar{g}=\frac{\Delta g}{\Delta f} \partial_{x} \bar{f} .
$$

Furthermore,

$$
\partial_{x} \bar{f}(x)=(\Delta f) \bar{\delta}(x)
$$

Similar results can be obtained from

$$
\begin{aligned}
& \bar{f}(x)=f_{\mathrm{av}}+\frac{1}{2}(\Delta f) \overline{\operatorname{sign}}(x), \\
& \bar{g}(x)=g_{\mathrm{av}}+\frac{1}{2}(\Delta g) \overline{\operatorname{sign}}(x) .
\end{aligned}
$$

These relations are very helpful to derive inertial-range expressions for the shock solution.

\section{Kinetic-energy balance}

\section{a. Viscous dissipation}

Using in Eq. (A13) expression (A11) for $p$ with $\operatorname{Pr}=3 / 4$,

$$
\eta\left(\partial_{x} v\right)=\frac{\gamma+1}{2 \gamma} j_{*}\left[v-2 v_{\mathrm{av}}+\frac{v_{0} v_{1}}{v}\right] .
$$

Hence,

$$
\begin{aligned}
\eta\left(\partial_{x} v\right)^{2}= & \frac{\gamma+1}{2 \gamma} j_{*}\left[\partial_{x}\left(\frac{1}{2} v^{2}\right)\right. \\
& \left.-2 v_{\text {av }} \partial_{x} v+v_{0} v_{1} \partial_{x}(\ln v)\right]
\end{aligned}
$$

Since

$$
\begin{aligned}
\mathcal{D}- & \lim _{\eta, \kappa \rightarrow 0}\left[\partial_{x}\left(\frac{1}{2} v^{2}\right), \partial_{x} v, \partial_{x}(\ln v)\right] \\
= & {\left[\frac{1}{2}\left(v_{1}^{2}-v_{0}^{2}\right), \Delta v, \ln \left(v_{1} / v_{0}\right)\right] \delta(x), }
\end{aligned}
$$

one easily obtains

$$
\begin{aligned}
Q_{\text {visc }} & \equiv \mathcal{D}-\lim _{\eta, \kappa \rightarrow 0} \eta\left(\partial_{x} v\right)^{2} \\
& =\frac{\gamma+1}{2 \gamma} j_{*}\left[v_{1} v_{0} \ln \left(\frac{v_{1}}{v_{0}}\right)-\frac{1}{2}\left(v_{1}^{2}-v_{0}^{2}\right)\right] \delta(x) .
\end{aligned}
$$

Note that $Q_{\text {visc }} \geq 0$ because

$$
f(\theta)=\theta \ln \theta-\frac{1}{2} \theta^{2}+\frac{1}{2}>0, \quad 0 \leq \theta<1
$$

and $f(1)=0$. This result is Prandtl-number dependent. In fact, $Q_{\text {visc }}$ for $\operatorname{Pr}=\infty$ is larger by a factor of $\gamma$, and for $\operatorname{Pr}=0$, obviously, $Q_{\text {visc }}=0$.

\section{b. Pressure-dilatation defect}

From Eq. (A11) for $p$ with $\operatorname{Pr}=3 / 4$, we have

Thus,

$$
\begin{aligned}
p * \Theta & \equiv \mathcal{D}-\lim _{\eta, \kappa \rightarrow 0} p\left(\partial_{x} v\right) \\
& =\frac{j_{*}}{2 \gamma}\left[(\gamma+1) v_{1} v_{0} \ln \left(\frac{v_{1}}{v_{0}}\right)-\frac{1}{2}(\gamma-1)\left(v_{1}^{2}-v_{0}^{2}\right)\right] \delta(x) .
\end{aligned}
$$

This result is also Prandtl-number dependent (see below), and the above expression holds only for $\operatorname{Pr}=3 / 4$.

Next, we calculate $p \circ \Theta$. Since $v$ and $p$ in the ideal limit are both step functions, $\partial_{x} \bar{v}=(\Delta v / \Delta p) \partial_{x} \bar{p}$, so

$$
\bar{p} \partial_{x} \bar{v}=\frac{\Delta v}{\Delta p} \partial_{x}\left(\frac{1}{2} \bar{p}^{2}\right)
$$

and thus

$$
\begin{aligned}
p \circ \Theta & \equiv \mathcal{D}-\lim _{\ell \rightarrow 0} \bar{p}\left(\partial_{x} \bar{v}\right) \\
& =(\Delta v) p_{\mathrm{av}} \delta(x)=\frac{j_{*}}{2 \gamma}\left(v_{1}^{2}-v_{0}^{2}\right) \delta(x)
\end{aligned}
$$

using $p_{\text {av }}=j_{*} v_{\text {av }} / \gamma$ from Eq. (A8). Note that this result is independent of the particular choice of filter kernel $G$, as required. It is also completely independent of the molecular dissipation, as it is determined solely from the limiting Euler solution fields. One finds, by subtracting, that

$$
\begin{aligned}
\tau(p, \Theta) & \equiv p * \Theta-p \circ \Theta \\
& =\frac{\gamma+1}{2 \gamma} j_{*}\left[v_{1} v_{0} \ln \left(\frac{v_{1}}{v_{0}}\right)-\frac{1}{2}\left(v_{1}^{2}-v_{0}^{2}\right)\right] \delta(x) .
\end{aligned}
$$

Clearly, $Q_{\text {visc }}=\tau(p, \Theta)$ for $\operatorname{Pr}=3 / 4$. This same identity, in fact, holds for all values of the Prandtl number, allowing us to infer the $\operatorname{Pr}$ dependence of $p * \Theta$ from that of $Q_{\text {visc }}$. The underlying reason for this identity, which is valid for all planar shocks in an ideal gas, is explained in the next subsection on kineticenergy flux. 


\section{c. Kinetic-energy flux}

Baropycnal work.-Using $\bar{\tau}(\rho, v)=\overline{\rho v}-\bar{\rho} \bar{v}=j_{*}-\bar{\rho} \bar{v}$,

$$
\frac{1}{\bar{\rho}} \bar{\tau}(\rho, v)=\frac{j_{*}}{\bar{\rho}}-\bar{v} .
$$

Using $\partial_{x} \bar{p}=(\Delta p / \Delta \rho) \partial_{x} \bar{\rho}=(\Delta p / \Delta v) \partial_{x} \bar{v}$,

$$
\frac{1}{\bar{\rho}} \bar{\tau}(\rho, v) \partial_{x} \bar{p}=j_{*} \frac{\Delta p}{\Delta \rho} \partial_{x}(\ln \bar{\rho})-\frac{\Delta p}{\Delta v} \partial_{x}\left(\frac{1}{2} \bar{v}^{2}\right) .
$$

Thus,

$$
\begin{aligned}
Q_{\text {baro }} & \equiv \mathcal{D}-\lim _{\ell \rightarrow 0} \frac{\partial_{x} \bar{p}}{\bar{\rho}} \bar{\tau}(\rho, v) \\
& =\Delta p\left[\frac{j_{*}}{\Delta \rho} \ln \left(\frac{\rho_{1}}{\rho_{0}}\right)-v_{\mathrm{av}}\right] \delta(x) \\
& =-j_{*}\left[v_{1} v_{0} \ln \left(\frac{v_{1}}{v_{0}}\right)-\frac{1}{2}\left(v_{1}^{2}-v_{0}^{2}\right)\right] \delta(x),
\end{aligned}
$$

where the final line was obtained using $\Delta p=-j_{*} \Delta v$, which follows either from Eq. (A8) or directly from the Rankine-Hugoniot conditions (A6). Note that $Q_{\text {baro }} \leq 0$.

We see again that the limiting inertial-range result $Q_{\text {baro }}$ is independent of the filter kernel $G$. This is true for all of the limits as $\ell \rightarrow 0$ of inertial-range expressions for the shock solutions that we obtain in this appendix. Thus, we make no further note of this fact for the other limits derived below. Note that $Q_{\text {baro }}$ is also completely independent of the molecular transport coefficients, as are all other quantities that are determined solely by the limiting Euler solution fields.

Deformation work.—Using $\tilde{v}=\overline{\rho v} / \bar{\rho}=j_{*} / \bar{\rho}$,

$$
\bar{\rho} \partial_{x} \tilde{v}=-\frac{j_{*}}{\bar{\rho}} \partial_{x} \bar{\rho} .
$$

Likewise, from its definition and $\rho v=j_{*}$, one gets

$$
\begin{aligned}
\tilde{\tau}(v, v) & =\frac{j_{*} \bar{v}}{\bar{\rho}}-\frac{j_{*}^{2}}{\bar{\rho}^{2}} \\
& =j_{*}\left[\frac{\Delta v}{\Delta \rho}+\left(v_{\mathrm{av}}-\frac{\Delta v}{\Delta \rho} \rho_{\mathrm{av}}\right) \frac{1}{\bar{\rho}}-\frac{j_{*}}{\bar{\rho}^{2}}\right]
\end{aligned}
$$

after substituting $\bar{v}=v_{\mathrm{av}}+\frac{\Delta v}{\Delta \rho}\left(\bar{\rho}-\rho_{\mathrm{av}}\right)$ from Eq. (A21). Thus,

$$
\begin{aligned}
Q_{\text {defor }} \equiv & -\mathcal{D}-\lim _{\ell \rightarrow 0} \bar{\rho}\left(\partial_{x} \tilde{v}\right) \tilde{\tau}(v, v) \\
= & j_{*}^{2}\left[\frac{\Delta v}{\Delta \rho} \Delta(\ln \rho)-\left(v_{\mathrm{av}}-\frac{\Delta v}{\Delta \rho} \rho_{\mathrm{av}}\right) \Delta\left(\frac{1}{\rho}\right)\right. \\
& \left.+j_{*} \Delta\left(\frac{1}{2 \rho^{2}}\right)\right] \delta(x) \\
= & j_{*}\left[v_{1} v_{0} \ln \left(\frac{v_{1}}{v_{0}}\right)-\frac{1}{2}\left(v_{1}^{2}-v_{0}^{2}\right)\right] \delta(x) \geq 0
\end{aligned}
$$

upon simplification. Thus, $Q_{\text {flux }}=Q_{\text {baro }}+Q_{\text {defor }}=0$. Since $Q_{\text {visc }}=\tau(p, \Theta)+Q_{\text {flux }}$ in general, this explains why $Q_{\text {visc }}=\tau(p, \Theta)$ holds independent of the molecular transport coefficients for any planar ideal-gas shock. Note that the last identity can also be restated as $-p * \Theta+$ $Q_{\text {visc }}=-p \circ \Theta$, which corroborates, for these solutions, the general argument in the text that the sum of $-p * \Theta$ and $Q_{\text {visc }}$ should be completely independent of the molecular dissipation, even though separately the two terms are Prandtl-number dependent.

In physical terms, there is a loss of kinetic energy $-p \circ \Theta$ at the shock and an equal gain $-p * \Theta+Q_{\text {visc }}$ of internal energy. There is no external force to balance the kineticenergy loss and no cooling to balance the internal energy gain. While these shock solutions are stationary, they are not, however, homogeneous or isotropic. Thus, the loss or gain is balanced by space transport of kinetic or internal energy into or away from the shock, respectively. For example, the space flux of kinetic energy is

$$
J_{\text {kin }}=\left(\frac{1}{2} \rho v^{2}+p\right) v=j_{*}\left(\frac{1}{2} v^{2}+\frac{p}{\rho}\right) .
$$

One readily finds from Eq. (A8) for $p_{i}, i=0,1$ that

$$
\Delta J_{\text {kin }}=\frac{j_{*}}{2 \gamma}\left(v_{1}^{2}-v_{0}^{2}\right)<0,
$$

so more kinetic energy enters the shock than leaves it, and the difference is exactly the correct amount to offset the loss due to pressure work. Similarly, more internal energy is transported away from the shock than what enters it, balancing the gain from pressure work and heating. This follows directly from the conservation of total energy or by using $J_{\mathrm{int}}=u v$ for space flux of internal energy and evaluating $\Delta J_{\text {int }}=-\Delta J_{\text {kin }}$.

\section{Entropy balance \\ a. Dissipation range}

Viscous heating $\beta * Q_{\text {visc }}$ - - Using Eq. (A22) for $\eta \partial_{x} v$, we write 


$$
\begin{aligned}
\frac{\eta\left(\partial_{x} v\right)^{2}}{T} & =\frac{\gamma+1}{2 \gamma} j_{*}\left[v-2 v_{\mathrm{av}}+\frac{v_{0} v_{1}}{v}\right] \frac{\partial_{x} v}{T} \\
& =\frac{\gamma+1}{2 \gamma} j_{*}\left(1+\frac{v_{0} v_{1}}{v^{2}}\right) \frac{v \partial_{x} v}{T}-\frac{\gamma+1}{\gamma} j_{*} v_{\mathrm{av}} \frac{\partial_{x} v}{T} .
\end{aligned}
$$

In the first term, we replace $v^{2}$ with $T$ using both Eq. (A12) and its derivative $v \partial_{x} v=-c_{P} \partial_{x} T$, while in the second term, we replace $T$ with $v^{2}$ using Eq. (A12). Elementary antiderivatives give, for the first term,

$-j_{*} \gamma c_{V} \partial_{x}(\ln T)+\frac{1}{2} j_{*}(\gamma-1) c_{V} \partial_{x} \ln \left|a^{2}-2 c_{P} T\right|$,

with $a^{2}=[(\gamma+1) /(\gamma-1)] v_{0} v_{1}$, and for the second term,

$$
-2 j_{*} c_{V}(\gamma+1) \frac{v_{a v}}{a} \partial_{x} \operatorname{arctanh}(v / a) .
$$

Noting that $a^{2}-2 c_{P} T=v^{2}=j_{*}^{2} / \rho^{2}$, we finally get

$$
\begin{aligned}
\beta * Q_{\mathrm{visc}}= & \mathcal{D}-\lim _{\eta, \kappa \rightarrow 0} \frac{\eta\left(\partial_{x} v\right)^{2}}{T} \\
= & -j_{*} c_{V}\left[\gamma \ln \left(\frac{T_{1}}{T_{0}}\right)+(\gamma-1) \ln \left(\frac{\rho_{1}}{\rho_{0}}\right)\right. \\
& \left.+2(\gamma+1) \frac{v_{a v}}{a} \Delta(\operatorname{arctanh}(v / a))\right] \delta(x) .
\end{aligned}
$$

This expression holds only for $\operatorname{Pr}=3 / 4$, and the quantity is generally Prandtl-number dependent. For example, $\beta *$ $Q_{\text {visc }}=0$ for $\operatorname{Pr}=0$.

Thermal conduction $\Sigma_{\text {therm. }}$ - -Note that, since $\operatorname{Pr}=3 / 4$ and $v \partial_{x} v=-c_{P} \partial_{x} T$,

$$
\kappa \partial_{x} T=-\eta v \partial_{x} v
$$

and thus, also using Eq. (A22) for $\eta \partial_{x} v$,

$$
\begin{aligned}
\kappa \partial_{x} T & =-j_{*} \frac{\gamma+1}{2 \gamma}\left(v^{2}-2 v_{\mathrm{av}} v+v_{0} v_{1}\right) \\
& =-j_{*}(\gamma+1)\left(\frac{v_{0} v_{1}}{\gamma-1}-c_{V} T\right)+j_{*} \frac{\gamma+1}{\gamma} v_{\mathrm{av}} v .
\end{aligned}
$$

Hence,

$$
\begin{aligned}
\frac{\kappa\left(\partial_{x} T\right)^{2}}{T^{2}}= & -j_{*}(\gamma+1)\left(\frac{v_{0} v_{1}}{\gamma-1}-c_{V} T\right) \frac{\partial_{x} T}{T^{2}} \\
& -4 j_{*} c_{V}(\gamma+1) v_{\mathrm{av}} \frac{v^{2} \partial_{x} v}{\left(a^{2}-v^{2}\right)^{2}} .
\end{aligned}
$$

Elementary antiderivatives and some lengthy algebraic simplifications give

$$
\begin{aligned}
\frac{\kappa\left(\partial_{x} T\right)^{2}}{T^{2}}= & j_{*} \frac{\gamma+1}{\gamma-1} v_{0} v_{1} \partial_{x}\left(\frac{1}{T}\right)+j_{*} c_{V}(\gamma+1) \partial_{x}(\ln T) \\
& -j_{*} \frac{\gamma+1}{\gamma} v_{\mathrm{av}} \partial_{x}\left(\frac{v}{T}\right) \\
& +2 j_{*} c_{V}(\gamma+1) \frac{v_{\mathrm{av}}}{a} \partial_{x} \operatorname{arctanh}(v / a)
\end{aligned}
$$

Taking the limit $\eta, \kappa \rightarrow 0$, one finds, using the Bernoulli relation, that the contributions of the first and third terms in the above expression cancel, giving the final result

$$
\begin{aligned}
\Sigma_{\text {therm }}= & \mathcal{D}-\lim _{\eta, \kappa \rightarrow 0} \frac{\kappa\left(\partial_{x} T\right)^{2}}{T^{2}} \\
= & j_{*} c_{V}(\gamma+1)\left[\ln \left(\frac{T_{1}}{T_{0}}\right)+2 \frac{v_{a v}}{a} \Delta(\operatorname{arctanh}(v / a))\right] \\
& \times \delta(x) .
\end{aligned}
$$

Once again, this quantity is Prandtl-number dependent, and the above expression holds only for $\operatorname{Pr}=3 / 4$. Obviously, $\Sigma_{\text {therm }}=0$ for $\operatorname{Pr}=\infty$.

Total entropy production $\Sigma_{\text {diss }}$. - The inverse hyperbolic tangent terms cancel when added, giving

$$
\begin{aligned}
\Sigma_{\text {diss }} & =\Sigma_{\text {therm }}+\beta * Q_{\text {visc }} \\
& =j_{*} c_{V}\left[\ln \left(\frac{T_{1}}{T_{0}}\right)-(\gamma-1) \ln \left(\frac{\rho_{1}}{\rho_{0}}\right)\right] \delta(x) \\
& =j_{*} \Delta s_{m} \delta(x),
\end{aligned}
$$

using $s_{m}=c_{V} \ln \left(T / C \rho^{\gamma-1}\right)$. We see that $\Sigma_{\text {diss }}>0$ since $\Delta s_{m}>0$ is the standard entropy condition for a Euler shock. The result (A49) could have been anticipated on the basis of simple entropy balance since $J_{\text {ent }}=s v=s_{m} j_{*}$ is the space flux of entropy and $\Delta J_{\text {ent }}=\left(\Delta s_{m}\right) j_{*}$ is the net entropy transported away from the shock. Thus, the entropy production at the shock is balanced by transport of entropy to infinity. The result (A49) for $\Sigma_{\text {diss }}$ is, for this reason, completely independent of the molecular dissipation. Note that $\Sigma_{\text {therm }}=\Sigma_{\text {diss }}$ for $\operatorname{Pr}=0$ and that $\beta * Q_{\text {visc }}=\Sigma_{\text {diss }}$ for $\operatorname{Pr}=\infty$. These results for $\operatorname{Pr}=0, \infty$ can also be obtained by using the Bernoulli-type relations in Refs. $[115,169]$ and by calculating in the same manner as for $\operatorname{Pr}=3 / 4$ above.

\section{b. Inertial range}

Inertial-range viscous heating $\beta \circ Q_{\text {visc }}$ - -From Eq. (A25), $Q_{\text {visc }}=q_{*} \delta(x)$, so

$$
\bar{Q}_{\mathrm{visc}}=q_{*} \bar{\delta}(x) .
$$

On the other hand, for an ideal gas, by definition of $\underline{\beta}$,

$$
\underline{\beta}=c_{V} \frac{\bar{\rho}}{\bar{u}} .
$$


Because $u, \rho$ are step functions in the ideal limit,

$$
\bar{\rho}=\left(\rho_{0}-\frac{\Delta \rho}{\Delta u} u_{0}\right)+\frac{\Delta \rho}{\Delta u} \bar{u}, \quad \bar{\delta}=\frac{\partial_{x} \bar{u}}{\Delta u} .
$$

Thus,

$$
\begin{aligned}
\underline{\beta} \bar{Q}_{\mathrm{visc}} & =c_{V} q_{*}\left[\left(\rho_{0}-\frac{\Delta \rho}{\Delta u} u_{0}\right) \frac{\partial_{x} \bar{u}}{\bar{u} \Delta u} \cdot+\frac{\Delta \rho}{\Delta u} \bar{\delta}(x)\right] \\
& =c_{V} q_{*}\left[\left(\frac{\rho_{0} \Delta u-u_{0} \Delta \rho}{(\Delta u)^{2}}\right) \partial_{x}(\ln \bar{u})+\frac{\Delta \rho}{\Delta u} \bar{\delta}(x)\right] .
\end{aligned}
$$

Hence,

$$
\beta \circ Q_{\mathrm{visc}}=\mathcal{D}-\lim _{\ell \rightarrow 0} \underline{\beta} \bar{Q}_{\mathrm{visc}}=\beta_{*} q_{*} \delta(x),
$$

with

$$
\beta_{*} \equiv c_{V}\left[\left(\frac{\rho_{0} u_{1}-u_{0} \rho_{1}}{(\Delta u)^{2}}\right) \ln \left(\frac{u_{1}}{u_{0}}\right)+\frac{\Delta \rho}{\Delta u}\right] .
$$

This result is obviously independent of the filter kernel $G$ (as are all such limits of coarse-grained quantities), but the quantity $q_{*}$ gives a Prandtl-number dependence.

Pressure-dilatation defect $\beta \circ \tau(p, \Theta)$.-Because of our earlier result $p * \Theta=q_{P V} \delta(x)$, the same argument as above shows that

$$
\mathcal{D}-\lim _{\ell \rightarrow 0} \underline{\beta} \overline{p * \Theta}=\beta_{*} q_{P V} \delta(x) .
$$

Next note that, using $\bar{p}=(\gamma-1) \bar{u}$ and Eq. (A51) for $\underline{\beta}$,

$\underline{\beta} \bar{p} \partial_{x} \bar{v}=c_{V}(\gamma-1) \bar{\rho} \partial_{x} \bar{v}=c_{V}(\gamma-1) \frac{\Delta v}{\Delta \rho} \partial_{x}\left(\frac{1}{2} \bar{\rho}^{2}\right)$,

where the last equality follows from $\partial_{x} \bar{v}=(\Delta v / \Delta \rho) \partial_{x} \bar{\rho}$. Thus,

$$
\begin{aligned}
\mathcal{D}-\lim _{\ell \rightarrow 0} \underline{\beta} \bar{p} \partial_{x} \bar{v} & =c_{V}(\gamma-1)(\Delta v) \rho_{\mathrm{av}} \delta(x) \\
& =c_{V} j_{*}(\gamma-1) \frac{(\Delta v) v_{\mathrm{av}}}{v_{1} v_{0}} \delta(x)
\end{aligned}
$$

after using $\rho=j_{*} / v$. Finally,

$\beta \circ \tau(p, \Theta)=\left[\beta_{*} q_{\mathrm{PV}}-c_{V} j_{*}(\gamma-1) \frac{(\Delta v) v_{\mathrm{av}}}{v_{1} v_{0}}\right] \delta(x)$.

This quantity is, of course, Prandtl-number dependent through the coefficient $q_{\mathrm{PV}}$.

Combined contribution $\beta \circ Q-\beta \circ \tau(p, \Theta)$.- Using the expression (A25) for $q_{*}$ and Eq. (A28) for $q_{\mathrm{PV}}$, one can see that the log term cancels in the difference and

$$
q_{*}-q_{\mathrm{PV}}=-\frac{j_{*}}{\gamma} \Delta\left(\frac{1}{2} v^{2}\right)=j_{*} c_{V}(\Delta T)
$$

In accordance with our earlier remarks, this is the same as the coefficient of $-p \circ \Theta$ and is completely independent of the choice of molecular transport coefficients. Thus,

$$
\beta \circ Q-\beta \circ \tau(p, \Theta)=c_{V} j_{*}\left[\beta_{*}(\Delta T)+(\gamma-1) \frac{v_{\mathrm{av}} \Delta v}{v_{0} v_{1}}\right] \delta(x)
$$

and is also independent of molecular dissipation.

Negentropy flux $\Sigma_{\text {flux }}$. - We first consider the contribution from $\left(\partial_{x} \underline{\beta}\right) \overline{u v}$. From $\underline{\beta}=c_{V} \bar{\rho} / \bar{u}$, one gets

$$
\partial_{x} \underline{\beta}=c_{V}\left(\frac{1}{\bar{u}} \partial_{x} \bar{\rho}-\frac{\bar{\rho}}{\bar{u}^{2}} \partial_{x} \bar{u}\right),
$$

whereas

$$
\overline{u v}=c_{V} \overline{\rho T v}=c_{V} j_{*} \bar{T} .
$$

Writing $\bar{\rho}$ in terms of $\bar{u}$ using Eq. (A19) and a similar relation for $\bar{T}$ in terms of $\bar{u}$, one finds, after some simplifications,

$$
\begin{aligned}
\left(\partial_{x} \underline{\beta}\right) \overline{u v}= & -c_{V}^{2} j_{*}\left(\rho_{0}-\frac{\Delta \rho}{\Delta u} u_{0}\right) \\
& \times\left[\left(T_{0}-\frac{\Delta T}{\Delta u} u_{0}\right) \frac{\partial_{x} \bar{u}}{\bar{u}^{2}}+\frac{\Delta T}{\Delta u} \frac{\partial_{x} \bar{u}}{\bar{u}}\right] .
\end{aligned}
$$

Thus,

$$
\begin{aligned}
\mathcal{D}-\lim _{\ell \rightarrow 0}\left(\partial_{x} \underline{\beta}\right) \overline{u v} \\
=-c_{V}^{2} j_{*}\left(\rho_{0}-\frac{\Delta \rho}{\Delta u} u_{0}\right) \\
\quad \times\left[\left(T_{0}-\frac{\Delta T}{\Delta u} u_{0}\right) \frac{\Delta u}{u_{0} u_{1}}+\frac{\Delta T}{\Delta u} \ln \left(\frac{u_{1}}{u_{0}}\right)\right] \delta(x) .
\end{aligned}
$$

Using the relation

$\left(T_{0} u_{1}-T_{1} u_{0}\right)\left(\rho_{0} u_{1}-\rho_{1} u_{0}\right)=(\Delta T)(\Delta \rho) u_{0} u_{1}$,

which follows from $u=c_{V} \rho T$, and the definition of $\beta_{*}$ from Eq. (A55), this reduces to

$$
\mathcal{D}-\lim _{\ell \rightarrow 0} \partial_{x} \underline{\beta} \cdot \overline{u v}=-\beta_{*} c_{V}(\Delta T) j_{*} \delta(x) .
$$

Next, note that $\partial_{x} \underline{\lambda}_{m} \cdot \overline{\rho v}=j_{*} \partial_{x} \underline{\lambda}_{m}$. Hence,

$$
\mathcal{D}-\lim _{\ell \rightarrow 0} \partial_{x} \underline{\lambda}_{m} \cdot \overline{\rho v}=\left(\Delta \lambda_{m}\right) j_{*} \delta(x) .
$$

For any equation of state, the Gibbs fundamental relation may be written as $s_{m}=h_{m} / T-\lambda_{m}$. For an ideal gas $h_{m}=c_{P} T$, so $s_{m}=c_{P}-\lambda_{m}$ and $\Delta s_{m}=-\left(\Delta \lambda_{m}\right)$. Thus, 


$$
\mathcal{D}-\lim _{\ell \rightarrow 0} \partial_{x} \underline{\lambda}_{m} \cdot \overline{\rho v}=-\left(\Delta s_{m}\right) j_{*} \delta(x) .
$$

Finally, consider the contributions from $\partial_{x} \underline{\beta}(\bar{u} \cdot \bar{v})$, $\partial_{x} \underline{\lambda}_{m}(\bar{\rho} \cdot \bar{v})$. Using Eq. (A62) for $\partial_{x} \underline{\beta}$, one has

$$
\partial_{x} \underline{\beta}(\bar{u} \cdot \bar{v})=c_{V} \bar{v}\left(\partial_{x} \bar{\rho}\right)-c_{V} \frac{\bar{\rho} \bar{v}}{\bar{u}}\left(\partial_{x} \bar{u}\right) .
$$

Next, use $\partial_{x} \underline{\lambda}_{m}=-\partial_{x} \underline{s}_{m}$ and $s_{m}=c_{V} \ln \left(u / C \rho^{\gamma}\right)$ to obtain

$$
\partial_{x} \underline{\lambda}_{m}(\bar{\rho} \cdot \bar{v})=c_{V} \gamma \bar{v}\left(\partial_{x} \bar{\rho}\right)-c_{V} \frac{\bar{\rho} \bar{v}}{\bar{u}}\left(\partial_{x} \bar{u}\right) .
$$

Subtracting these two expressions gives

$$
\begin{aligned}
& \partial_{x} \underline{\beta}(\bar{u} \cdot \bar{v})-\partial_{x} \underline{\lambda}_{m}(\bar{\rho} \cdot \bar{v}) \\
& \quad=c_{V}(\gamma-1) \bar{v}\left(\partial_{x} \bar{\rho}\right)=(\gamma-1) c_{V} \frac{\Delta \rho}{\Delta v} \bar{v}\left(\partial_{x} \bar{v}\right) .
\end{aligned}
$$

From this result, it follows easily that

$$
\begin{gathered}
\mathcal{D}-\lim _{\ell \rightarrow 0}\left[\partial_{x} \underline{\beta}(\bar{u} \cdot \bar{v})-\partial_{x} \underline{\lambda}_{m}(\bar{\rho} \cdot \bar{v})\right] \\
=c_{V}(\gamma-1) j_{*} \frac{v_{a v} \Delta v}{v_{0} v_{1}} \delta(x) .
\end{gathered}
$$

Putting together all of these results,

$$
\begin{aligned}
\Sigma_{\text {flux }} & =\mathcal{D}-\lim _{\ell \rightarrow 0}\left[\partial_{x} \underline{\beta} \bar{\tau}(u, v)-\partial_{x} \underline{\lambda}_{m} \bar{\tau}(\rho, v)\right] \\
& =j_{*}\left[\Delta s_{m}-c_{V} \beta_{*}(\Delta T)-c_{V}(\gamma-1) \frac{v_{a v} \Delta v}{v_{0} v_{1}}\right] \delta(x),
\end{aligned}
$$

which is valid, in general, for the limiting Euler solution independent of the microscopic dissipation mechanism. Note that the first and third terms in the square brackets of the last expression are positive, while the second term is negative. We do not present details here, but it is possible to show that the sum of all three terms is strictly positive as a function of compression factor $R=\rho_{1} / \rho_{0}=v_{0} / v_{1}$ and maximum compression factor $R_{\infty}=(\gamma+1) /(\gamma-1)$ over the allowed range $1 \leq R \leq R_{\infty}$.

Total entropy production $\Sigma_{\text {inert }}$ - - For the final inertialrange entropy production, we get from Eqs. (A61) and (A74) that

$$
\Sigma_{\text {inert }}=\Sigma_{\text {flux }}+\beta \circ(Q-\tau(p, \Theta))=j_{*} \Delta s_{m} \delta(x),
$$

which is independent of the molecular dissipation and in exact agreement with the net result of the dissipation-range/ fine-grained calculation in Appendix A 3 a. From relation (92), it is also true that

$$
\Sigma_{\text {flux } *}=\Sigma_{\text {flux }}+\beta \circ(Q-\tau(p, \Theta))=j_{*} \Delta s_{m} \delta(x) .
$$

The intrinsic negentropy flux consistently gives the net entropy production for this problem since $I_{\text {flux }}=0$ for an ideal-gas equation of state.

\section{APPENDIX B: NO-GO THEOREM FOR ASYMPTOTIC VALIDITY OF BAROTROPIC MODELS}

We present here an argument that barotropic models cannot become exact in the limit of any parameter $\epsilon \rightarrow 0$ (analogous to Mach number for incompressible fluids) unless the entropy production also vanishes in the same limit. Since barotropic models contain no thermal conductivity, we assume for simplicity that the fluid has infinite Prandtl number, or $\kappa=0$. In that case, the entropy balance (19) for compressible Navier-Stokes reduces to the equation for $s_{m}$, the entropy per mass:

$$
\rho D_{t} s_{m}=\Sigma \geq 0
$$

with $\Sigma=Q_{\text {visc }} / T$. In fact, our argument applies to any other fluid model for which Eq. (B1) holds. We also assume that the equation of state $p=p\left(\rho, s_{m}\right)$ satisfies $\left(\partial p / \partial s_{m}\right)_{\rho}>0$. By a Maxwell relation, $\left(\partial p / \partial s_{m}\right)_{\rho}=$ $-(\partial T / \partial v)_{s_{m}}$, this is equivalent to the condition that the fluid cools during adiabatic expansion, which holds true for most fluids (including ideal gases). We then consider any sequence of flows $\left(\mathbf{v}^{\epsilon}, \rho^{\epsilon}, s_{m}^{\epsilon}\right)$ for which the barotropic closure $p=p_{B}(\rho)$ becomes asymptotically exact as $\epsilon \rightarrow 0$, in the sense that

$$
\begin{gathered}
p\left(\rho^{\epsilon}, s_{m}^{\epsilon}\right)-p_{B}\left(\rho^{\epsilon}\right) \rightarrow 0, \\
\left(\frac{\partial p}{\partial \rho}\right)_{s_{m}}\left(\rho^{\epsilon}, s_{m}^{\epsilon}\right)-\frac{\partial p_{B}}{\partial \rho}\left(\rho^{\epsilon}\right) \rightarrow 0 .
\end{gathered}
$$

Since the speed of sound is given by $c_{s}^{2}=(\partial p / \partial \rho)_{s_{m}}$, the last condition is equivalent to the requirement that $c_{s e}^{2} \rightarrow c_{s B}^{2}$, where $c_{s B}^{2}=p_{B}^{\prime}(\rho)$ gives the speed of sound in the barotropic closure. Finally, we assume that all of the solution fields $\mathbf{v}^{\epsilon}, \rho^{\epsilon}, s_{m}^{\epsilon}$ and also the divergence $\Theta^{\epsilon}$ are bounded by fixed constants for $\epsilon>0$ and that $\left(\partial p / \partial s_{m}\right)_{\rho}^{\epsilon} \geq \Phi_{0}$, with some fixed constant. For an ideal gas, the last assumption means that vacuum (zero-density) conditions are not approached. For any sequence of flows satisfying these reasonable conditions, we show that entropy production per volume $\Sigma^{\epsilon} \rightarrow 0$ necessarily.

To show this, we consider how $p=p\left(\rho, s_{m}\right)$ and $p_{B}=$ $p_{B}(\rho)$ evolve along fluid particle trajectories. Using Eq. (1) for the mass density in the form $D_{t} \rho=-\rho \Theta$ and Eq. (B1), we find, by the chain rule,

$$
\begin{aligned}
D_{t} p & =-\Theta \rho(\partial p / \partial \rho)+(\Sigma / \rho)\left(\partial p / \partial s_{m}\right), \\
D_{t} p_{B} & =-\Theta \rho\left(\partial p_{B} / \partial \rho\right) .
\end{aligned}
$$


Taking $\mathbf{X}(\mathbf{x}, t)$ to be the Lagrangian fluid trajectory that satisfies $(d / d t) \mathbf{X}=\mathbf{v}(\mathbf{X}, t)$ and that starts at point $\mathbf{x}$, and defining the difference $\Delta\left(\rho, s_{m}\right)=p\left(\rho, s_{m}\right)-p_{B}(\rho)$, one easily obtains, by subtracting the two equations in Eq. (B4) and integrating in time,

$$
\begin{aligned}
& \left.\Delta\right|_{(\mathbf{X}(\mathbf{x}, t), t)}-\left.\Delta\right|_{(\mathbf{x}, 0)} \\
& \quad=\int_{0}^{t} d \tau\left[-\Theta \rho(\partial \Delta / \partial \rho)+(\Sigma / \rho)\left(\partial p / \partial s_{m}\right)\right]_{(\mathbf{X}(\mathbf{x}, \tau), \tau)} .
\end{aligned}
$$

Multiplying this relation by initial density $\rho(\mathbf{x}, 0)$, integrating $\mathbf{x}$ over space, and noting the Jacobian $\operatorname{det}(\partial \mathbf{X} / \partial \mathbf{x})=$ $\rho_{0} / \rho_{t}$ with $\rho_{t}=\rho(\mathbf{x}, t)$, we finally obtain

$$
\begin{aligned}
\int_{0}^{t} d \tau \int d^{d} x \Sigma_{\tau}\left(\partial p / \partial s_{m}\right)_{\tau}= & \int d^{d} x\left[\rho_{t} \Delta_{t}-\rho_{0} \Delta_{0}\right] \\
& +\int_{0}^{t} d \tau \int d^{d} x \Theta_{\tau} \rho_{\tau}^{2}(\partial \Delta / \partial \rho)_{\tau} .
\end{aligned}
$$

This relation clearly implies that $\Sigma^{\epsilon} \rightarrow 0$ for any sequence of flows such that $\Delta^{\epsilon} \rightarrow 0$. This can be shown rigorously from the bound

$$
\begin{aligned}
0 \leq & \Phi_{0} \int_{0}^{t} d \tau \int d^{d} x \Sigma_{\tau}^{\epsilon} \leq \int d^{d} x\left[\rho_{t}^{\epsilon}\left|\Delta_{t}^{\epsilon}\right|+\rho_{0}^{\epsilon}\left|\Delta_{0}^{\epsilon}\right|\right] \\
& +\int_{0}^{t} d \tau \int d^{d} x\left|\Theta_{\tau}^{\epsilon}\right|\left[\rho_{\tau}^{\epsilon}\right]^{2}\left|(\partial \Delta / \partial \rho)_{\tau}^{\epsilon}\right| .
\end{aligned}
$$

For example, if the convergence conditions $\Delta^{\epsilon} \rightarrow 0$ and $\partial \Delta^{\epsilon} / \partial \rho \rightarrow 0$ in Eqs. (B2) and (B3) are taken to hold pointwise in space-time (or even in $L^{1}$-sense) and the fields $\Theta^{\epsilon}, \rho^{\epsilon}$ are bounded uniformly in $\epsilon>0$, then as $\epsilon \rightarrow 0$,

$$
\int_{0}^{t} d \tau \int d^{d} x \Sigma_{\tau}^{\epsilon} \rightarrow 0
$$

This argument illustrates the grave concerns with the physical consistency of viscous barotropic models. It appears quite difficult to make these models consistent with basic physics principles (thermodynamics, conservation of energy, second law) for isolated molecular fluids.

[1] D. Falceta-Goncalves, G. Kowal, E. Falgarone, and A. C.-L. Chian, Turbulence in the Interstellar Medium, Nonlinear Processes Geophys. 21, 587 (2014).

[2] J. Ballesteros-Paredes, R. S. Klessen, M.-M. Mac Low, and E. Vazquez-Semadeni, Molecular Cloud Turbulence and Star Formation, in Protostars Planets, edited by B. Reipurth, D. Jewitt, and K. Keil (University of Arizona Press, Tucson, 2007), pp. 63-80.
[3] C. Federrath, The Role of Turbulence, Magnetic Fields and Feedback for Star Formation, J. Phys. Conf. Ser. 719, 012002 (2016).

[4] B. M. Haines, F. F. Grinstein, L. Welser-Sherrill, and J. R. Fincke, Laser-Driven Turbulence in High Energy Density Physics and Inertial Confinement Fusion Experiments, in Coarse Grained Simulation and Turbulent Mixing, edited by F. F. Grinstein (Cambridge University Press, New York, 2016), pp. 232-281.

[5] M. F. Modest and D. C. Haworth, Chemically Reacting Turbulent Flows, in Radiative Heat Transfer in Turbulent Combustion Systems (Springer, New York, 2016), pp. 13-42.

[6] A. J. Smits and J.-P. Dussauge, Turbulent Shear Layers in Supersonic Flow (Springer Science \& Business Media, New York, 2006).

[7] N. Bucciantini, Review of the Theory of Pulsar-Wind Nebulae, Astron. Nachr. 335, 234 (2014).

[8] R. Narayan and P. Kumar, A Turbulent Model of GammaRay Burst Variability, Mon. Not. R. Astron. Soc. 394, L117 (2009).

[9] R. D. De Souza, T. Koide, and T. Kodama, Hydrodynamic Approaches in Relativistic Heavy Ion Reactions, Prog. Part. Nucl. Phys. 86, 35 (2016).

[10] L. Fritz, J. Schmalian, M. Müller, and S. Sachdev, Quantum Critical Transport in Clean Graphene, Phys. Rev. B 78, 085416 (2008).

[11] A. B. Kashuba, Conductivity of Defectless Graphene, Phys. Rev. B 78, 085415 (2008).

[12] M. Müller, J. Schmalian, and L. Fritz, Graphene: A Nearly Perfect Fluid, Phys. Rev. Lett. 103, 025301 (2009).

[13] C. Hoyos, B. S. Kim, and Y. Oz, Lifshitz Hydrodynamics, J. High Energy Phys. 11 (2013) 145.

[14] R. A. Davison, K. Schalm, and J. Zaanen, Holographic Duality and the Resistivity of Strange Metals, Phys. Rev. B 89, 245116 (2014).

[15] A. N. Kolmogorov, The Local Structure of Turbulence in Incompressible Viscous Fluid for Very Large Reynolds Numbers, Dokl. Akad. Nauk SSSR 30, 9 (1941).

[16] A. N. Kolmogorov, On the Degeneration of Isotropic Turbulence in an Incompressible Viscous Fluid, Dokl. Akad. Nauk SSSR 31, 538 (1941).

[17] A. N. Kolmogorov, Dissipation of Energy in Locally Isotropic Turbulence, Dokl. Akad. Nauk SSSR 32, 16 (1941).

[18] A. M. Obukhov, On the Distribution of Energy in the Spectrum of Turbulent Flow, Dokl. Akad. Nauk SSSR 32, 22 (1941).

[19] L. Onsager, The Distribution of Energy in Turbulence, in Minutes of the Meeting of the Metropolitan Section held at Columbia University, New York, 1945 [Phys. Rev. 68, 281 (1945)].

[20] L. Onsager, Statistical Hydrodynamics, Nuovo Cimento Suppl. 6, 279 (1949).

[21] W. Heisenberg, Zur statistischen Theorie der Turbulenz, Z. Phys. 124, 628 (1948).

[22] G. F. von Weizsacker, Das Spektrum der Turbulenz bei grossen Reynoldschen Zahlen, Z. Phys. 124, 614 (1948).

[23] H. Aluie, Scale Decomposition in Compressible Turbulence, Physica (Amsterdam) 247D, 54 (2013). 
[24] G. Falkovich, I. Fouxon, and Y. Oz, New Relations for Correlation Functions in Navier-Stokes Turbulence, J. Fluid Mech. 644, 465 (2010).

[25] I. Fouxon and Y. Oz, Exact Scaling Relations in Relativistic Hydrodynamic Turbulence, Phys. Lett. B 694, 261 (2010).

[26] G. I. Taylor, Observations and Speculations on the Nature of Turbulent Motion (1917), in The Scientific Papers of Sir Geoffrey Ingram Taylor: Volume 2, Meteorology, Oceanography and Turbulent Flow, Scientific Papers, edited by G. K. Batchelor (Cambridge University Press, Cambridge, England, 1960), pp. 69-78.

[27] H. L. Dryden, A Review of the Statistical Theory of Turbulence, Q. Appl. Math. 1, 7 (1943).

[28] K. R. Sreenivasan, On the Scaling of the Turbulence Energy Dissipation Rate, Phys. Fluids 27, 1048 (1984).

[29] Y. Kaneda, T. Ishihara, M. Yokokawa, K. Itakura, and A. Uno, Energy Dissipation Rate and Energy Spectrum in High Resolution Direct Numerical Simulations of Turbulence in a Periodic Box, Phys. Fluids 15, L21 (2003).

[30] B. R. Pearson, P.-A. Krogstad, and W. Van De Water, Measurements of the Turbulent Energy Dissipation Rate, Phys. Fluids 14, 1288 (2002).

[31] G. L. Eyink and K. R. Sreenivasan, Onsager and the Theory of Hydrodynamic Turbulence, Rev. Mod. Phys. 78, 87 (2006).

[32] It is important to emphasize that this "dissipative anomaly" proposed by Onsager involves no violation of the principle of conservation of energy. Incompressible Euler equations are a (noncanonical) Hamiltonian system whose Hamiltonian function is the kinetic energy. Thus, smooth solutions of incompressible Euler equations conserve kinetic energy as a Noether theorem consequence of time-translation symmetry. It is the formal conservation of kinetic energy that is anomalous for singular solutions of incompressible Euler equations.

[33] A. M. Polyakov, Conformal Turbulence, arXiv:hep-th/ 9209046.

[34] A. M. Polyakov, The Theory of Turbulence in Two Dimensions, Nucl. Phys. B396, 367 (1993).

[35] P. Kestener and A. Arneodo, Generalizing the WaveletBased Multifractal Formalism to Random Vector Fields: Application to Three-Dimensional Turbulence Velocity and Vorticity Data, Phys. Rev. Lett. 93, 044501 (2004).

[36] U. Frisch and G. Parisi, On the Singularity Structure of Fully Developed Turbulence, in Turbulence and Predictability in Geophysical Fluid Dynamics and Climate Dynamics (Elsevier, Amsterdam, North-Holland, 1985), pp. 84-88.

[37] Uriel Frisch, Turbulence: The Legacy of A. N. Kolmogorov (Cambridge University Press, Cambridge, England, 1995).

[38] H. Aluie, Compressible Turbulence: The Cascade and Its Locality, Phys. Rev. Lett. 106, 174502 (2011).

[39] G. L. Eyink, Energy Dissipation without Viscosity in Ideal Hydrodynamics I. Fourier Analysis and Local Energy Transfer, Physica (Amsterdam) 78D, 222 (1994).

[40] G. L. Eyink, Local Energy Flux and the Refined Similarity Hypothesis, J. Stat. Phys. 78, 335 (1995).
[41] P. Constantin, W. E, and E. S. Titi, Onsager's Conjecture on the Energy Conservation for Solutions of Euler's Equation, Commun. Math. Phys. 165, 207 (1994).

[42] J. Duchon and R. Robert, Inertial Energy Dissipation for Weak Solutions of Incompressible Euler and Navier-Stokes Equations, Nonlinearity 13, 249 (2000).

[43] C. De Lellis and L. Székelyhidi, Jr., Continuous Dissipative Euler Flows and a Conjecture of Onsager, in European Congress of Mathematics: Kraków, 2012, edited by R. Latała, A. Ruciński, P. Strzelecki, J. Światkowski, and D. Wrzosek (European Mathematical Society, Zürich, 2013), pp. 13-30.

[44] C. De Lellis and L. Székelyhidi, Jr., The h-Principle and the Equations of Fluid Dynamics, Bull. Am. Math. Soc. 49, 347 (2012).

[45] P. Isett, A Proof of Onsager's Conjecture, arXiv:1608 .08301

[46] E. C. G. Stueckelberg and A. Petermann, La Normalisation des Constantes Dans la Théorie des Quanta, Helv. Phys. Acta 26, 499 (1951).

[47] M. Gell-Mann and F. E. Low, Quantum Electrodynamics at Small Distances, Phys. Rev. 95, 1300 (1954).

[48] N. N. Bogolyubov and D. V. Shirkov, Group of Charge Renormalization in Quantum Field Theory, Dokl. Akad. Nauk SSSR 103, 391 (1955).

[49] D. J. Gross, Applications of the Renormalization Group to High-Energy Physics, in Methods in Field Theory, Les Houches 1975, Session XVIII, edited by R. Balian and J. Zinn-Justin (North-Holland Publishing, Amsterdam, 1976), pp. 141-250.

[50] S. Chandrasekhar, The Fluctuations of Density in Isotropic Turbulence, Proc. R. Soc. A 210, 18 (1951).

[51] R. H. Kraichnan, On the Statistical Mechanics of an Adiabatically Compressible Fluid, J. Acoust. Soc. Am. 27, 438 (1955).

[52] L. S. G. Kovasznay, Turbulence in Supersonic Flow, J. Aeronaut. Sci. 20, 657 (1953).

[53] B.-T. Chu and L. S. G. Kovásznay, Non-linear Interactions in a Viscous Heat-Conducting Compressible Gas, J. Fluid Mech. 3, 494 (1958).

[54] S. Galtier and S. Banerjee, Exact Relation for Correlation Functions in Compressible Isothermal Turbulence, Phys. Rev. Lett. 107, 134501 (2011).

[55] S. Banerjee and S. Galtier, A Kolmogorov-like Exact Relation for Compressible Polytropic Turbulence, J. Fluid Mech. 742, 230 (2014).

[56] G. L. Eyink and T. D. Drivas, following paper, Cascades and Dissipative Anomalies in Relativistic Fluid Turbulence, following article, Phys. Rev. X 8, 011023 (2017).

[57] Y. Choquet-Bruhat, C. DeWitt-Morette, and M. DillardBleick, Analysis, Manifolds, and Physics, Part 1 (NorthHolland, Amsterdam, 1982).

[58] L. Onsager, Theories and Problems of Liquid Diffusion, Ann. N. Y. Acad. Sci. 46, 241 (1945).

[59] H. Brenner, Navier-Stokes Revisited, Physica (Amsterdam) 349A, 60 (2005).

[60] H. M. Mott-Smith, The Solution of the Boltzmann Equation for a Shock Wave, Phys. Rev. 82, 885 (1951). 
[61] H. W. Liepmann, R. Narasimha, and M. T. Chahine, Structure of a Plane Shock Layer, Phys. Fluids 5, 1313 (1962).

[62] E. Salomons and M. Mareschal, Usefulness of the Burnett Description of Strong Shock Waves, Phys. Rev. Lett. 69, 269 (1992).

[63] S.-H. Yu, Hydrodynamic Limits with Shock Waves of the Boltzmann Equation, Commun. Pure Appl. Math. 58, 409 (2005).

[64] V. G. Morozov, On the Langevin Formalism for Nonlinear and Nonequilibrium Hydrodynamic Fluctuations, Physica (Amsterdam) 126A, 443 (1984).

[65] G. L. Eyink, Dissipation and Large Thermodynamic Fluctuations, J. Stat. Phys. 61, 533 (1990).

[66] G. L. Eyink, Turbulence Noise, J. Stat. Phys. 83, 955 (1996).

[67] A. A. Mailybaev, Stochastic Anomaly and Large Reynolds Number Limit in Hydrodynamic Turbulence Models, arXiv:1508.03869.

[68] A. A. Mailybaev, Spontaneous Stochasticity of Velocity in Turbulence Models, Multiscale Model. Simul. 14, 96 (2016).

[69] A. A. Mailybaev, Spontaneously Stochastic Solutions in One-Dimensional Inviscid Systems, Nonlinearity 29, 2238 (2016).

[70] C. E. Leith and R. H. Kraichnan, Predictability of Turbulent Flows, J. Atmos. Sci. 29, 1041 (1972).

[71] D. Ruelle, Microscopic Fluctuations and Turbulence, Phys. Lett. A 72, 81 (1979).

[72] The limit appears to exist in an even stronger sense than distributionally, which implies limits of $Q_{\nu}$ exist only when smeared with $C^{\infty}$, compactly supported test functions. Experimentally, the limit seems to exist even if $Q_{\nu}$ is smeared with bounded continuous functions (weak convergence of measures) or even when integrated over compact sets, such as balls or cubes of fixed size as $\nu \rightarrow 0$. See Refs. [73,74].

[73] C. Meneveau and K. R. Sreenivasan, The Multifractal Spectrum of the Dissipation Field in Turbulent Flows, Nucl. Phys. B2, 49 (1987).

[74] C. Meneveau and K. R. Sreenivasan, The Multifractal Nature of Turbulent Energy Dissipation, J. Fluid Mech. 224, 429 (1991).

[75] S. Jagannathan and D. Donzis, High-Resolution Simulations of Forced Compressible Isotropic Turbulence, in American Physical Society, 64th Annual Meeting of the APS Division of Fluid Dynamics, 2011 (2011), http:// meetings.aps.org/link/BAPS.2011.DFD.E12.6.

[76] S. Jagannathan and D. A. Donzis, Reynolds and Mach Number Scaling in Solenoidally-Forced Compressible Turbulence Using High-Resolution Direct Numerical Simulations, J. Fluid Mech. 789, 669 (2016).

[77] Q. Ni, Y. Shi, and S. Chen, A Numerical Investigation on Active and Passive Scalars in Isotropic Compressible Turbulence, arXiv:1505.02685.

[78] S. R. De Groot and P. Mazur, Non-Equilibrium Thermodynamics, Dover Books on Physics (Dover, New York, 2013).

[79] L. D. Landau and E. M. Lifshitz, Fluid Mechanics, Course of Theoretical Physics, Translated from the Russian by
J. B. Sykes and W. H. Reid (Pergamon Press, New York, 1987), 2nd ed., Vol. 6.

[80] A. M. Obukhov, Temperature Field Structure in a Turbulent Flow, Izvestiia Akademii Nauk SSSR, Ser. Geogr. i Geofiz 13, 58 (1949).

[81] C. Normand, Y. Pomeau, and M. G. Velarde, Convective Instability: A Physicist's Approach, Rev. Mod. Phys. 49, 581 (1977).

[82] P. L. Lions, Mathematical Topics in Fluid Mechanics. Volume 1: Incompressible Models (Clarendon Press, Oxford, 1996).

[83] B. J. Bayly, C. D. Levermore, and T. Passot, Density Variations in Weakly Compressible Flows, Phys. Fluids A 4, 945 (1992).

[84] The assumption of small thermal expansion coefficients is true for most liquids, but for gases the temperature equation obtained by Ref. [83] contains another contribution from the advective derivative of the pressure.

[85] Here, we may note that the coarse-graining regularization also permits one to analyze dissipative anomalies in Lagrangian conservation laws such as fluid circulations [86] or magnetic fluxes [86] for advected loops, but it is unclear how to apply point splitting to Lagrangian invariants.

[86] G. L. Eyink, Turbulent Cascade of Circulations, C.R. Phys. 7, 449 (2006).

[87] L. P. Kadanoff, Scaling Laws for Ising Models near $T_{c}$, Physics 2, 263 (1966).

[88] K. G. Wilson, Renormalization Group and Critical Phenomena. I. Renormalization Group and the Kadanoff Scaling Picture, Phys. Rev. B 4, 3174 (1971).

[89] G. L. Eyink, Turbulent General Magnetic Reconnection, Astrophys. J. 807, 137 (2015).

[90] T. D. Drivas and G. L. Eyink, An Onsager Singularity Theorem for Turbulent Solutions of Compressible Euler Equations, Commun. Math. Phys., DOI: 10.1007/s00220017-3078-4 (2018).

[91] The failure to appreciate this point has been the source of many misunderstandings. For example, consider the following typical quote: "We therefore conclude that, for the large eddies which are the basis of any turbulent flow, the viscosity is unimportant and may be equated to zero, so that the motion of these eddies obeys Euler's equation. In particular, it follows from this that there is no appreciable dissipation of energy in the large eddies."-Landau and Lifshitz [92], Sec. 31. This statement is correct, if one understands it to mean that the viscous dissipation is negligible for the large eddies. However, the coarse graining that permits one to neglect viscosity at large scales generates new stresses that do not conserve the energy of the large eddies.

[92] L. D. Landau and E. M. Lifshitz, Fluid Mechanics, Course of Theoretical Physics, Translated from the Russian by J. B. Sykes and W. H. Reid, Vol. 6 (Pergamon Press, New York, 1959).

[93] The use of Favre-average cumulants rather than cumulants for the original spatial coarse graining is not essential. Their use does, however, reduce the number of additional cumulant terms that appear and permits a simple physical interpretation of each such term. For these reasons, Favre 
averaging has been popular in the practical engineering modeling of compressible turbulence [94].

[94] E. Garnier, N. Adams, and P. Sagaut, Large Eddy Simulation for Compressible Flows, Scientific Computation (Springer Netherlands, 2009).

[95] A. Favre, Statistical Equations of Turbulent Gases, in Problems of Hydrodynamics and Continuum Mechanics, edited by M. A. Lavrentiev (SIAM, Philadelphia, 1969), pp. 37-44.

[96] K. Huang, Statistical Mechanics (Wiley, New York, 1987).

[97] M. Germano, Turbulence: The Filtering Approach, J. Fluid Mech. 238, 325 (1992).

[98] One source of such stochasticity is thermal noise due to molecular degrees of freedom, so far neglected in our analysis, but other unknown perturbations can also lead to randomness. This stochasticity does not contradict our earlier claim of a "deterministic approach." A given weak Euler solution $\rho, \mathbf{v}, u$ over a particular time interval corresponds to a particular realization of these cumulants. It is only if one wants to predict or control the future behavior outside that particular time interval that one must recognize the intrinsic stochasticity. Here, we may note that vanishingly small stochastic perturbations do not alter our conclusions in Sec. IV regarding the description of coarse-grained variables in the inertial range by "weak Euler solutions." This argument may be made precise for thermal noise by appealing to the Onsager fluctuation principle $[65,99,100]$. The latter principle states that the probability of observing a particular set of fields $\rho, \mathbf{v}, u$ as thermal fluctuations is related to the additional dissipation or entropy production required to produce the fluctuation. See Ref. [65], Sec. IV, Example 2, for compressible NavierStokes fluids. Even when this excess dissipation is nonvanishing, the noise terms vanish in the coarse-grained equations for the limit $\eta, \zeta \kappa \rightarrow 0$. Details will be given elsewhere, but the argument is essentially the same as for the deterministic fluid equations in Sec. IV. Thus, coarsegrained variables in the inertial range are described by weak Euler solutions even in the presence of thermal noise.

[99] L. Onsager and S. Machlup, Fluctuations and Irreversible Processes, Phys. Rev. 91, 1505 (1953).

[100] R. Graham, Path-Integral Methods in Nonequilibrium Thermodynamics and Statistics, in Stochastic Processes in Nonequilibrium Systems (Springer, New York, 1978), pp. 82-138.

[101] C. Meneveau and J. Katz, Scale-Invariance and Turbulence Models for Large-Eddy Simulation, Annu. Rev. Fluid Mech. 32, 1 (2000).

[102] W. Schmidt, Large Eddy Simulations in Astrophysics, Living Rev. Comput. Astrophys. 1, 2 (2015).

[103] M. Oberguggenberger, Multiplication of Distributions and Applications to Partial Differential Equations, Pitman Research Notes in Mathematics Series, Vol. 259 (Longman Scientific \& Technical, Harlow, Essex, 1992).

[104] H. Aluie, S. Li, and H. Li, Conservative Cascade of Kinetic Energy in Compressible Turbulence, Astrophys. J. Lett. 751, L29 (2012).

[105] Q. Ni and S. Chen, Effects of Shock Topology on Temperature Field in Compressible Turbulence, arXiv:1506 .04179 .
[106] We caution that the Euler singularities required by Onsager's analysis need not be finite-time singularities arising spontaneously from smooth initial data but instead could be singularities input by solid boundaries or infinite-time singularities that develop in long-time steady states. For 3D compressible Euler equations, finite-time singularities of shock type have been proved to occur by other means [107].

[107] T. C. Sideris, Formation of Singularities in Three-Dimensional Compressible Fluids, Commun. Math. Phys. 101, 475 (1985).

[108] H. Aluie, Generalizing the 4/5-th Law to Compressible Turbulence (unpublished).

[109] G. L. Eyink, Turbulence Theory, Course Notes, http:// www.ams.jhu.edu/ eyink/Turbulence/notes.html.

[110] In fact, unpublished work of Aluie [108] shows how to recover the traditional 4/5th-law of Kolmogorov from such coarse-grained expressions for kinetic-energy flux.

[111] G. L. Eyink, Locality of Turbulent Cascades, Physica (Amsterdam) 207D, 91 (2005).

[112] A caveat has to do with the contribution of the density. If, as we have assumed in this work, the density is a bounded function and Hoelder continuous in space, then density increments $\delta \rho$ are scale local. However, the coarse-grained density $\bar{\rho}$ in that case is dominated by energy-scale contributions, and infrared locality breaks down. On the other hand, there is evidence from numerical simulations that, for Mach numbers much larger than 1, the density in compressible fluid turbulence exists only as a distribution (measure) in the infinite Reynolds-number limit [113]. In that case, $\bar{\rho}$ is scale local; however, $\delta \rho$ is dominated by dissipation-range contributions, and ultraviolet-locality breaks down. In either case, scale locality through the dependence on density is always broken in one direction.

[113] J. Kim and D. Ryu, Density Power Spectrum of Compressible Hydrodynamic Turbulent Flows, Astrophys. J. Lett. 630, L45 (2005).

[114] R. Becker, Stosswelle und Detonation, Z. Phys. 8, 321 (1922) [Impact Waves and Detonation, NACA Tech. Memo. NACA-TM-505 and NACA-TM-506 (1929)].

[115] B. M. Johnson, Analytical Shock Solutions at Large and Small Prandtl Number, J. Fluid Mech. 726, R4 (2013).

[116] The notion of energy cascade has sometimes been criticized as unphysical because it depends upon arbitrary scale decompositions. For example, consider the following quotes from one prominent critic: "On the other hand, energy transfer, just like any physical process, should be invariant of particular decompositions/representations of a turbulent field. In this sense Kolmogorov's choice of dissipation (and energy input) are well defined and decomposition independent quantities, whereas the energy flux is (generally) not, since it is decomposition dependent. After all Nature may and likely does not know about our decompositions."-Tsinober [117], and "We have seen that there is an ambiguity in defining the meaning of the term 'small scales' (or more generally 'scales' or 'eddies', see appendix C) and consequently the meaning of the term 'cascade'."-Tsinober [117]. One erroneous statement above is the claim that energy flux is 
"generally" decomposition dependent. In fact, the energy cascade rate over a long inertial range at high Reynolds numbers is demonstrably the same for any filter kernel satisfying very general, mild assumptions of smoothness and rapid spatial decay. However, the other remarks are correct and acute. Indeed, the physical process must be invariant of particular decompositions or representations and independent of the scale of observation. What the criticism is missing is that the requirement of such invariance is a positive principle that can be exploited to deduce exact consequences.

[117] A. Tsinober, An Informal Conceptual Introduction to Turbulence: Second Edition of An Informal Introduction to Turbulence, Fluid Mechanics and Its Applications (Springer, Netherlands, 2009).

[118] A. N. Kolmogorov, A Refinement of Previous Hypotheses Concerning the Local Structure of Turbulence in a Viscous Incompressible Fluid at High Reynolds Number, J. Fluid Mech. 13, 82 (1962).

[119] R. H. Kraichnan, On Kolmogorov's Inertial-Range Theories, J. Fluid Mech. 62, 305 (1974).

[120] At least the results are distinct for $\operatorname{Pr}=0, \operatorname{Pr}=\infty$, and $0<\operatorname{Pr}<\infty$. We have analytical results for only one finite positive value $\operatorname{Pr}=3 / 4$.

[121] J. P. Peixoto, A. H. Oort, M. De Almeida, and A. Tomé, Entropy Budget of the Atmosphere, J. Geophys. Res. Atmos. 96, 10981 (1991).

[122] O. Pauluis and I. M. Held, Entropy Budget of an Atmosphere in Radiative-Convective Equilibrium. Part I: Maximum Work and Frictional Dissipation, J. Atmos. Sci. 59, 125 (2002).

[123] S. I. Braginskii, Transport Processes in a Plasma, in Reviews of Plasma Physics, Authorized translation from the Russian by Herbert Lashinsky, University of Maryland, edited by M. A. Leontovich (Consultants Bureau, New York, 1965), Vol. 1, pp. 205-311.

[124] A. A. Schekochihin, S. C. Cowley, W. Dorland, G. W. Hammett, G. G. Howes, G. G. Plunk, E. Quataert, and T Tatsuno, Gyrokinetic Turbulence: A Nonlinear Route to Dissipation through Phase Space, Plasma Phys. Controlled Fusion 50, 124024 (2008).

[125] A. A. Schekochihin, S. C. Cowley, W. Dorland, G. W. Hammett, G. G. Howes, E. Quataert, and T. Tatsuno, Astrophysical Gyrokinetics: Kinetic and Fluid Turbulent Cascades in Magnetized Weakly Collisional Plasmas, Astrophys. J. Suppl. Ser. 182, 310 (2009).

[126] D. Ruelle, Statistical Mechanics: Rigorous Results (World Scientific, Singapore, 1999).

[127] A. Martin-Löf, Statistical Mechanics and the Foundations of Thermodynamics, Lecture Notes in Physics (SpringerVerlag, Berlin, 1979).

[128] H. B. Callen, Thermodynamics and an Introduction to Thermostatistics (Wiley, New York, 1985).

[129] J. W. Gibbs, A Method of Geometrical Representation of the Thermodynamic Properties of Substances by Means of Surfaces, Trans. Conn. Acad. Arts Sci. 2, 382 (1873); Reprinted in: The Collected Works of J. Willard Gibbs, Volume I. Thermodynamics (Yale University Press, New Haven, 1948), pp. 33-54.
[130] L. Brillouin, The Negentropy Principle of Information, J. Appl. Phys. 24, 1152 (1953).

[131] Here, the letter " $I$ " may stand either for "input" or for "information," which is another synonym for negentropy.

[132] For completeness, we note here, without proof, the relevant identity. Let $f(\rho)$ be any smooth function of a set of density functions $\rho=\left(\rho_{1}, \ldots, \rho_{m}\right)$, and let $\rho(\mathbf{x})$ be a spatial field of the densities. Then, $\Delta f=\overline{f(\rho)}-f(\bar{\rho})$ is equal to $\int d^{d} r G_{\ell}(\mathbf{r})[f(\rho(\mathbf{x}+\mathbf{r}))-f(\rho(\mathbf{x}))-\delta \rho(\mathbf{x} ; \mathbf{r}) \cdot(\nabla f)(\rho(\mathbf{x}))]$ minus $f(\bar{\rho}(\mathbf{x}))-f(\rho(\mathbf{x}))+\rho^{\prime}(\mathbf{x}) \cdot(\boldsymbol{\nabla} f)(\rho(\mathbf{x}))$, defining $\delta \rho(\mathbf{x} ; \mathbf{r})=\rho(\mathbf{x}+\mathbf{r})-\rho(\mathbf{x}), \rho^{\prime}(\mathbf{x})=\rho(\mathbf{x})-\bar{\rho}(\mathbf{x})$.

[133] We use the terms "inverse cascade of entropy" and "forward cascade of negentropy" essentially interchangeably. Note that thermodynamic entropy can be made arbitrarily large by heating and arbitrarily small by cooling (unless temperatures drop to a range near absolute zero, where quantum effects provide a lower bound to entropy). Regardless of the initial entropy content at any length scale, large-scale cooling can input negentropy, and small-scale molecular transport may produce positive entropy. Thus, either may be regarded as the "source" and the other as the "sink."

[134] We are not being entirely consistent with our mathematical notations. We should have defined $\beta \circ Q_{\text {visc }}=$ $\lim _{\ell \rightarrow 0} \bar{\beta} \cdot \bar{Q}_{\text {visc }}$, and the quantities in Eqs. (85) and (86) would require a new notation. To avoid a proliferation of new symbols, we use the same notation "o" everywhere for the relevant distributional products obtained by limits of $\ell$ through the inertial range, whereas the notation "*" stands for the distributional product obtained by limits $\eta, \zeta, \kappa \rightarrow 0$ of fine-grained or dissipation-range quantities.

[135] Because of the assumed smoothness of $Q_{\text {cool }}$, the product $\beta Q_{\text {cool }}$ is meaningful in the ordinary sense in the ideal limit, and no special care is required for its definition.

[136] Obukhov implicitly assumed that mechanical forcing is negligible compared to thermal forcing, with the latter due to either large initial temperature inhomogeneities or to a strong external source of heating or cooling. Under that assumption, the entropy production (22) arises predominantly from the thermal conduction term $\Sigma_{\text {therm, }}$, and the viscous heating contributions $\beta Q_{\text {visc }}$ can be neglected as relatively small. In a statistical steady state for the regime considered by Obukhov, the entropy balance (106) becomes $\left\langle\beta Q_{\text {cool }}\right\rangle=\left\langle\Sigma_{\text {therm }}\right\rangle$.

[137] A. M. Yaglom, On the Local Structure of a Temperature Field in a Turbulent Flow, Dokl. Akad. Nauk SSSR 69, 743 (1949).

[138] G. Erlebacher, M. Y. Hussaini, H. O. Kreiss, and S. Sarkar, The Analysis and Simulation of Compressible Turbulence, Theor. Comput. Fluid Dyn. 2, 73 (1990).

[139] A. G. Kritsuk, M. L. Norman, P. Padoan, and R. Wagner, The Statistics of Supersonic Isothermal Turbulence, Astrophys. J. 665, 416 (2007).

[140] A. G. Kritsuk, R. Wagner, and M. L. Norman, Energy Cascade and Scaling in Supersonic Isothermal Turbulence, J. Fluid Mech. 729, R1 (2013).

[141] S. Chapman and T. G. Cowling, The Mathematical Theory of Non-uniform Gases (Cambridge University Press, Cambridge, England, 1970). 
[142] J. A. McLennan, Introduction to Nonequilibrium Statistical Mechanics (Prentice-Hall, Englewood Cliffs, NJ, 1989).

[143] D. N. Zubarev, Nonequilibrium Statistical Thermodynamics (Consultants Bureau, New York, 1974).

[144] This problem was acknowledged in some of the early works assuming isentropy. For example, Chandrasekhar [50] admitted that "It is arguable that the assumption of the adiabatic relation ... is not compatible with the retention of the term in viscosity...."

[145] D. A. Donzis and S. Jagannathan, Fluctuations of Thermodynamic Variables in Stationary Compressible Turbulence, J. Fluid Mech. 733, 221 (2013).

[146] R. Narayan and M. V. Medvedev, Thermal Conduction in Clusters of Galaxies, Astrophys. J. Lett. 562, L129 (2001).

[147] A. Lazarian, Enhancement and Suppression of Heat Transfer by MHD Turbulence, Astrophys. J. Lett. 645, L25 (2006).

[148] N. N. Bogoliubov and D. V. Shirkov, Quantum Fields (Benjamin-Cummings, Reading, MA, 1983).

[149] E. Feireisl, P. Gwiazda, A. Świerczewska-Gwiazda, and E. Wiedemann, Regularity and Energy Conservation for the Compressible Euler Equations, Arch. Ration. Mech. Anal. 223, 1375 (2017).

[150] R. Wagner, G. Falkovich, A. G. Kritsuk, and M. L. Norman, Flux Correlations in Supersonic Isothermal Turbulence, J. Fluid Mech. 713, 482 (2012).

[151] G. L. Eyink and H. Aluie, The Breakdown of Alfvén's Theorem in Ideal Plasma Flows: Necessary Conditions and Physical Conjectures, Physica (Amsterdam) 223D, 82 (2006).

[152] For finite values of $\eta, \zeta, \kappa$, the modes have complex frequencies with imaginary parts reflecting dissipative decay. For example, see Ref. [53], Eqs. 6.1-3. Here, we focus on the ideal nonlinear behavior at very high Reynolds and Péclet numbers, as in most of Ref. [53], Sec. VI.

[153] Here, we note that the argument of Ref. [83], that the entropy fluctuation $s_{n}^{\prime}$ becomes a passive scalar in the limit of small Mach number, is fallacious. Considering their entropy balance equation (15) in the low-Ma regime, the authors of Ref. [83] claimed that "in the inertial range, the right side of (15) is negligible." This statement is erroneous and ignores the fact that kinetic-energy dissipation according to their limiting incompressible velocity equation (8b) is observed in experiments such as Refs. [28-30] to be nonvanishing even in the limit as $\operatorname{Re} \rightarrow \infty$.

[154] B. Tao, J. Katz, and C. Meneveau, Statistical Geometry of Subgrid-Scale Stresses Determined from Holographic Particle Image Velocimetry Measurements, J. Fluid Mech. 457, 35 (2002).

[155] The most serious errors in the results of Ref. [105] are in their Eqs. (6.6)-(6.8), which correspond to the last three terms on the right-hand side of our Eq. (144). In particular, the pressure work $\overline{p \Theta}$ in their Eq. (6.6) is factorized as $\bar{p} \bar{\Theta}$ and, most seriously, $\bar{Q}_{\text {visc }}$ in their Eq. (6.7) is replaced with $2 \eta|\overline{\mathbf{S}}|^{2}+\zeta|\bar{\Theta}|^{2}$. This latter quantity vanishes in the ideal limit of high Reynolds numbers, whereas the quantity $\bar{Q}_{\text {visc }}$ is expected to have a nonzero value.
[156] Our rigorous inequalities for structure-function exponents are all valid for orders $q \geq 3$, where the values must be sub-Kolmogorov. As usual, the exponents for $q \leq 3$ are then expected to have super-Kolmogorov values because of the concavity of the scaling exponents in the variable $q$ [37].

[157] J. W. Armstrong, J. M. Cordes, and B. J. Rickett, Density Power Spectrum in the Local Interstellar Medium, Nature (London) 291, 561 (1981).

[158] J. W. Armstrong, B. J. Rickett, and S. R. Spangler, Electron Density Power Spectrum in the Local Interstellar Medium, Astrophys. J. 443, 209 (1995).

[159] T. J. W. Lazio, J. M. Cordes, A. G. De Bruyn, and J.-P. Macquart, The Microarcsecond Sky and Cosmic Turbulence, New Astron. Rev. 48, 1439 (2004).

[160] A. Chepurnov and A. Lazarian, Extending the Big Power Law in the Sky with Turbulence Spectra from Wisconsin Ho Mapper Data, Astrophys. J. 710, 853 (2010).

[161] L. D. Landau, J. S. Bell, M. J. Kearsley, L. P. Pitaevskii, E. M. Lifshitz, and J.B. Sykes, Electrodynamics of Continuous Media, Course of Theoretical Physics, Vol. 8 (Elsevier Science, New York, 2013).

[162] H. Aluie and G. L. Eyink, Scale Locality of Magnetohydrodynamic Turbulence, Phys. Rev. Lett. 104, 081101 (2010).

[163] R. M. Kulsrud, Plasma Physics for Astrophysics, Princeton Series in Astrophysics (Princeton University Press, Princeton, NJ, 2005).

[164] J. C. Higdon, Density Fluctuations in the Interstellar Medium: Evidence for Anisotropic Magnetogasdynamic Turbulence. I-Model and Astrophysical Sites, Astrophys. J. 285, 109 (1984).

[165] Y. Lithwick and P. Goldreich, Compressible Magnetohydrodynamic Turbulence in Interstellar Plasmas, Astrophys. J. 562, 279 (2001).

[166] To make this argument correctly requires a discussion of the linear wave modes of compressible MHD. This problem is carefully treated by Ref. [165], Appendix A, including also the important effects of cooling. As discussed by those authors, the MHD entropy mode with cooling has, at sufficiently small scales, zero perturbation not of thermal pressure but of total pressure (thermal + magnetic). Nevertheless, their analysis shows that the above argument based upon the entropy mode of a hydrodynamic (nonmagnetized) fluid carries over to MHD with only minor changes. In fact, the hydrodynamic treatment of the entropy mode is exactly valid at sufficiently small scales where slow magnetosonic waves can create pressure balance of the entropy mode so that $p^{\prime}=0$. At larger scales, the entropy modes carries a nonvanishing fluctuation $p^{\prime} \neq 0$ of thermal pressure, which is balanced by a magnetic pressure fluctuation. In either case, the entropy mode is rapidly damped on a cooling time scale $t_{\text {cool }}$, so the amplitude of the entropy mode can be argued to be small above a cooling scale $L_{\text {cool }}$. See Ref. [165], Appendix A, for all details.

[167] One example of a failure of the Kovásznay mode-coupling theory which has already been considered is its prediction that the entropy per particle $s_{n}^{\prime}$ is a passive scalar. As another example, consider the estimate by Ref. [165], 
Sec. V.2, for the spectrum of density at $\mathrm{Ma}=1$ in a regime ("high- $\beta$ ") where magnetic pressure is small relative to thermal pressure. Invoking Kovásznay's quadratic soundsound coupling $\partial^{2}\left(v_{i}^{\prime} v_{j}^{\prime}\right) / \partial x_{i} \partial x_{j}$, they predicted that density perturbations due to isothermal sound waves will lead to a $k^{-7 / 3}$ density spectrum. Instead, Ref. [168], in a simulation of isothermal compressible MHD at Ma $\sim 1$, observed, for large $\beta$, a $k^{-5 / 3}$ density spectrum, and Ref. [113], for isothermal hydrodynamic $(\beta=\infty)$ turbulence at $\mathrm{Ma} \sim 1$, also found a $k^{-5 / 3}$ density spectrum, contradicting mode-coupling predictions.

[168] G. Kowal, A. Lazarian, and A. Beresnyak, Density Fluctuations in MHD Turbulence: Spectra, Intermittency, and Topology, Astrophys. J. 658, 423 (2007).

[169] B. M. Johnson, Closed-Form Shock Solutions, J. Fluid Mech. 745, R1 (2014).

[170] Our notation differs from that of Johnson [115,169], with our $\eta$ equal to Johnson's $(4 / 3) \mu$. 